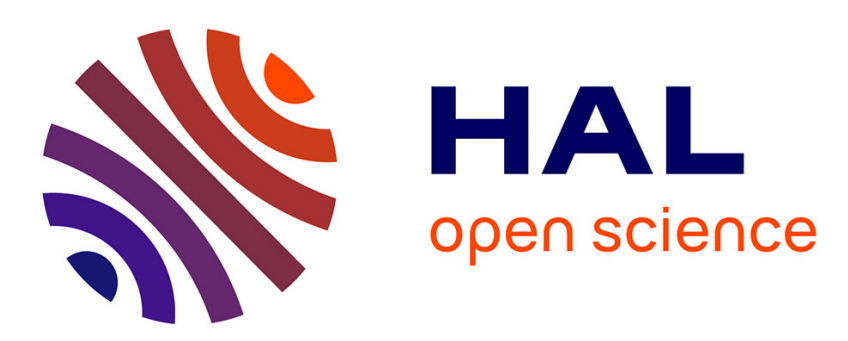

\title{
Reliability-oriented sensitivity analysis in presence of data-driven epistemic uncertainty
}

Gabriel Sarazin, Jérôme Morio, Agnès Lagnoux, Mathieu Balesdent, Loïc Brevault

\section{- To cite this version:}

Gabriel Sarazin, Jérôme Morio, Agnès Lagnoux, Mathieu Balesdent, Loïc Brevault. Reliabilityoriented sensitivity analysis in presence of data-driven epistemic uncertainty. Reliability Engineering and System Safety, 2021, 215, pp.107733. 10.1016/j.ress.2021.107733 . hal-03461603

\section{HAL Id: hal-03461603 \\ https://hal.science/hal-03461603}

Submitted on 1 Dec 2021

HAL is a multi-disciplinary open access archive for the deposit and dissemination of scientific research documents, whether they are published or not. The documents may come from teaching and research institutions in France or abroad, or from public or private research centers.
L'archive ouverte pluridisciplinaire HAL, est destinée au dépôt et à la diffusion de documents scientifiques de niveau recherche, publiés ou non, émanant des établissements d'enseignement et de recherche français ou étrangers, des laboratoires publics ou privés. 


\title{
Reliability-oriented sensitivity analysis in presence of data-driven epistemic uncertainty
}

\author{
Gabriel Sarazin ${ }^{\mathrm{a}, *}$, Jérôme Morio $^{\mathrm{a}}$, Agnès Lagnoux ${ }^{\mathrm{b}}$, Mathieu Balesdent ${ }^{\mathrm{c}}$, Loïc Brevault ${ }^{\mathrm{c}}$ \\ ${ }^{a}$ ONERA/DTIS, Université de Toulouse, Toulouse, France \\ ${ }^{b} I M T$, Université de Toulouse Jean Jaurès, Toulouse, France \\ ${ }^{c}$ ONERA/DTIS, Université de Paris-Saclay, Palaiseau, France
}

\begin{abstract}
Reliability assessment in presence of epistemic uncertainty leads to consider the failure probability as a quantity depending on the state of knowledge about uncertain input parameters. The input joint distribution is often learnt from a small-sized dataset provided by operating experience. The computed failure probability depends on the estimated marginal distributions and the estimated copula distribution. This paper develops a reliability-oriented sensitivity analysis procedure in order to measure the influence exerted by the data-driven modeling of both the margins and the copula. The proposed methodology is validated for both deterministic and stochastic reliability methods through an extensive simulation study including several analytical performance functions as well as a real-life simulation code dealing with the buckling of a composite laminate plate.
\end{abstract}

Keywords: Sensitivity analysis, rare-event simulation, dependence modeling, data-driven modeling.

\section{Introduction}

Reliability assessment often requires computing a failure probability by coupling a highly expensive simulation code and some rare-event probability estimation techniques [1, 2]. The characterization of the input joint distribution is essential in reliability assessment and must incorporate appropriate descriptions of the tails and the dependence structure [3, 4]. In presence of dependent inputs, the copula-marginal decomposition offers a convenient framework where the dependence structure is isolated from marginal trends. When the input joint distribution is known in advance, the failure probability estimation is subject to variability because of the noise introduced by the reliability method.

In many cases, there is also a lack of knowledge about the input joint distribution and this gives birth to epistemic uncertainties [5]. Because of the simultaneous impact of aleatory and epistemic uncertainties, the failure probability is said to be affected by mixed uncertainties. In the literature, the second level of uncertainty is often modeled with probability theory. Nevertheless, beyond the

\footnotetext{
*Corresponding author: Gabriel.Sarazin@onera.fr
} 
well-established probability theory, a more general framework consists in characterizing uncertain model parameters with imprecise probabilities. This encompasses notably Dempster-Shafer's theory of evidence [6, 7], possibility theory [8, 9], probability-boxes [10, 11], Bayesian hierarchical models [12, 13], random sets $[14,15]$ as well as fuzzy sets [16, 17] and measures [18]. Only a few articles propose to extend rare-event simulation techniques [19] to the formalism of imprecise probabilities. The main difficulty is the required simulation budget. All research works on this topic endeavor to reduce the computational cost induced by a Monte Carlo-based propagation of imprecise probabilities [20]. Subset simulation and random sets are coupled in [21] while the FORM/SORM methodology is combined with evidence theory in [22]. Line sampling is adapted to imprecise probabilities in [23]. The joint use of p-boxes and surrogate models is described in [24]. In sensitivity analysis (SA), dealing with imprecise probabilities is not much developed in the literature because it implies a significant computational load. For instance, variance-based SA has been performed with evidence theory [25], Bayesian hierarchical models [26] and random sets [27]. SA can also be achieved in presence of p-boxes provided that a surrogate model is set up to replace the simulation code [28]. The confluence of reliability assessment and SA is called reliability-oriented sensitivity analysis (ROSA). It ambitions to identify the parameter that conveys most uncertainty during reliability assessment [29, 30].

The main interest of this work consists in performing global ROSA with respect to the margins and the copula when the joint distribution is unknown and needs to be learnt from a small amount of data. As this situation is very common is practice, uncertainty management in presence of insufficient data is gaining increasing interest in the literature [31, 32, 33]. The question here is to identify which component of the estimated joint distribution (either the estimated margins or the estimated copula) is responsible for the largest share of uncertainty. If a marginal distribution is emerging, collecting additional data for the related input variable would be of great help in order to mitigate the uncertainty due to the estimation of this component. The ROSA procedure which is devised in this work is entirely data-driven and the starting material only consists of one single dataset. The resulting workflow will be shown to work properly provided that the sample size does not become excessively small. Furthermore, computing a failure probability from one given input distribution is cumbersome from a computational viewpoint and cannot be repeated too many times. As a result, the ROSA algorithm will be adapted to the simulation budget.

The remainder of this paper is organized as follows. In Section 2, the ROSA background is described in details. In Section 3, under the assumption of negligible computational costs and exact reliability assessment, a first version of the ROSA algorithm is come up with. In Section 4, it is explained how to upgrade the initial ROSA algorithm so that its enhanced version can meet situations where the preceding assumptions are relaxed. In order to achieve this, it is shown that ROSA can take advantage of various existing computational strategies that allow for better feasibility [34] and stronger 
robustness [35]. The complete methodology is then applied to the reliability assessment of a composite laminate plate where the joint distribution of bending stiffness coefficients is learnt with kernel density estimation (KDE) and regular vine copulas (R-vines).

\section{Reliability assessment in presence of epistemic uncertainties}

\subsection{Probabilistic reliability assessment}

In many industrial field, reliability analysis often boils down to examining how a variable of interest behaves with respect to a predefined failure criterion. This quantity is computed from an expensive simulation code $\phi(\cdot)$ which is called the performance function. A black-box approach is often adopted to represent the input-output relationship:

$$
\begin{aligned}
& \phi: \quad \mathbb{R}^{d} \quad \longrightarrow \quad \mathbb{R} \\
& \boldsymbol{x}=\left(x_{1}, \ldots, x_{d}\right) \longmapsto y=\phi(\boldsymbol{x})
\end{aligned}
$$

Input quantities are often stochastic, namely subject to a natural form of variability which is inherent to the physical system or to the environment under consideration [36]. In the literature, such uncertainties are often referred to as aleatory [37] and are non-reducible by adding knowledge. As a result of uncertainty propagation, the output variable is random as well. Various mathematical structures were developed to represent input and output uncertainties but the present work is only based on the standard probabilistic framework [38]. The joint probability density function (PDF) and joint cumulative distribution function (CDF) of the input random vector $\boldsymbol{X}=\left[X_{1}, \ldots, X_{d}\right]$ are respectively denoted by $f_{\boldsymbol{X}}$ and $F_{\boldsymbol{X}}$. Likewise, let $f_{X_{j}}$ and $F_{X_{j}}$ be the marginal PDF and CDF characterizing the distribution of each random variable $X_{j}$. The output random variable $Y=\phi(\boldsymbol{X})$ is entirely then characterized by the pushforward density $f_{Y}$ spawned by $f_{\boldsymbol{X}}$ after propagation through $\phi$. In this work, the unwanted event is $\{Y>T\}$ and the associated failure domain is denoted by $D_{f}$. The failure probability $P_{f}$ is thus defined by:

$$
\begin{aligned}
P_{f} & =\mathbb{P}\{Y>T\}=\int_{T}^{+\infty} f_{Y}(y) \mathrm{d} y \\
& =\mathbb{E}\left[\mathbb{1}_{D_{f}}(\boldsymbol{X})\right]=\mathbb{P}\left\{\boldsymbol{X} \in D_{f}\right\}=\int_{\mathbb{R}^{d}} \mathbb{1}_{D_{f}}(\boldsymbol{x}) f_{\boldsymbol{X}}(\boldsymbol{x}) \mathbf{d} \boldsymbol{x} \\
& :=\mathcal{R}\left(f_{\boldsymbol{X}}\right) .
\end{aligned}
$$

$\mathcal{R}$ stands for mathematical integration over the failure domain $D_{f}$ for any given $\operatorname{PDF} f_{\boldsymbol{X}} \in \mathbb{L}^{2}\left(\mathbb{R}^{d}\right)$. In most practical situations, $\mathcal{R}$ is unachievable and the exact value of $P_{f}$ cannot be evaluated. Monte Carlo simulations are often the only workable way of estimating $P_{f}$. If so, reliability assessment is no

longer deterministic. As this method depends on the drawn samples, each estimate $\hat{P}_{f}$ of the failure 
probability is one realization of the crude Monte Carlo (CMC) algorithm defined by:

$$
\hat{P}_{f}=\frac{1}{N_{s}} \sum_{i=1}^{N_{s}} \mathbb{1}_{\left\{Y^{(i)}>T\right\}} \quad \text { with }\left\{\begin{array}{l}
\boldsymbol{X}^{(i)} \stackrel{\text { i.i.d }}{\sim} f_{\boldsymbol{X}} \\
Y^{(i)}=\phi\left(\boldsymbol{X}^{(i)}\right)
\end{array}\right.
$$

When $P_{f}$ becomes a rare-event probability, too many samples are needed by CMC to reach a sufficient level of accuracy. Rare event simulation $[2,19]$ includes a wide range of sampling techniques in order to find a trade-off between variance reduction and computational viability. Whatever is the nondeterministic method used to estimate $P_{f}$, the resulting estimator may be expressed as:

$$
\hat{P}_{f}:=\mathcal{R}_{w}\left(f_{\boldsymbol{X}}\right),
$$

where $w$ is the randomness introduced by the sampling process. For instance, $w$ stands for the random generation of input samples $\boldsymbol{X}^{(i)}$ in Eq. (5). $\mathcal{R}_{w}$ may be considered as a stochastic approximation of $\mathcal{R}$ relying on Monte Carlo-like experiments. From now on, the uncertainty surrounding how $w$ behaves is called forecast uncertainty.

\subsection{Reliability assessment with dependent inputs}

In many application fields including hydrology [39, 40], geostatistics [41, 42], finance [43, 44] and insurance $[45,46]$, it is now generally agreed that the joint $\operatorname{PDF} f_{\boldsymbol{X}}$ has to take into account the statistical dependence structure existing among the variables under study. Without such a precaution, $P_{f}$ may be seriously underestimated or overestimated. The copula-marginal separation (enunciated by Sklar's theorem [47]) is a convenient way to deal with dependence modeling. The joint CDF $F_{\boldsymbol{X}}$ may be expressed as follows:

$$
F_{\boldsymbol{X}}(\boldsymbol{x})=F_{\boldsymbol{X}}\left(x_{1}, \ldots, x_{d}\right)=C_{\boldsymbol{U}}\left(F_{X_{1}}\left(x_{1}\right), \ldots, F_{X_{d}}\left(x_{d}\right)\right),
$$

where $C_{\boldsymbol{U}}$ is called the copula CDF because it is nothing but the CDF of the random vector gathering all probability-transformed variables:

$$
\boldsymbol{U}=\left[U_{1}, \ldots, U_{d}\right]=\left[F_{X_{1}}\left(X_{1}\right), \ldots, F_{X_{d}}\left(X_{d}\right)\right] .
$$

Since all variables $U_{j}$ are uniformly distributed, the actual support of $\boldsymbol{U}$ is the hypercube $\mathcal{H}_{d}=[0,1]^{d}$. Differentiation applied on Eq. (7) provides the following expression of the joint PDF:

$$
f_{\boldsymbol{X}}(\boldsymbol{x})=f_{\boldsymbol{X}}\left(x_{1}, \ldots, x_{d}\right)=\underbrace{c_{\boldsymbol{U}}\left(F_{X_{1}}\left(x_{1}\right), \ldots, F_{X_{d}}\left(x_{d}\right)\right)}_{c_{\boldsymbol{U}}(\boldsymbol{u})} \times\left[\prod_{j=1}^{d} f_{X_{j}}\left(x_{j}\right)\right],
$$

where the copula PDF $c_{\boldsymbol{U}}$ is the cross partial derivative of $C_{\boldsymbol{U}}$. In the right-hand side of Eq. (9), the copula term $c_{U}(\boldsymbol{u})$ that goes with the well-known product of marginal PDFs must be seen as an additional factor which accounts for the dependence structure. For further details, the interested reader may consult $[48,49]$. 


\subsection{Reliability assessment under imperfect state of knowledge}

One of the cornerstones of reliability analysis is the input PDF $f_{\boldsymbol{X}}$ characterizing how operational variables are inter-related. In many situations, engineering expertise is neither available nor sufficiently trustworthy to decide upon a specification which is likely to receive unanimous expert agreement. The lack of knowledge about $f_{\boldsymbol{X}}$ is an ubiquitous and challenging problem for practitioners because reliability analysis can no longer rest on the computation of one single failure probability. This second level of uncertainty, which encompasses all the factors leading to envision $f_{\boldsymbol{X}}$ as a random entity, is termed epistemic. Distinguishing between aleatory and epistemic uncertainties has always been of great interest in uncertainty analysis since it allows the analyst to identify the uncertainty sources that might potentially be reduced with updated knowledge or enhanced models [50, 51].

\subsubsection{The Bayesian approach}

One possible framework to incorporate epistemic uncertainties consists in adopting the Bayesian point of view [13]. The input PDF parameters are assumed unknown and represented by a random vector $\Theta=\left[\Theta_{1}, \ldots, \Theta_{p}\right]$. The distribution of $\Theta$ can be chosen based on prior knowledge (a prior $\operatorname{PDF} f_{\boldsymbol{\Theta}}(\cdot \mid \boldsymbol{\xi})$ with support $D_{\boldsymbol{\theta}}$ and hyperparameters $\left.\boldsymbol{\xi}\right)$ or it can be inferred from available field data through Bayesian analysis [52]. Then, one can easily adapt Eq. (4) and (6) as follows:

$$
\begin{aligned}
& P_{f}(\boldsymbol{\Theta})=\mathcal{R}\left(f_{\boldsymbol{X} \mid \boldsymbol{\Theta}}\right)=\int_{\mathbb{R}^{d}} \mathbb{1}_{D_{f}}(\boldsymbol{x}) f_{\boldsymbol{X} \mid \boldsymbol{\Theta}}(\boldsymbol{x}) \mathbf{d} \boldsymbol{x} \\
& \hat{P}_{f}(\boldsymbol{\Theta})=\mathcal{R}_{w}\left(f_{\boldsymbol{X} \mid \boldsymbol{\Theta}}\right) .
\end{aligned}
$$

One can see that the resulting failure probabilities directly depend on $\Theta$. In Eq. (11), the additional uncertainty due to $\Theta$ is supposed to outweigh the uncertainty introduced by the use of the CMC algorithm. Specific case studies in engineering applications are proposed in [53, 54]. In presence of this bi-level input uncertainty, an averaged reliability measure is defined in [55] by:

$$
\widetilde{P}_{f}(\boldsymbol{\xi})=\mathbb{E}\left[P_{f}(\boldsymbol{\Theta})\right]=\int_{\mathbb{R}^{d} \times D_{\boldsymbol{\theta}}} \mathbb{1}_{D_{f}}(\boldsymbol{x}) f_{\boldsymbol{X} \mid \boldsymbol{\Theta}}(\boldsymbol{x} \mid \boldsymbol{\theta}) f_{\boldsymbol{\Theta}}(\boldsymbol{\theta} \mid \boldsymbol{\xi}) \mathrm{d} \boldsymbol{\theta} \mathrm{d} \boldsymbol{x}
$$

This expression suggests to estimate $\tilde{P}_{f}(\boldsymbol{\xi})$ with a double-loop Monte Carlo algorithm involving a failure probability computation (inner loop) for each value taken by $\Theta$ (outer loop). As highlighted in [56], such a nested procedure is intuitive but it is outperformed by the augmented reliability approach described in $[57,58]$. It must be said that the vast majority of papers on this topic assume that input variables $X_{j}$ are mutually independent. As it is often vain to look for trusted information about the copula model, copula-based multivariate distributions do not easily fit into Bayesian reliability analysis. The epistemic uncertainty conveyed by copula estimation is discussed in the next subsection. 


\subsubsection{The lack of knowledge about the copula}

In many works investigating how the lack of knowledge about the copula PDF may bear on reliability analysis, the marginal distributions are assumed to be prescribed. The functional space of all possible distributions is thus restricted to a Fréchet class [59]. Popular methods to fit a copula distribution include the maximization of an information criterion [60], graphical selection procedures [61, 62], goodness-of-fit tests [63, 64] or Bayesian selection [65, 66]. Moreover, the need for appropriate models to tackle high-dimension dependence structures has motivated the development of more flexible copula classes such as regular vine copulas [67], hierarchical Archimedean copulas [68], copula Bayesian networks [69], factor copula models [70] and products of bivariate copulas [71]. They are all based on graph representations that ambition to sketch the backbone of the dependence structure. This approach has allowed for major advances in order to (a) create efficient associations of variables, (b) establish a hierarchy among dependence substructures and (c) introduce parsimonious specifications in high dimension.

Sometimes, the correlation coefficients are also known in advance. If so, the state of knowledge about the input distribution is a predefined set of margins and covariances. Some multivariate distributions $[72,73]$ are tailored to be consistent with this kind of prior knowledge. This problem is also adressed in [74] where an iterative algorithm is used to calibrate a vine copula distribution under the constraint of a prescribed correlation matrix. The common denominator of these works is the lack of knowledge about the copula type. When correlations are provided, uncertainty on the copula distribution is limited to model selection. It is however an issue of utmost importance since it is ascertained in [3] that the failure probabilities computed from identically correlated copulas may differ considerably. In response, an algorithm to find the worst-case vine copula in terms of reliability assessment was proposed in [75].

\subsubsection{Copula-based descriptions of epistemic uncertainties}

In the general case where the marginal distributions and the copula distribution have to be learnt, there are very few works that propagate epistemic uncertainties with a copula-focused approach. The notion of "imprecise copula" [76] is an extension of Sklar's theorem in order to consider a set of copulas instead of a single one. This mathematical concept is a notable breakthrough because it allows to combine several univariate p-boxes into a multivariate p-box [77] that incorporates the epistemic uncertainty coming from copula description. The uncertainty conveyed by empirical margins is investigated in [78] within a framework where the copula PDF is parametric and not subject to imprecise probabilities. On the contrary, the incomplete state of knowledge stemming from an empirical characterization of the dependence structure is studied in [79]. The most advanced contribution has been recently provided in [80] where a hierarchical Bayesian multimodel approach allows to quantify 
uncertainty in model selection and parameter estimation for both the margins and the copula.

\subsection{Reliability-oriented sensitivity analysis}

Identifying which uncertain parameter $\boldsymbol{\Theta}_{i}$ has the strongest impact on the distribution of the failure probability $P_{f}(\boldsymbol{\Theta})$ is one of the main purpose of ROSA. It is different from sensitivity analysis of model output (SAMO) [81] which only focuses on how to fairly share the aleatory uncertainties propagated by the input variables $X_{i}$ on the output $Y$. In that respect, SAMO and ROSA do not provide the same kind of information to analysts. Insights into ROSA techniques are available in $[29,30]$. The way of taking into account the variation range of $\Theta$ gives birth to a split between local and global ROSA methods. Local ROSA methods consider local variations on $P_{f}(\boldsymbol{\Theta})$ due to small deviations from the nominal value $\boldsymbol{\theta}^{\star}=\mathbb{E}[\boldsymbol{\Theta}]$. A well-known approach consists in computing the gradient of the failure probability with respect to input PDF parameters:

$$
\nabla_{\boldsymbol{\theta}} P_{f}\left(\boldsymbol{\theta}^{\star}\right):=\left[\frac{\partial P_{f}}{\partial \theta_{j}}\left(\boldsymbol{\theta}^{\star}\right)\right]_{1 \leq j \leq p} .
$$

The way one should proceed differs according to the reliability algorithm used to estimate $P_{f}\left(\boldsymbol{\theta}^{\star}\right)$. When $\mathrm{CMC}$ is used to estimate $P_{f}\left(\boldsymbol{\theta}^{\star}\right)$, the simulated samples can be post-processed in order to derive all partial derivatives. Indeed, inserting Eq. (10) into Eq. (13) yields:

$$
\nabla_{\boldsymbol{\theta}} P_{f}\left(\boldsymbol{\theta}^{\star}\right)=\mathbb{E}\left[\mathbb{1}_{D_{f}}(\boldsymbol{X}) \nabla_{\boldsymbol{\theta}} \log f_{\boldsymbol{X} \mid \boldsymbol{\Theta}}\left(\boldsymbol{X} \mid \boldsymbol{\theta}^{\star}\right)\right],
$$

and Monte Carlo estimators come naturally $[82,83]$ provided that one is able to access the score function $\nabla_{\boldsymbol{\theta}} \log f_{\boldsymbol{X} \mid \boldsymbol{\Theta}}$. Those local ROSA indices can be extended to the case of dependent inputs where a copula $\mathrm{PDF} c_{\boldsymbol{U}}$ is part of $f_{\boldsymbol{X}}[84,85]$. In the case of adaptive importance sampling [86] and subset simulation [87], similar techniques allow to compute local ROSA indices as byproducts of

$\hat{P}_{f}\left(\boldsymbol{\theta}^{\star}\right)$. Looking for the largest term $\left|\frac{\partial P_{f}}{\partial \theta_{j}}\left(\boldsymbol{\theta}^{\star}\right)\right|$ gives some idea of the input PDF parameter $\theta_{j}$ that must be investigated with the greatest of care before postulating the design value $\boldsymbol{\theta}^{\star}$.

Local ROSA only provides a partial understanding of how $P_{f}(\boldsymbol{\Theta})$ behaves in response to uncertain parameters $\Theta$. Local ROSA indices are only relevant in the vicinity of $\boldsymbol{\theta}^{\star}$ and have to be updated as soon as another design point is considered. An intuitive extension of the local ROSA indices is proposed in [88] where each partial derivative is integrated over its univariate variation range $D_{\theta_{j}}$ :

$$
\mathrm{SP}_{j}\left(\boldsymbol{\theta}^{\star}\right):=\int_{D_{\theta_{j}}}\left|\frac{\partial P_{f}}{\partial \theta_{j}}\left(\theta_{1}^{\star}, \ldots, \theta_{j-1}^{\star}, \theta_{j}, \theta_{j+1}^{\star}, \ldots, \theta_{p}^{\star}\right)\right| \mathrm{d} \theta_{j} .
$$

In this method, ROSA is performed in the spirit of the derivative-based global sensitivity measures (DGSM) developed in [89, 90] but it must be noted that the indices $\mathrm{SP}_{j}\left(\boldsymbol{\theta}^{\star}\right)$ obey a slighlty different definition. Remembering that global ROSA is supposed to emancipate from the design point $\boldsymbol{\theta}^{\star}$, this approach does not completely satisfy the expectations because each index $\mathrm{SP}_{j}$ still depends on 
$\boldsymbol{\theta}_{-j}^{\star}:=\left[\theta_{k}^{\star}\right]_{k \neq j}$. A more convincing methodology to perform global ROSA may be to consider the high-dimensional model representation (HDMR) of $P_{f}(\boldsymbol{\Theta})$ according to what is stated in [91]:

$$
P_{f}(\Theta)=\Psi_{0}+\sum_{i=1}^{p} \Psi_{i}\left(\Theta_{i}\right)+\sum_{i<j} \Psi_{i j}\left(\Theta_{i}, \Theta_{j}\right)+\ldots+\Psi_{1 \ldots p}\left(\Theta_{1}, \ldots, \Theta_{p}\right) .
$$

$P_{f}(\boldsymbol{\Theta})$ is thus split down into a sum of $2^{r}-1$ terms. When one seeks to decompose $P_{f}(\boldsymbol{\Theta})$, there are infinitely many solutions to identify the functions $\Psi_{\boldsymbol{u}}$. To ensure unicity within the HDRM decomposition, an orthogonality constraint has to be enforced and this gives birth to the ANOVA decomposition (also called RS-HDMR decomposition) [92] where:

$$
\Psi_{0}=\mathbb{E}\left[P_{f}(\boldsymbol{\Theta})\right] \quad \text { and } \quad \Psi_{\boldsymbol{u}}\left(\boldsymbol{\Theta}_{\boldsymbol{u}}\right)=\mathbb{E}\left[P_{f}(\boldsymbol{\Theta}) \mid \boldsymbol{\Theta}_{\boldsymbol{u}}\right]+\sum_{\boldsymbol{v} \subset \boldsymbol{u}}(-1)^{|\boldsymbol{u}|-|\boldsymbol{v}|} \mathbb{E}\left[P_{f}(\boldsymbol{\Theta}) \mid \boldsymbol{\Theta}_{\boldsymbol{v}}\right] .
$$

Since the variables $\Psi_{\boldsymbol{u}}\left(\boldsymbol{X}_{\boldsymbol{u}}\right)$ are uncorrelated, passing to variance in Eq. (16) yields:

$$
\mathbb{V}\left(P_{f}(\boldsymbol{\Theta})\right)=\sum_{\boldsymbol{v} \subseteq \boldsymbol{u}} \mathbb{V}\left(\Psi_{\boldsymbol{u}}\left(\boldsymbol{\Theta}_{\boldsymbol{u}}\right)\right)
$$

and the "closed" Sobol index associated to the subvector of parameters $\boldsymbol{\Theta}_{u}$ is defined by:

$$
S_{\boldsymbol{u}}=\frac{\mathbb{V}\left(\mathbb{E}\left[P_{f}(\boldsymbol{\Theta}) \mid \boldsymbol{\Theta}_{\boldsymbol{u}}\right]\right)}{\mathbb{V}\left(P_{f}(\boldsymbol{\Theta})\right)}=\frac{\sum_{\boldsymbol{v} \subseteq \boldsymbol{u}} \mathbb{V}\left(\Psi_{\boldsymbol{v}}\left(\boldsymbol{X}_{\boldsymbol{v}}\right)\right)}{\mathbb{V}\left(P_{f}(\boldsymbol{\Theta})\right)}
$$

An obvious drawback lies in the fact that a triple-loop algorithm has to be implemented in order to compute the variance (outer loop), the conditional expectations (in-between loop) and the failure probabilities (inner loop). The "Pick-and-Freeze" estimation scheme [93, 94] allows to relieve all possible methods from the outer loop. The less expensive strategy to estimate Sobol indices is to adopt the nonintrusive stochastic simulation (NISS) framework [95] which has been recently applied in the specific context of ROSA [96, 97]. Indeed, a single simulation loop in the augmented space allows to derive Sobol indices at all orders.

The survey of both local and global ROSA methods reveals two notable weaknesses. On the one hand, the uncertainty due to copula modeling is rarely quantified and has never been compared to the influence of uncertain marginal distributions. Even at the local scale, there is very little work dedicated to measuring the sensitivity of $P_{f}$ to copula parameters. Considering a copula-based input distribution has become a common practice but differentiation with respect to dependence parameters is not deeply investigated, except in [98, 99]. On the other hand, most existing papers only resort to Bayesian priors in order to incorporate epistemic uncertainties. As many modeling choices rather stem from the analysis of field data, the parametric approach is not always fully satisfying. One could prefer resorting to nonparameteric tools but they do not suit the Bayesian framework.

\subsection{Purpose and intent of the paper}

The working assumptions surrounding this article are slightly different from those found in most contributions on the topic. No prior information is available on the input distribution $f_{\boldsymbol{X}}$. A density 
estimation is learnt from a small-sized dataset $\boldsymbol{X}_{\mathrm{obs}} \in \mathbb{R}^{N \times d}$. Epistemic uncertainty does not derive from some Bayesian knowledge but from the sampling uncertainty induced by data collection. In that respect, reliability analysis has to be modified in order to integrate the provided data, which leads to transform both Eq. (10) and (11) into:

$$
\begin{aligned}
& P_{f}\left(\boldsymbol{X}_{\mathrm{obs}}\right)=\mathcal{R}\left(\hat{f}_{\boldsymbol{X}}\left(\cdot \mid \boldsymbol{X}_{\mathrm{obs}}\right)\right)=\int_{\mathbb{R}^{d}} \mathbb{1}_{D_{f}}(\boldsymbol{x}) \hat{f}_{\boldsymbol{X}}\left(\boldsymbol{x} \mid \boldsymbol{X}_{\mathrm{obs}}\right) \mathbf{d} \boldsymbol{x} \\
& \hat{P}_{f}\left(\boldsymbol{X}_{\mathrm{obs}}\right)=\mathcal{R}_{w}\left(\hat{f}_{\boldsymbol{X}}\left(\cdot \mid \boldsymbol{X}_{\mathrm{obs}}\right)\right)
\end{aligned}
$$

As a consequence, the estimated failure probability must be understood as the final result of a computational process that performs consecutively statistical learning and rare-event simulation. What is called statistical learning comprises any procedure that relies on the estimation of both the copula function and the margins. As the quantity of interest can be expressed in terms of the estimated marginal distributions $\hat{f}_{X_{j}}\left(\cdot \mid \boldsymbol{X}_{\text {obs }}\right)$ and the estimated copula $\hat{c}_{\boldsymbol{U}}\left(\cdot \mid \boldsymbol{X}_{\mathrm{obs}}\right)$, the question that arises here is to identify which estimated function has the strongest influence on data-driven reliability assessment. Therefore, the main motivation of this work is to fairly quantify the share of epistemic uncertainties conveyed by the various components of the input probabilistic model. The authors define appropriate ROSA indices and they develop a data-driven algorithm to estimate them accurately. Having access to this kind of sensitivity indicators allows to identify which entity within the estimated joint distribution deserves a finer statistical treatment. This takes its own full meaning in practice when the analyst is only given one single dataset $\boldsymbol{x}_{\mathrm{obs}}$ and hopes to reduce as much as possible the uncertainty in reliability assessment. If one marginal distribution $\hat{f}_{X_{j_{0}}}$ is targeted as the most sensitive to small changes affecting data, soliciting expert judgement about $f_{X_{j_{0}}}$ or requiring additional data for $X_{j_{0}}$ may appear as a softer solution than collecting other joint observations. Such a practical context goes hand in hand with problems that make global ROSA indices hard to compute. Since the starting material is limited to the given state of knowledge $\left\{\boldsymbol{X}_{\mathrm{obs}}=\boldsymbol{x}_{\mathrm{obs}}\right\}$, only one realization of the margins $\hat{f}_{X_{j}}\left(\cdot \mid \boldsymbol{X}_{\mathrm{obs}}\right)$, the copula $\hat{c}_{\boldsymbol{U}}\left(\cdot \mid \boldsymbol{X}_{\mathrm{obs}}\right)$ and the failure probability $P_{f}\left(\boldsymbol{X}_{\mathrm{obs}}\right)$ can be derived. Moreover, the joint distribution of functional inputs is unknown and cannot be assessed with traditional uncertainty quantification tools such as Karhunen-Loève expansions. The choice of appropriate sensitivity indices and the simulation of functional inputs are left open and are then examined thoroughly in this work. In addition, these problems are compounded by the computational burden entailed by the large number of failure probabilities that must be estimated to perform global ROSA. In Section 3, the main foundations of the proposed methodology are laid in the case of exact reliability assessment $\mathcal{R}$. Then, in Section 4 , the method is adapted to the case where rare-event simulation $\mathcal{R}_{w}$ is used to estimate the failure probability. 


\section{Step-by-step construction of the ROSA procedure}

In this section, everything is done as if $\mathcal{R}$ could be performed exactly and without incurring any computational cost. Of course, those assumptions are unrealistic because exactness in reliability assessment can only occur in very specific situations where $\phi(\cdot)$ and $f_{\boldsymbol{X}}$ are excessively simple. However, the aim of this section is above all to answer the two following thorny questions. Firstly, there is a need to better describe the probabilistic nature of data-driven epistemic uncertainties. It means being able to understand the uncertainty transfer from $\boldsymbol{X}_{\text {obs }}$ to $P_{f}\left(\boldsymbol{X}_{\text {obs }}\right)$ as well as being able to simulate $\boldsymbol{X}_{\text {obs }}$ by means of the given dataset $\boldsymbol{x}_{\mathrm{obs}} \in \mathbb{R}^{N \times d}$. Secondly, a major hurdle is to know how the uncertainty induced on $P_{f}\left(\boldsymbol{X}_{\text {obs }}\right)$ may be divided into one share due to uncertainty on the copula and others due to uncertainty on the margins. The proposed method is then applied to a simple use case where both $f_{\boldsymbol{X}}$ and $\phi(\cdot)$ satisfy the hypotheses of this section. The difficulties inherent to reliability analysis will be studied in Section 4 and solutions to address them will be put forth at this point.

\subsection{About data-driven epistemic uncertainty}

\subsubsection{Sample-based state of knowledge}

The provided dataset $\boldsymbol{x}_{\mathrm{obs}}$ is a $N$-sample of the input vector $\boldsymbol{X} \sim \mu_{\boldsymbol{X}}$ and may be seen as a realization of the following random matrix $\boldsymbol{X}_{\text {obs }}$ :

$$
\boldsymbol{X}_{\mathrm{obs}}=\left[X_{j}^{(i)}\right]_{\substack{1 \leq i \leq N \\
1 \leq j \leq d}}=\left(\begin{array}{cccccc}
X_{1}^{(1)} & X_{2}^{(1)} & \ldots & X_{j}^{(1)} & \ldots & X_{d}^{(1)} \\
X_{1}^{(2)} & X_{2}^{(2)} & \ldots & X_{j}^{(2)} & \ldots & X_{d}^{(2)} \\
\vdots & \vdots & & \vdots & & \vdots \\
X_{1}^{(i)} & X_{2}^{(i)} & \ldots & X_{j}^{(i)} & \ldots & X_{d}^{(i)} \\
\vdots & \vdots & & \vdots & & \vdots \\
X_{1}^{(N)} & X_{2}^{(N)} & \ldots & X_{j}^{(N)} & \ldots & X_{d}^{(N)}
\end{array}\right) \sim\left(\mu_{\boldsymbol{X}}\right)^{\otimes N}
$$

If extracted from $\boldsymbol{X}_{\text {obs }}$, the $i$-th row is denoted by $\boldsymbol{X}_{\mathrm{obs}}^{[i \boldsymbol{\bullet}]}$ and is one observation of $\boldsymbol{X}$ :

$$
\boldsymbol{X}_{\mathrm{obs}}^{[i \bullet]}=\left[X_{j}^{(i)}\right]_{1 \leq j \leq d}=\left(X_{1}^{(i)}, \ldots, X_{d}^{(i)}\right) \sim \mu_{\boldsymbol{X}} .
$$

Furthermore, the $j$-th column is denoted by $\boldsymbol{X}_{\mathrm{obs}}^{[\bullet j]}$ and consists of a $N$-sample of the variable $X_{j}$ which obeys the marginal distribution $\mu_{X_{j}}$ :

$$
\boldsymbol{X}_{\mathrm{obs}}^{[\bullet j]}=\left[X_{j}^{(i)}\right]_{1 \leq i \leq N}=\left(\begin{array}{c}
X_{j}^{(1)} \\
\vdots \\
X_{j}^{(N)}
\end{array}\right) \sim\left(\mu_{X_{j}}\right)^{\otimes N} .
$$

To conform with the "small data" context, $N \leq 500$ is assumed throughout this paper. Being able to estimate the multivariate input PDF from a small amount of data is a daunting challenge that 
may deeply impact the robustness of reliability assessment. As it is encountered in many application fields [100, 101, 102], this issue has paved the way to several methodological research works [31, 32, 33]. By virtue of Sklar's theorem, a multivariate joint PDF can be constructed by capturing separately univariate behaviours and dependence properties. In regard to this, let $\mathcal{M}_{1}, \ldots, \mathcal{M}_{d}$ and $\mathcal{M}_{\text {cop }}$ be the distribution models retained to learn the margins and the copula from data. On top of that, $\mathcal{M}$ stands for the resulting overall distribution model obtained after bringing all $d+1$ components together. Thus, statistical learning $\mathcal{L}$ may be summed up as follows:

$$
\begin{aligned}
\mathcal{L}: \mathbb{R}^{N \times d} & \longrightarrow \mathbb{L}^{2}\left(\mathbb{R}^{d}\right) \\
\boldsymbol{x}_{\mathrm{obs}} & \longmapsto \hat{f}_{\boldsymbol{X}}\left(\cdot \mid \mathcal{M}, \boldsymbol{x}_{\mathrm{obs}}\right)
\end{aligned}
$$

with:

$$
\hat{f}_{\boldsymbol{X}}\left(\boldsymbol{x} \mid \mathcal{M}, \boldsymbol{x}_{\mathrm{obs}}\right)=\left[\prod_{j=1}^{d} \hat{f}_{X_{j}}\left(x_{j} \mid \mathcal{M}_{j}, \boldsymbol{x}_{\mathrm{obs}}\right)\right] \times \hat{c}_{\boldsymbol{U}}\left(u_{1}, \ldots, u_{d} \mid \mathcal{M}_{\mathrm{cop}}, \boldsymbol{x}_{\mathrm{obs}}\right)
$$

and:

$$
u_{j}=\hat{F}_{X_{j}}\left(x_{j} \mid \mathcal{M}_{j}, \boldsymbol{x}_{\mathrm{obs}}\right)=\int_{-\infty}^{x_{j}} \hat{f}_{X_{j}}\left(t \mid \mathcal{M}_{j}, \boldsymbol{x}_{\mathrm{obs}}\right) \mathrm{d} t .
$$

Statistical learning $\mathcal{L}$ is a two-step process including model selection and data calibration. Once $\mathcal{M}$ is chosen, calibration $\mathcal{C}_{\mathcal{M}}$ can be done with maximum likelihood estimation (MLE), the method of moments, the maximum entropy principle or any other adequate inference procedure. Among epistemic uncertainties, the part that can be attributed to the lack of knowledge about the distribution type is called model uncertainty. In the absence of model uncertainty, $f_{\boldsymbol{X}}$ cannot however be entirely reconstructed because of data scarcity. The uncertainty conveyed by the fit is known as data uncertainty. The more operational data are gathered to build $\boldsymbol{x}_{\mathrm{obs}}$, the greater data uncertainty can be shrunk. Analyzing how epistemic uncertainties are impacted by both the choice of the distribution type and the estimation of parameters is studied in [103].

In practice, as $\mathcal{M}_{\mathrm{th}}$ is often unknown, model selection is influenced, if not completely monitored, by a prior data analysis (in other words by $\boldsymbol{x}_{\mathrm{obs}}$ itself). Data-driven model selection $\mathcal{S}$ includes countless strategies ranging from performing an elementary hypothesis testing to maximizing an information criterion [104] in order to find the best-fitted model $\mathcal{M}^{\star}$ within a set of $r$ candidates:

$$
\begin{aligned}
\mathcal{S}: \mathbb{R}^{N \times d} & \longrightarrow\left\{\mathcal{M}^{(1)}, \ldots, \mathcal{M}^{(r)}\right\} \\
\boldsymbol{x}_{\mathrm{obs}} & \longmapsto
\end{aligned}
$$

As a consequence, model uncertainty and data uncertainty are not so easy to separate. As shown in Figure 1, they are intimately linked at the core of the inferential process and, in all what follows, they will be addressed as a single entity (referred to as epistemic uncertainty) carrying all the uncertainty due to the imperfect state of knowledge about the input distribution $f_{\boldsymbol{X}}$. Even if the ROSA procedure 


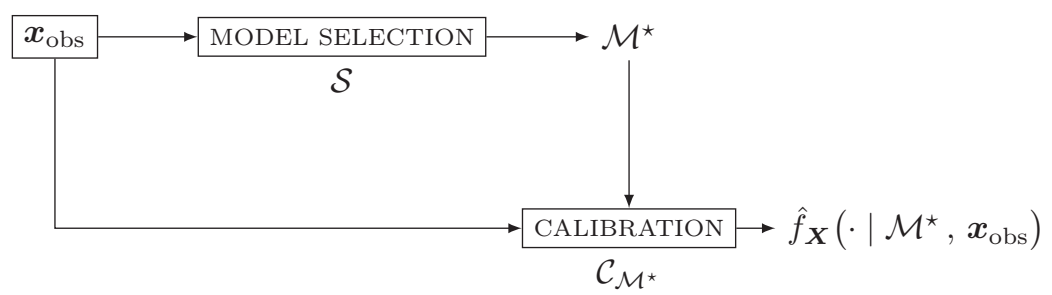

Figure 1: Decomposition of epistemic uncertainty during statistical learning

developed in this paper can be applied to any multivariate model $\mathcal{M}$ with an explicit copula-marginal separation, it is strongly recommended to pay close attention to model choices. As far as the margins are concerned, distribution models that offer some robustness to multimodality and significant accuracy in the tails are particularly suitable. Besides, copula learning is often deemed satisfactory if it manages to reconcile asymmetry, polymorphism and computational tractability.

\subsubsection{From sampling uncertainty to uncertainty in reliability assessment}

Reliability assessment in presence of incomplete probabilistic information follows a data-driven two-phased process which is made possible by a combined use of statistical learning $\mathcal{L}$ and rare-event simulation $\mathcal{R}$. Data-driven reliability assessment is denoted by $\mathcal{D}$ and can be formalized in terms of the given dataset $\boldsymbol{x}_{\mathrm{obs}}$ as follows:

$$
\begin{aligned}
P_{f}\left(\boldsymbol{x}_{\mathrm{obs}}\right) & =\mathbb{P}\left\{\boldsymbol{X} \in D_{f} \mid \boldsymbol{X}_{\mathrm{obs}}=\boldsymbol{x}_{\mathrm{obs}}\right\}, \\
& =\int_{\mathbb{R}^{d}} 1_{D_{f}}(\boldsymbol{x}) \hat{f}_{\boldsymbol{X}}\left(\boldsymbol{x} \mid \boldsymbol{x}_{\mathrm{obs}}\right) \mathbf{d} \boldsymbol{x}=\int_{\mathbb{R}^{d}} 1_{D_{f}}(\boldsymbol{x})\left[\left(\mathcal{L}\left(\boldsymbol{x}_{\mathrm{obs}}\right)\right)(\boldsymbol{x})\right] \mathrm{d} \boldsymbol{x}, \\
& =[\mathcal{R} \circ \mathcal{L}]\left(\boldsymbol{x}_{\mathrm{obs}}\right):=\mathcal{D}\left(\boldsymbol{x}_{\mathrm{obs}}\right) .
\end{aligned}
$$

The question is then to understand how different the learnt PDF $\mathcal{L}\left(\boldsymbol{x}_{\text {obs }}\right)$ and the reliability estimation $P_{f}\left(\boldsymbol{x}_{\mathrm{obs}}\right)$ would have been if another small-sized dataset simulated from $\mu_{\boldsymbol{X}}$ had been supplied. As $\boldsymbol{X}_{\mathrm{obs}}$ is made up of time-invariant independent observations, it is characterized by the $N$-tensored product measure $\left(\mu_{\boldsymbol{X}}\right)^{\otimes N}$. Since $\mathcal{R}$ and $\mathcal{L}$ are deterministic operators, the uncertainty transfer gives birth to pushforward measures [105] for all ensuing random entities. The consecutive steps are described in Figure 2. As each functional component of Sklar's theorem can be learnt separately from all others, an image measure is defined for each one. The measures associated to any $\hat{f}_{X_{j}}$ and to $\hat{c}_{U}$ are respectively denoted $\mu_{\hat{f}_{j}}$ and $\mu_{\hat{c}}$. When the margins and the copula are reassembled into a multivariate distribution in $\mathbb{R}^{d}$ as stated in Eq. (26), the resulting PDF follows a probability measure denoted by $\mu_{\hat{f}}$. Finally, at the endpoint of uncertainty propagation, the measure $\mu_{P_{f}}$ governs how the failure probability $P_{f}\left(\boldsymbol{X}_{\mathrm{obs}}\right)$ behaves in response to $\mu_{\hat{f}}$. 


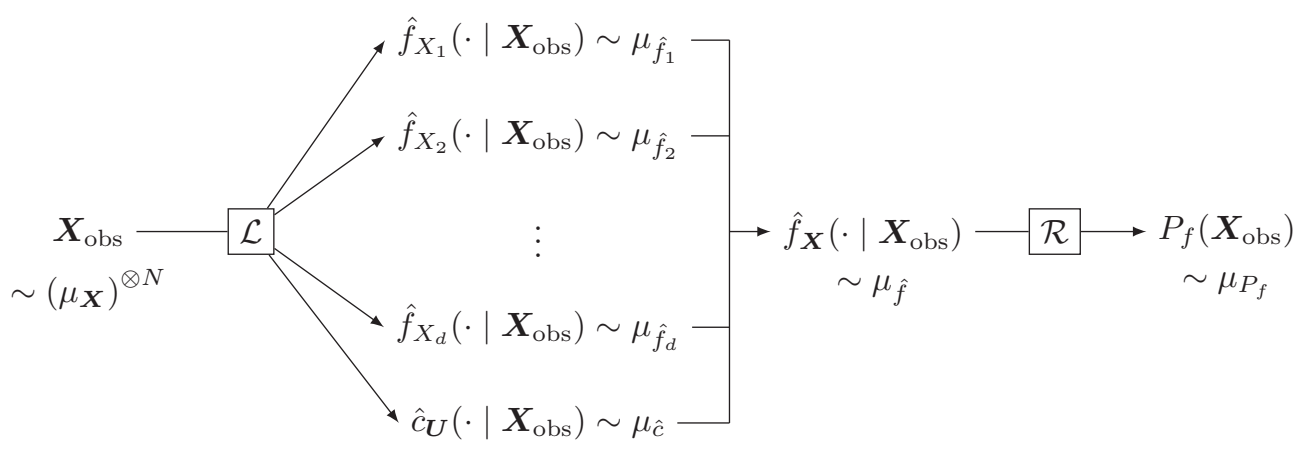

Figure 2: Impact of sampling uncertainty from a probabilistic point of view.

To achieve the objective declared in Section 2.5, one needs to perform SA on $P_{f}\left(\boldsymbol{X}_{\text {obs }}\right)$ with respect to the collection $\mathcal{F}$ which bundles the estimated marginal distributions $\hat{f}_{X_{j}}\left(\cdot \mid \boldsymbol{X}_{\mathrm{obs}}\right)$ and the estimated copula distribution $\hat{c}_{\boldsymbol{U}}\left(\cdot \mid \boldsymbol{X}_{\text {obs }}\right)$. The associated input-output matching $\Psi$ may be set out as follows:

$$
\begin{aligned}
& \Psi: \quad \mathbb{L}^{2}(\mathbb{R}) \times \cdots \times \mathbb{L}^{2}(\mathbb{R}) \times \mathbb{L}^{2}\left(\mathcal{H}_{d}\right) \quad \longrightarrow \quad[0,1] \\
& \mathcal{F}:=\bigcup_{j=1}^{d}\left\{\hat{f}_{X_{j}}\left(\cdot \mid \boldsymbol{X}_{\mathrm{obs}}\right)\right\} \cup\left\{\hat{c}_{\boldsymbol{U}}\left(\cdot \mid \boldsymbol{X}_{\mathrm{obs}}\right)\right\} \longmapsto P_{f}\left(\boldsymbol{X}_{\mathrm{obs}}\right)
\end{aligned}
$$

$\Psi$ estimates the failure probability with a starting material consisting of a disassembled joint distribution. The major difficulty encountered when attempting to conduct ROSA on $\Psi$ is the lack of knowledge about the probabilistic distribution of the input functional objects. The only available thing is one dataset $\boldsymbol{x}_{\mathrm{obs}}$, the associated input PDF $\hat{f}_{\boldsymbol{X}}\left(\cdot \mid \boldsymbol{x}_{\mathrm{obs}}\right)$ and the resulting failure probability $P_{f}\left(\boldsymbol{x}_{\mathrm{obs}}\right)$. Issues arising from such a situation are twofold. On the one hand, a discussion is needed about the choice of relevant sensitivity indicators. On the other hand, for any retained SA technique, it will be necessary to produce new observations of the input functional objects, which seems impossible at this stage. These two questions are respectively addressed in Sections 3.2 and 3.3 while Section 3.4 is devoted to computational aspects.

\subsection{Computation of global sensitivity indices}

\subsubsection{Sobol indices applied to functional inputs}

At first sight, $\Psi$ takes $d+1$ functional inputs that may be dependent. As one ambitions to take into account the whole variation range induced by statistical learning $\mathcal{L}$ on the components of the joint distribution, a global sensitivity analysis (GSA) is necessary. For an exhaustive review of GSA methods, the reader is referred to [106]. Sobol indices [91] are variance-based sensitivity measures and they owe their long time popularity to their easy interpretability as well as their large permissiveness. 
Mathematically speaking, they can be applied for any mapping $\Psi$ of the following type:

$$
\begin{aligned}
\Psi: \quad E:=E_{1} \times \cdots \times E_{r} & \rightarrow \mathbb{R} \\
\left(g_{1}, \ldots, g_{r}\right) & \mapsto p
\end{aligned}
$$

provided that the product space $E$ is measurable and that the output variable is square integrable [107]. Sobol indices can thus be extended to the case where $E$ is a functional space [108]. Under the assumption that all input variables $G_{1}, \ldots, G_{r}$ are mutually independent, the first-order Sobol indices are defined by:

$$
S_{j}:=\frac{\mathbb{V}\left(\mathbb{E}\left[P \mid G_{j}\right]\right)}{\mathbb{V}(P)} .
$$

One can notice that $S_{j}$ does not take into account the effect of possible interactions between $G_{j}$ and the complementary set of functional inputs. To do so, one has to compute total-effect Sobol indices but it is beyond the scope of this paper. Moreover, basic calculations in probability enable to express the variance of the conditional expectation as a covariance:

$$
\mathbb{V}\left(\mathbb{E}\left[P \mid G_{j}\right]\right)=\operatorname{Cov}\left(P, P^{j}\right) \text { with }\left\{\begin{array}{l}
P=\Psi\left(G_{1}, \ldots, G_{d}\right) \\
P^{j}=\Psi\left(G_{1}^{\prime}, \ldots, G_{j-1}^{\prime}, G_{j}, G_{j+1}^{\prime}, \ldots, G_{d}^{\prime}\right)
\end{array},\right.
$$

where $G_{k}^{\prime}$ is an independent copy of $G_{k}$ picked up from $\mu_{\boldsymbol{X}_{k}}$ while $G_{j}$ is frozen [93]. This formula paved the way to the "Pick-and-Freeze" estimation technique which allows to transform the initial problem into a simple linear regression one.

Knowing that those indices are likely to be applied to the mapping $\Psi$ described in Eq. (32), a damper has to be put on their use. First, this sampling-based SA procedure is only possible if one manages to generate realizations of the desired input probabilistic measure. In addition, in the case of dependent inputs, computations can still be conducted but conclusions drawn from the resulting indices must not be trusted. In Section 3.2.2, it is explained why conventional estimation techniques introduce dependence between functional components. To remedy this problem, a new conceptual framework is laid in Section 3.2.3 and allows to envision functional components as independent entities.

\subsubsection{Further insights into copula estimation}

Let us first consider the case where all the variables $X_{1}, \ldots, X_{d}$ are independent. The columns $\boldsymbol{X}_{\mathrm{obs}}^{[\bullet \boldsymbol{j}]}$ which are extracted from the dataset $\boldsymbol{X}_{\mathrm{obs}}$ are then mutually independent. Then, after learning each marginal distribution with the selected model $\mathcal{M}_{j}$, the resulting functional objects are also independent and may be denoted by $\hat{f}_{X_{j}}\left(\cdot \mid \mathcal{M}_{j}, \boldsymbol{X}_{\text {obs }}^{[\bullet j]}\right)$ since their inference asks for nothing more than the $j$-th column. The copula distribution has not to be learnt. Otherwise, one can say that $\hat{c}_{\boldsymbol{U}}\left(\cdot \mid \boldsymbol{X}_{\text {obs }}\right)$ is constantly equal to the independence copula $c_{\perp}(\boldsymbol{u})=1$. 
Now, let us examine how incorporating a copula distribution upsets the independence between functional objects. It is clear that the copula $c_{U}$ creates dependence between the dataset columns $\boldsymbol{X}_{\mathrm{obs}}^{[\bullet j]}$ and therefore between the estimated marginal distributions $\hat{f}_{X_{j}}\left(\cdot \mid \mathcal{M}_{j}, \boldsymbol{X}_{\mathrm{obs}}^{[\bullet j]}\right)$. On the contrary, the intricacies of the statistical link that exists between the estimated copula function and the estimated margins is unclear and has to be gone through. The reader can find comprehensive information on copula-based density estimation in $[109,110]$. A classical case occurs when both the margins and the copula are parametrized and then constitute a fully parametric distribution model in $\mathbb{R}^{d}$ whose PDF is given by:

$$
f_{\boldsymbol{X}}\left(\boldsymbol{x} \mid \boldsymbol{\theta}_{1}, \ldots, \boldsymbol{\theta}_{d}, \boldsymbol{\alpha}\right)=\left[\prod_{j=1}^{d} f_{X_{j}}\left(x_{j} \mid \boldsymbol{\theta}_{j}\right)\right] \times c_{\boldsymbol{U}}\left(F_{X_{1}}\left(x_{1} \mid \boldsymbol{\theta}_{1}\right), \ldots, F_{X_{d}}\left(x_{d} \mid \boldsymbol{\theta}_{d}\right) \mid \boldsymbol{\alpha}\right) .
$$

The augmented parameter vector $\boldsymbol{\beta}=\left[\boldsymbol{\theta}_{1}, \ldots, \boldsymbol{\theta}_{d}, \boldsymbol{\alpha}\right]$ is estimated by maximizing the following $\log$-likelihood function expressed for the provided $N$-sample found in $\boldsymbol{X}_{\text {obs: }}$ :

$$
\begin{aligned}
& \ell\left(\boldsymbol{\theta}_{1}, \ldots, \boldsymbol{\theta}_{d}, \boldsymbol{\alpha} \mid \boldsymbol{X}_{\mathrm{obs}}\right)=\ldots \\
& \sum_{j=1}^{\sum_{i=1}^{\sum_{i=1}^{N} \log f_{X_{j}}\left(X_{j}^{(i)} \mid \boldsymbol{\theta}_{j}\right)}}+\underbrace{\sum_{i=1}^{N} \log c_{\boldsymbol{U}}\left(F_{X_{1}}\left(X_{1}^{(i)} \mid \boldsymbol{\theta}_{1}\right), \ldots, F_{X_{d}}\left(X_{d}^{(i)} \mid \boldsymbol{\theta}_{d}\right) \mid \boldsymbol{\alpha}\right)}_{\ell_{j}\left(\boldsymbol{\theta}_{j}\right)} .
\end{aligned}
$$

In order to find more computationally attractive alternatives, a method called Inference Functions for Margins (IFM) consists in running a three-phased optimization algorithm [111] that (a) solves the ML optimization problem for each marginal distribution $f_{X_{j}}$ with $\boldsymbol{X}_{\mathrm{obs}}^{[\bullet j]}$, (b) derives the copula pseudo-observations from the estimated parametric marginal CDFs:

$$
\hat{\boldsymbol{U}}_{\mathrm{obs}}=\left[\hat{U}_{j}^{(i)}\right]_{\substack{1 \leq i \leq N \\ 1 \leq j \leq d}} \text { with } \quad \hat{U}_{j}^{(i)}=\hat{F}_{X_{j}}\left(X_{j}^{(i)} \mid \hat{\boldsymbol{\theta}}_{j}\right)
$$

and (c) solves the ML optimization problem for the copula $c_{U}$ with $\hat{\boldsymbol{U}}_{\text {obs. }}$. The pseudo-observations stored in $\hat{\boldsymbol{U}}_{\text {obs }}$ are actually observations of the random vector $\hat{\boldsymbol{U}}$ defined by $\hat{U}_{j}=\hat{F}_{X_{j}}\left(X_{j} \mid \hat{\boldsymbol{\theta}}_{j}\right)$. The main problem related to the IFM method arises when the marginal distribution types are postulated. For instance, if one margin is misspecified, there is a risk of overestimating the degree of dependence actually existing among the data [112]. Note that for a given distribution, the copula is invariant under strictly increasing transformations of the margins [48]. In that sense, $\boldsymbol{X}, \boldsymbol{U}$ and $\hat{\boldsymbol{U}}$ have the same copula but $\boldsymbol{U}$ has uniform margins whereas $\hat{\boldsymbol{U}}$ has not. The pseudo-observations are not exactly distributed according to a copula distribution, unless $\hat{F}_{X_{j}}\left(\cdot \mid \hat{\boldsymbol{\theta}}_{j}\right)=F_{X_{j}}(\cdot)$ and this depends on the learning accuracy. To avoid such a pitfall, $\hat{\boldsymbol{U}}_{\text {obs }}$ may be computed with a nonparametric approach:

$$
\hat{U}_{j}^{(i)}=\hat{F}_{X_{j}}\left(X_{j}^{(i)} \mid \boldsymbol{X}_{\mathrm{obs}}^{[\bullet j]}\right)=\frac{1}{N+1} \sum_{k=1}^{N} \mathbb{1}_{\left\{X_{j}^{(k)} \leq X_{j}^{(i)}\right\}},
$$


where $\hat{F}_{X_{j}}\left(\cdot \mid \boldsymbol{X}_{\text {obs }}^{[\bullet j]}\right)$ is the empirical CDF built from the $j$-th column of $\boldsymbol{X}_{\text {obs }}$. If both the margins and the copula are estimated with parametric distribution models and if $\hat{\boldsymbol{U}}_{\text {obs }}$ is derived from Eq. (39), the inference algorithm is said to be semi-parametric (SP). This method was developed in [113, 114] and then largely popularized. The values taken by any empirically rescaled variable $\hat{U}_{j}$ are the $\frac{i}{N+1}$ for all $i \in\{1, \ldots, N\}$. An extensive simulation study led in [115] shows that the SP method generally performs better than both MLE and IFM. In this paper, all joint densities will be estimated according to the SP procedure because it appears to be a safer practice in presence of model uncertainty.

\subsubsection{Towards an independent characterization of the margins and the copula}

If one were ever seeking to generate new realizations of the studied functional objects, having access to a random generator $\mathcal{G}_{\boldsymbol{X}}$ of the input probabilistic measure $\mu_{\boldsymbol{X}}$ would be a necessary condition, though unrealistic. The generation process that must be repeated is described in Algorithm 1.

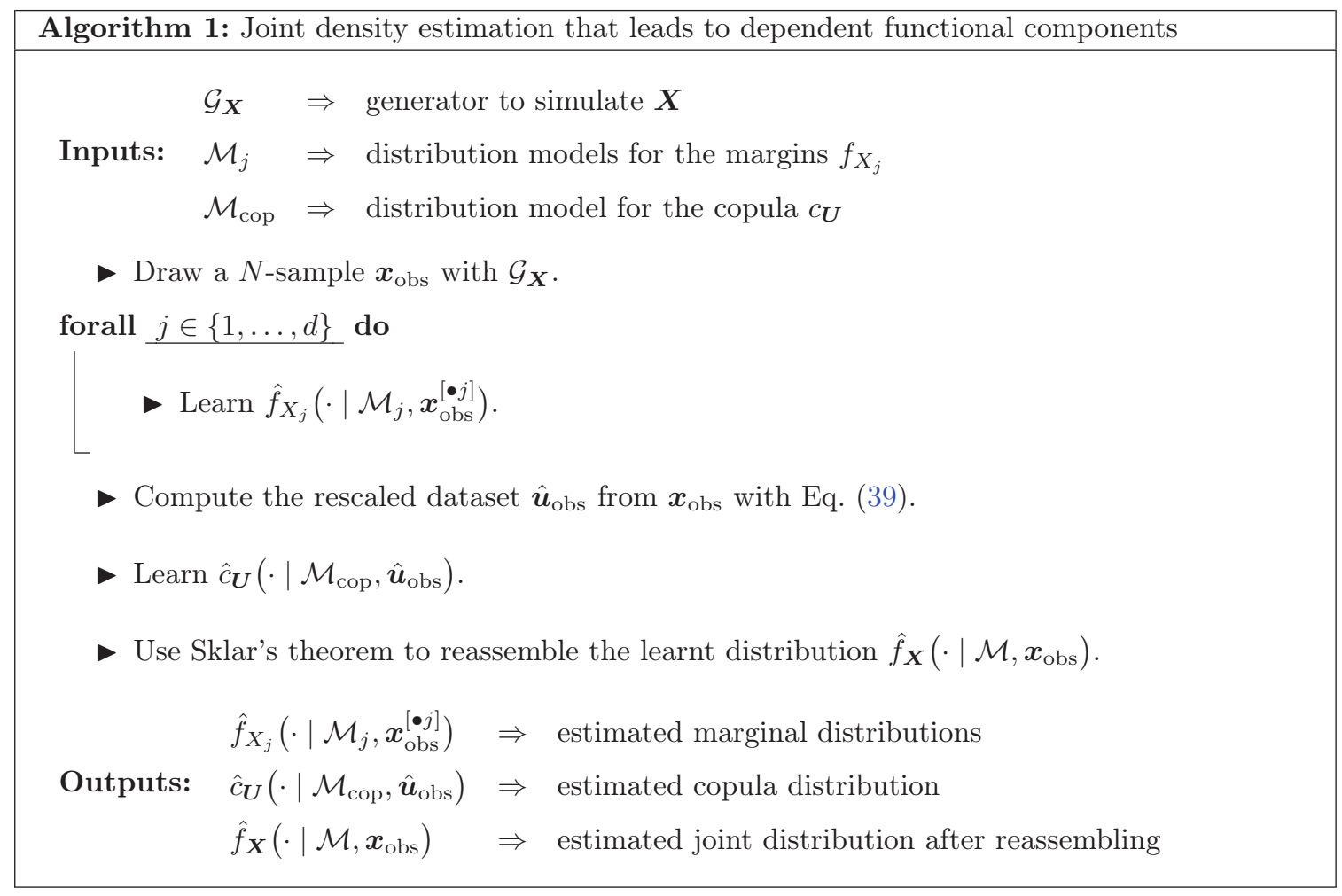

If using the SP technique to perform statistical inference, copula estimation is better isolated because it no longer depends on the estimated margins. However, functional dependence between the copula and the margins still exists since $\boldsymbol{X}_{\mathrm{obs}}^{[\bullet 1]}, \ldots, \boldsymbol{X}_{\mathrm{obs}}^{[\bullet d]}$ and $\hat{\boldsymbol{U}}_{\mathrm{obs}}$ stem from the same dataset $\boldsymbol{X}_{\mathrm{obs}}$. From the SA point of view, the problem would become much simpler if functional components 
evinced mutual independence. This situation is indeed not completely unrealistic. Let us imagine that $\boldsymbol{X}_{\mathrm{obs}}$ is divided into $d+1$ smaller datasets. In order to ensure this, one has to choose $d$ integers $1<n_{1}<\ldots<n_{d}<N$ and has to allocate a certain amount of observations to each learning task:

$$
\begin{gathered}
\boldsymbol{X}_{\mathcal{M}_{1}}=\left\{\boldsymbol{X}_{\mathrm{obs}}^{[i \bullet]}: 1 \leq i \leq n_{1}\right\}, \ldots, \quad \boldsymbol{X}_{\mathcal{M}_{d}}=\left\{\boldsymbol{X}_{\mathrm{obs}}^{[i \bullet}: n_{d-1}+1 \leq i \leq n_{d}\right\}, \\
\boldsymbol{X}_{\mathcal{M}_{\mathrm{cop}}}=\left\{\boldsymbol{X}_{\mathrm{obs}}^{[i \bullet]}: n_{d}+1 \leq i \leq N\right\} .
\end{gathered}
$$

Dividing $\boldsymbol{X}_{\mathrm{obs}}$ into several batches is can not be considered in the "small data" context because each component is then estimated from too little data. Another situation yielding mutual independence among functional components consists in producing independent copies of $\boldsymbol{X}_{\text {obs }}^{[\bullet 1]}, \ldots, \boldsymbol{X}_{\text {obs }}^{[\bullet d]}$ and $\hat{\boldsymbol{U}}_{\text {obs }}$. For this, let us consider the following random variables:

$$
\begin{aligned}
& \boldsymbol{Z}=\left[Z_{1}, \ldots, Z_{d}\right] \in \mathbb{R}^{d} \quad \text { with } \quad f_{\boldsymbol{Z}}(\boldsymbol{z})=\prod_{j=1}^{d} f_{X_{j}}\left(z_{j}\right), \\
& \boldsymbol{V}=\left[V_{1}, \ldots, V_{d}\right] \in \mathcal{H}_{d} \quad \text { with } \quad f_{\boldsymbol{V}}(\boldsymbol{v})=c_{\boldsymbol{U}}(\boldsymbol{v}) \text { and } \boldsymbol{V} \perp \boldsymbol{Z} .
\end{aligned}
$$

$\mathcal{G}_{\boldsymbol{Z}}$ and $\mathcal{G}_{\boldsymbol{V}}$ are the random generators respectively associated to $\boldsymbol{Z}$ and $\boldsymbol{V}$. If statistical learning $\mathcal{L}$ is based on datasets simulated with $\mathcal{G}_{\boldsymbol{Z}}$ and $\mathcal{G}_{\boldsymbol{V}}$, the estimated functional components are independent. Indeed, $\mathcal{G}_{\boldsymbol{Z}}$ produces a random matrix $\boldsymbol{Z}_{\text {obs }} \sim\left(\mu_{\boldsymbol{Z}}\right)^{\otimes N}$ with independent columns $\boldsymbol{Z}_{\text {obs }}^{[\bullet j}$ that exactly work as mutually independent marginal datasets. Moreover, since $\boldsymbol{V}$ is assumed independent of $\boldsymbol{Z}$, $\boldsymbol{V}_{\text {obs }} \sim\left(\mu_{\boldsymbol{V}}\right)^{\otimes N}$ is a dataset consisting of copula samples $\boldsymbol{V}_{\text {obs }}^{[i \bullet}$ which are independent from $\boldsymbol{Z}_{\text {obs }}$. The generation process described in Algorithm 2 leads to an independent collection of functional inputs:

$$
\mathcal{F}_{2}=\left\{\hat{f}_{X_{1}}\left(\cdot \mid Z_{\mathrm{obs}}^{[\bullet 1]}\right), \ldots, \hat{f}_{X_{d}}\left(\cdot \mid \boldsymbol{Z}_{\mathrm{obs}}^{[\bullet d]}\right), \hat{c}_{\boldsymbol{U}}\left(\cdot \mid \boldsymbol{V}_{\mathrm{obs}}\right)\right\}
$$

Hence, the joint behavior of $\mathcal{F}_{2}$ is different from the joint behavior of the initial collection of random objects produced by Algorithm 1:

$$
\mathcal{F}_{1}=\left\{\hat{f}_{X_{1}}\left(\cdot \mid \boldsymbol{X}_{\mathrm{obs}}^{[\bullet 1]}\right), \ldots, \hat{f}_{X_{d}}\left(\cdot \mid \boldsymbol{X}_{\mathrm{obs}}^{[\bullet d]}\right), \hat{c}_{\boldsymbol{U}}\left(\cdot \mid \hat{\boldsymbol{U}}_{\mathrm{obs}}\right)\right\}
$$

The initial uncertainty transfer represented in Figure 2 is modified because of $\mathcal{F}_{2}$ replacing $\mathcal{F}_{1}$ and the new version is shown on Figure 3. The probability measure associated to the reassembled joint density $\hat{f}_{\boldsymbol{X}}\left(\cdot \mid \boldsymbol{Z}_{\text {obs }}, \boldsymbol{V}_{\text {obs }}\right)$ is now denoted by $\mu_{\hat{g}}$. The probability measure assigned to the failure probability $P_{f}\left(\boldsymbol{Z}_{\text {obs }}, \boldsymbol{V}_{\text {obs }}\right)$ is also impacted and becomes $\mu_{Q_{f}}$. Having established this, the ROSA problem seems easier to solve since the adopted point of view allows to envision functional components as mutually independent inputs. Sobol indices may be expressed as follows:

$$
\begin{aligned}
\forall 1 \leq j \leq d, \quad S_{j}:= & \frac{\mathbb{V}\left(\mathbb{E}\left[P_{f}\left(\boldsymbol{Z}_{\mathrm{obs}}, \boldsymbol{V}_{\mathrm{obs}}\right) \mid \hat{f}_{X_{j}}\left(\cdot \mid \boldsymbol{Z}_{\mathrm{obs}}^{[\bullet j]}\right)\right]\right)}{\mathbb{V}\left(P_{f}\left(\boldsymbol{Z}_{\mathrm{obs}}, \boldsymbol{V}_{\mathrm{obs}}\right)\right)}, \\
S_{\mathrm{cop}} & :=\frac{\mathbb{V}\left(\mathbb{E}\left[P_{f}\left(\boldsymbol{Z}_{\mathrm{obs}}, \boldsymbol{V}_{\mathrm{obs}}\right) \mid \hat{c}_{\boldsymbol{U}}\left(\cdot \mid \boldsymbol{V}_{\mathrm{obs}}\right)\right]\right)}{\mathbb{V}\left(P_{f}\left(\boldsymbol{Z}_{\mathrm{obs}}, \boldsymbol{V}_{\mathrm{obs}}\right)\right)},
\end{aligned}
$$




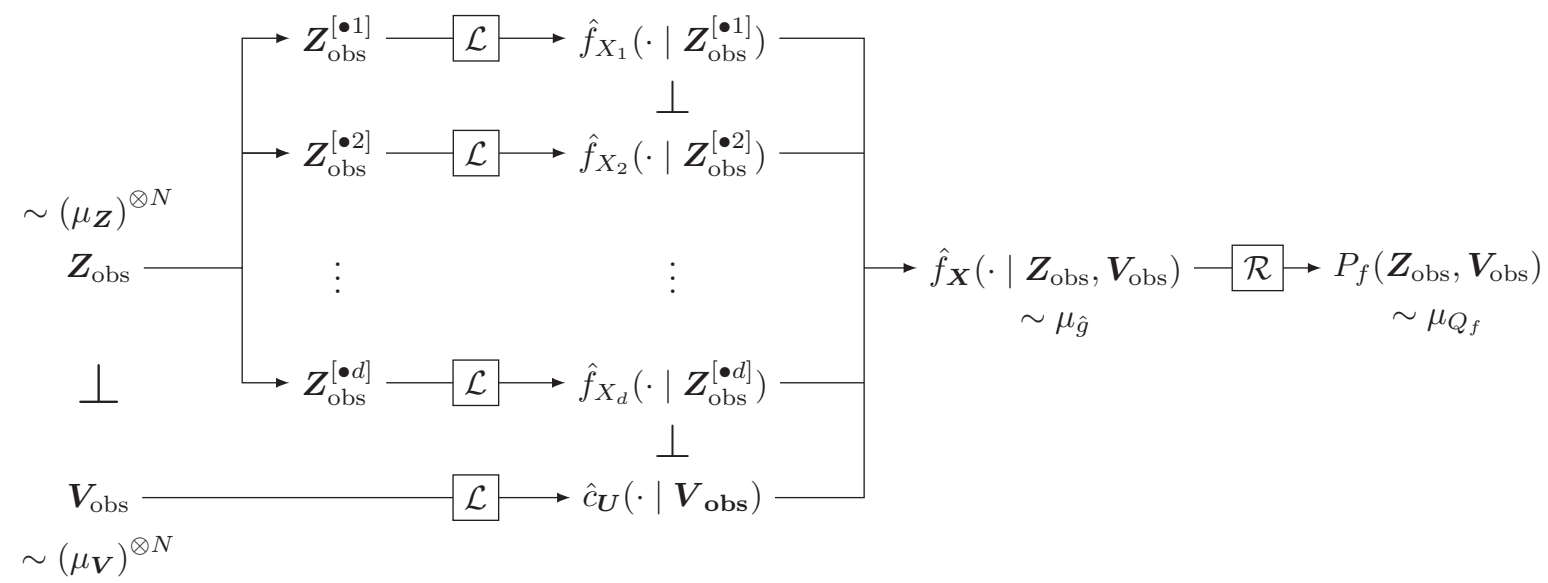

Figure 3: Sampling strategy to achieve functional independence.

with $\left(\boldsymbol{Z}_{\text {obs }}, \boldsymbol{V}_{\text {obs }}\right) \sim\left(\mu_{\boldsymbol{Z}}\right)^{\otimes N} \otimes\left(\mu_{\boldsymbol{V}}\right)^{\otimes N}$. If the choice of Sobol indices as relevant GSA indicators is now justified, the generators $\mathcal{G}_{\boldsymbol{X}}, \mathcal{G}_{\boldsymbol{Z}}$ and $\mathcal{G}_{\boldsymbol{V}}$ remain unavailable. As a result, there is a need to understand how each of them produces samples.

\subsection{How to reproduce unobservable sampling uncertainty?}

When one is only given a small number of observations and then needs to resample according the unknown underlying distribution, a method called the "bootstrap" was developed by Efron [116, 117] and has now become pervasive in statistical applications. Despite its finite size, the set of all observed values $\left\{\boldsymbol{x}_{\mathrm{obs}}^{[i \bullet]}: 1 \leq i \leq N\right\}$ is regarded as the sample space where $\boldsymbol{X}$ lies. Each observation $\boldsymbol{x}_{\mathrm{obs}}^{[i \bullet]}$ becomes an atom of the following discrete probability distribution:

$$
\hat{\mu}\left[\boldsymbol{x}_{\mathrm{obs}}\right]=\frac{1}{N} \sum_{i=1}^{N} \delta_{\boldsymbol{x}_{\mathrm{obs}}^{[i \bullet]}},
$$

where $\delta_{\boldsymbol{x}}$ is the Dirac measure assigned to any $\boldsymbol{x} \in \mathbb{R}^{d}$. Indeed, bootstrapping consists in resampling according to the empirical distribution $\hat{\mu}\left[\boldsymbol{x}_{\mathrm{obs}}\right]$ instead of the true unknown distribution $\mu_{\boldsymbol{X}}$. Simulation with $\hat{\mu}\left[\boldsymbol{x}_{\mathrm{obs}}\right]$ is just uniform sampling (with replacements) from the already observed data. The same initial observation $\boldsymbol{x}_{\mathrm{obs}}^{[k \bullet]}$ may be selected several times and then inserted as many times in the replicated dataset $\tilde{\boldsymbol{x}}_{\text {obs }}$. For comprehensive information about the bootstrap, the reader is advised to consult one of the many reference books on this topic [118, 119]. In SA, bootstrap is often used to derive confidence intervals for Sobol indices by simply swapping the rows of the initial Monte-Carlo design, and therefore without any additional call to $\phi(\cdot)$ [120]. Bootstrap-based SA is often used (for want of anything better) to assess the sensitivity of an output with respect to some input parameters (that 


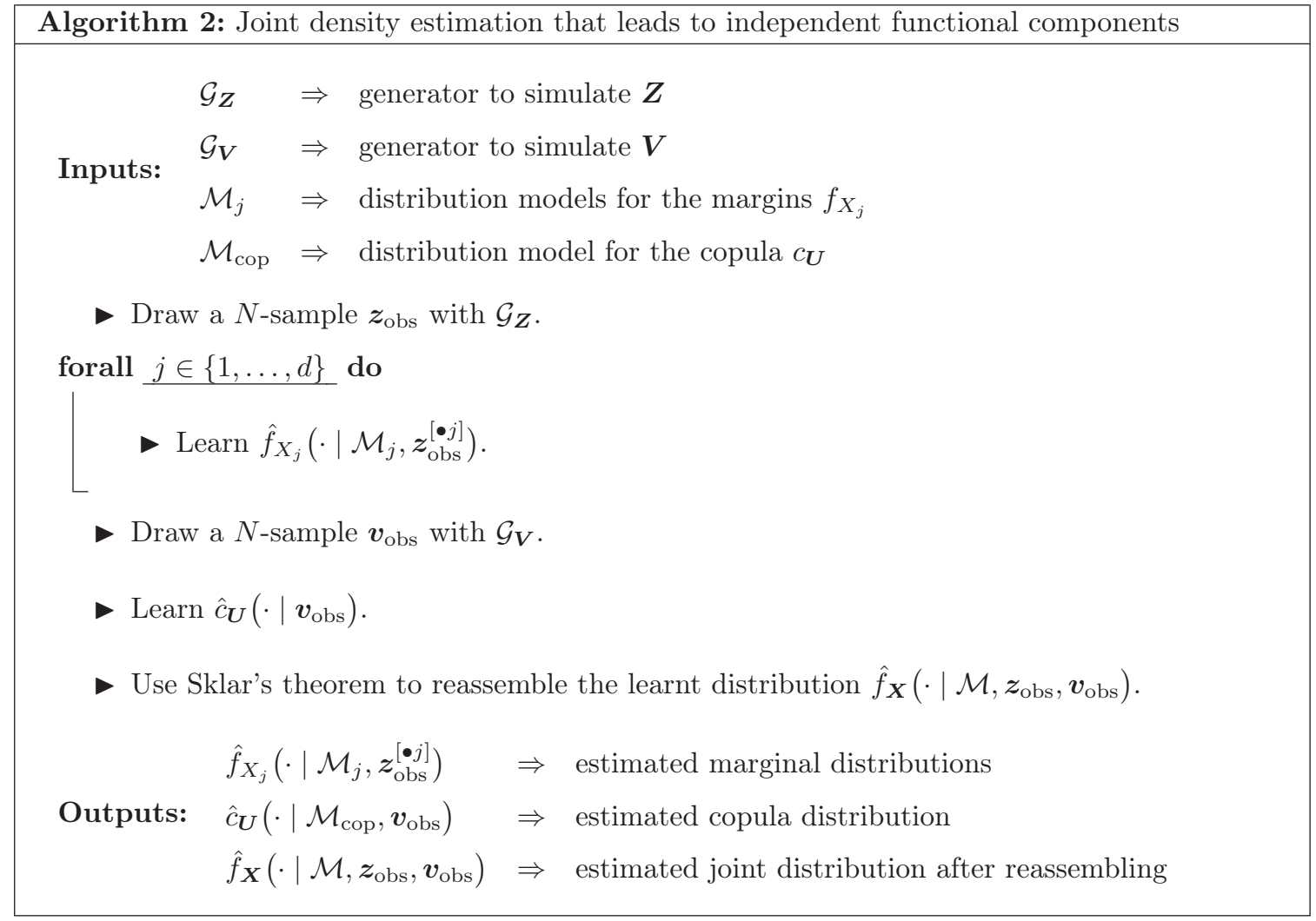


can only be computed once from the initial data). For instance, it is shown in $[121,122]$ that the joint distribution of regression parameters can be assessed from bootstrap replications of the initial dataset. In this work, the context is very similar and the bootstrap is the key element to simulate how the functional inputs are distributed.

Thus, using $\hat{\mu}\left[\boldsymbol{x}_{\mathrm{obs}}\right]$ allows to imitate $\mathcal{G}_{\boldsymbol{X}}$ in Algorithm 1 but it has already been said that it is not exactly what is wanted here since Sobol indices need to be computed from independent functional inputs. To achieve this goal, bootstrap resampling has to be carried out with a slightly different approach, in order to stick as much as possible to what is presented in Algorithm 2. Actually, it is all about understanding how to construct empirical approximations for $\mathcal{G}_{\boldsymbol{Z}}$ and $\mathcal{G}_{\boldsymbol{V}}$. For the given dataset $\boldsymbol{x}_{\mathrm{obs}}$, let us imagine that the matrix coefficients are separated in $d$ batches through a column-by-column split. A univariate empirical distribution $\hat{\mu}\left[\boldsymbol{x}_{\mathrm{obs}}^{[\bullet j]}\right]$ is then attached to each constituted batch. By doing so, the information related to the intrinsic dependence structure are lost and the marginal distributions can then be resampled independently from one another. Mathematically speaking, $\mathcal{G}_{\boldsymbol{Z}}$ is replaced by the $d$-tensored product measure of all marginal empirical distributions:

$$
\hat{\mu}_{\boldsymbol{Z}}\left[\boldsymbol{x}_{\mathrm{obs}}\right]:=\hat{\mu}\left[\boldsymbol{x}_{\mathrm{obs}}^{[\bullet 1]}\right] \otimes \hat{\mu}\left[\boldsymbol{x}_{\mathrm{obs}}^{[\bullet 2]}\right] \otimes \ldots \otimes \hat{\mu}\left[\boldsymbol{x}_{\mathrm{obs}}^{[\bullet d]}\right]
$$

In practice it is nothing but a column-by-column bootstrap resampling procedure based on $\boldsymbol{x}_{\mathrm{obs}}$. The objective is then to reproduce the way the unknown generator $\mathcal{G}_{\boldsymbol{V}}$ works. Copula pseudo-observations $\hat{\boldsymbol{u}}_{\mathrm{obs}}$ are computed from $\boldsymbol{x}_{\mathrm{obs}}$ with Eq. (39) and the empirical measure $\hat{\mu}_{\boldsymbol{V}}\left[\boldsymbol{x}_{\mathrm{obs}}\right]:=\hat{\mu}\left[\hat{\boldsymbol{u}}_{\mathrm{obs}}\right]$ is used to simulate new copula samples. Thus, any bootstrap replication $\tilde{\boldsymbol{v}}$ drawn from $\hat{\boldsymbol{u}}_{\text {obs }}$ may be seen as another training set for copula estimation. The overall resampling mechanism is denoted by $\mathcal{B}$ and allows to simulate a collection $\left\{\tilde{\boldsymbol{z}}_{1}, \ldots, \tilde{\boldsymbol{z}}_{d}, \tilde{\boldsymbol{v}}\right\}$ with the help of the following distribution:

$$
\hat{\mu}_{\boldsymbol{Z} \boldsymbol{V}}\left[\boldsymbol{x}_{\mathrm{obs}}\right]:=\hat{\mu}\left[\boldsymbol{x}_{\mathrm{obs}}^{[\bullet 1]}\right] \otimes \hat{\mu}\left[\boldsymbol{x}_{\mathrm{obs}}^{[\bullet 2]}\right] \otimes \ldots \otimes \hat{\mu}\left[\boldsymbol{x}_{\mathrm{obs}}^{[\bullet d]}\right] \otimes \hat{\mu}\left[\hat{\boldsymbol{u}}_{\mathrm{obs}}\right] .
$$

All matrix manipulations are summarized in Figure 4 where it can be seen that the resampling strategy rests on $d+1$ bootstrap replication steps. In what follows, $\tilde{\boldsymbol{z}}$ denotes the horizontal concatenation of all vectors $\tilde{\boldsymbol{z}}_{j} \in \mathbb{R}^{N}$. Running $\mathcal{B}$ from $\boldsymbol{x}_{\text {obs }}$ provides the ability to simulate independent training sets for the margins and the copula and ultimately provides the ability to simulate independent functional components. Indeed, statistical learning $\mathcal{L}$ transforms any mutually independent collection of replicated samples $\left\{\tilde{\boldsymbol{z}}_{1}, \ldots, \tilde{\boldsymbol{z}}_{d}, \tilde{\boldsymbol{v}}\right\}$ into a mutually independent collection of estimated PDFs $\left\{\hat{f}_{X_{1}}, \ldots, \hat{f}_{X_{d}}, \hat{c}_{\boldsymbol{U}}\right\}$. Algorithm 3 recaps how the bootstrap allows to overcome the major issue of only being given one single dataset $\boldsymbol{x}_{\mathrm{obs}}$. In view of performing GSA on $\Psi$, this resampling algorithm allows to simulate as many input objects as desired. However, since $\mathcal{G}_{\boldsymbol{Z}}$ and $\mathcal{G}_{\boldsymbol{V}}$ are replaced by $\mathcal{B}$, the Sobol indices stated 
in (43) become:

$$
\begin{aligned}
\forall 1 \leq j \leq d, \quad S_{j}^{\mathcal{B}}: & =\frac{\mathbb{V}\left(\mathbb{E}\left[P_{f}(\tilde{\boldsymbol{Z}}, \tilde{\boldsymbol{V}}) \mid \hat{f}_{X_{j}}\left(\cdot \mid \tilde{\boldsymbol{Z}}_{j}\right)\right]\right)}{\mathbb{V}\left(P_{f}(\tilde{\boldsymbol{Z}}, \tilde{\boldsymbol{V}})\right)} \\
S_{\mathrm{cop}}^{\mathcal{B}} & :=\frac{\mathbb{V}\left(\mathbb{E}\left[P_{f}(\tilde{\boldsymbol{Z}}, \tilde{\boldsymbol{V}}) \mid \hat{c}_{\boldsymbol{U}}(\cdot \mid \tilde{\boldsymbol{V}})\right]\right)}{\mathbb{V}\left(P_{f}(\tilde{\boldsymbol{Z}}, \tilde{\boldsymbol{V}})\right)}
\end{aligned}
$$

with $(\tilde{\boldsymbol{Z}}, \tilde{\boldsymbol{V}}) \sim\left(\hat{\mu}_{\boldsymbol{Z} \boldsymbol{V}}\left[\boldsymbol{x}_{\text {obs }}\right]\right)^{\otimes N}$. Writing $\mathcal{B}$ as a superscript of Sobol indices means they are computed with respect to the empirical probability measure $\left(\hat{\mu}_{\boldsymbol{Z} \boldsymbol{V}}\left[\boldsymbol{x}_{\mathrm{obs}}\right]\right)^{\otimes N}$. This notation is omitted when there is no ambiguity on how Sobol indices are computed. The next section explains how to couple all computational bricks in order to estimate the first-order Sobol indices.

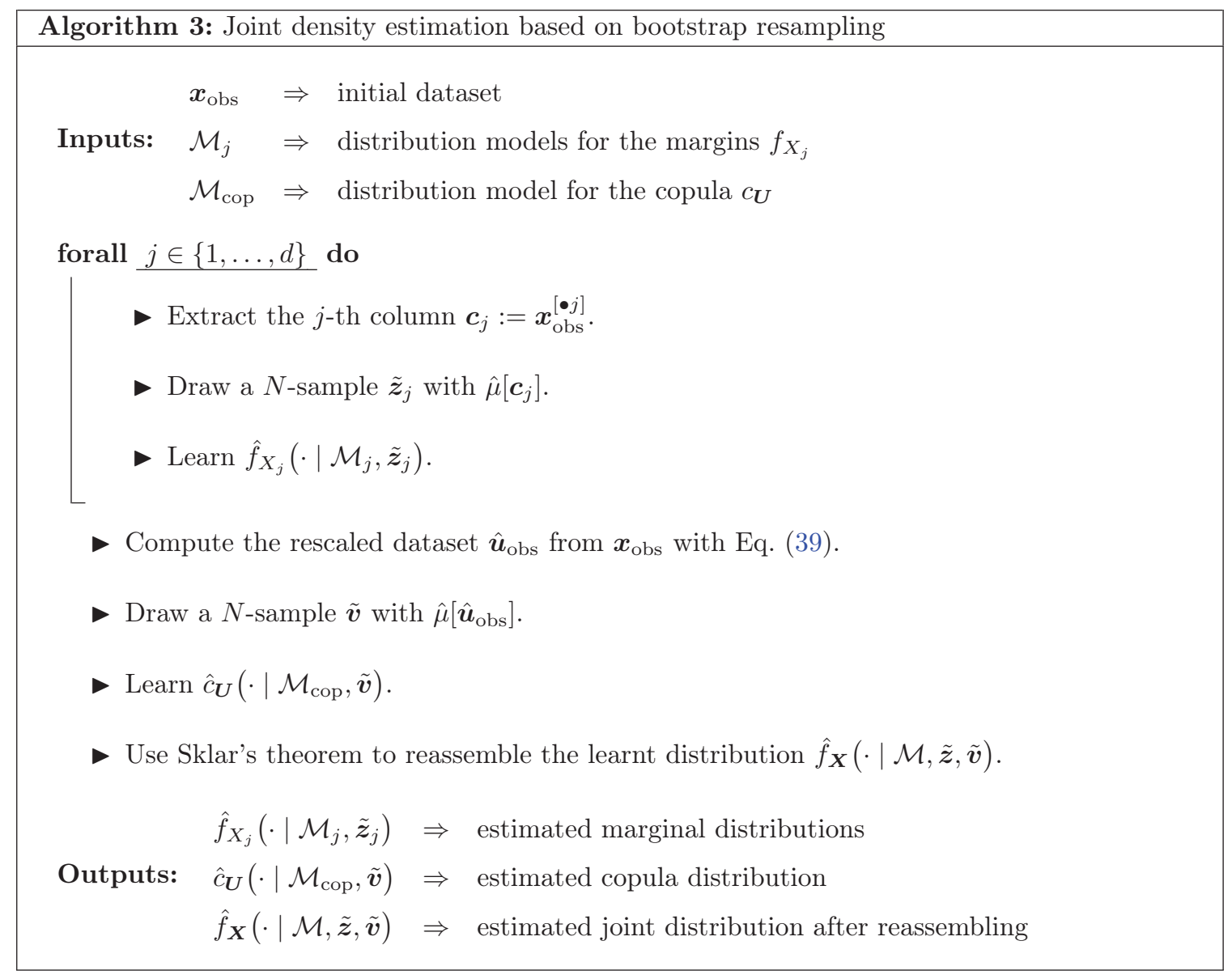

\subsection{Complete workflow}

To perform GSA on $\Psi$, Sobol indices are going to be computed with the "Pick-and-Freeze" estimation scheme denoted by $\mathcal{A}$ and based on Eq. (35). It was proved that the resulting estimators have all desired 


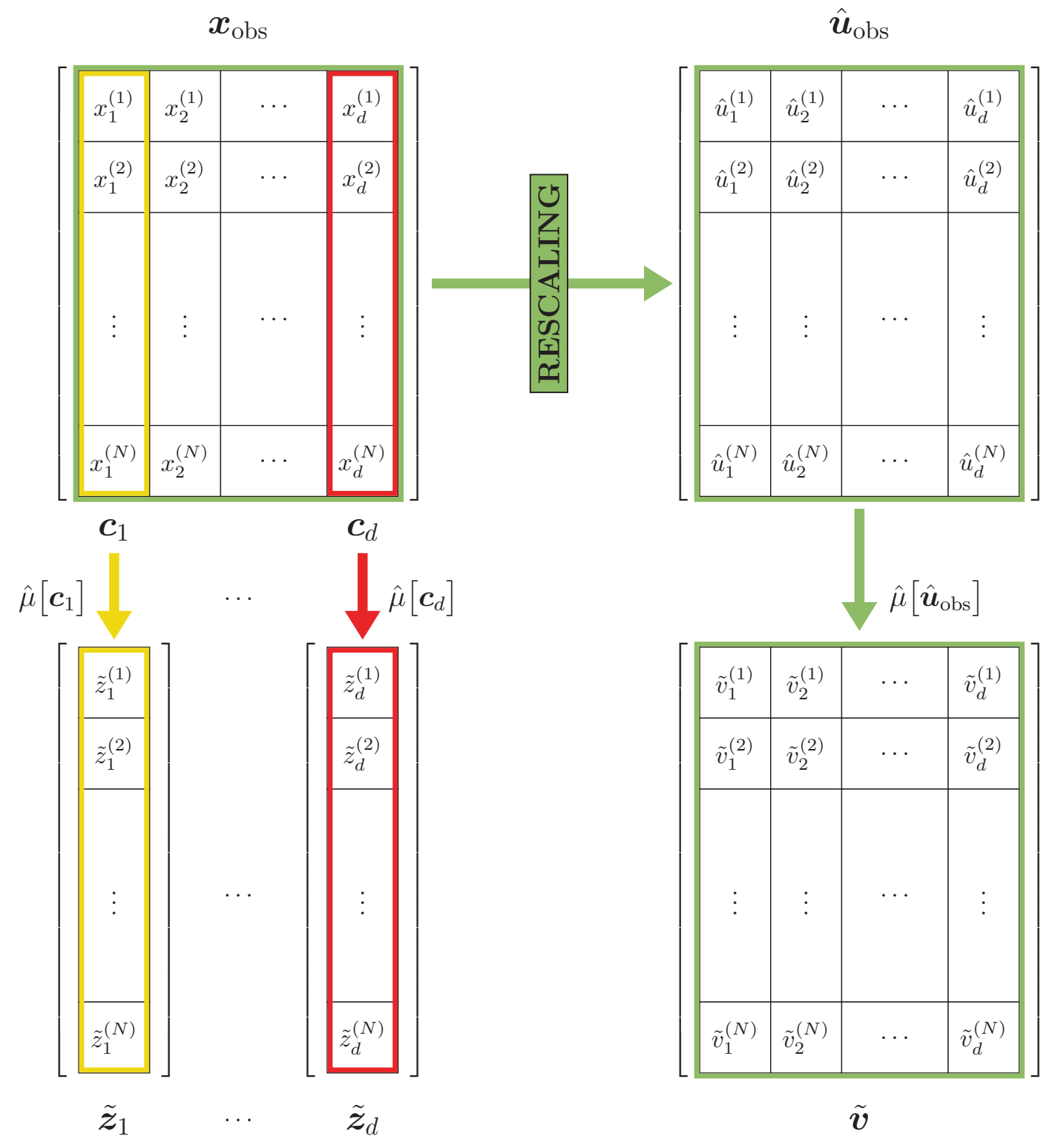

Figure 4: Resampling mechanism $\mathcal{B}$ used to simulate how $\mathcal{G}_{\boldsymbol{Z}}$ and $\mathcal{G}_{\boldsymbol{V}}$ behave. 
statistical properties [107, 123]. From the provided dataset $\boldsymbol{x}_{\mathrm{obs}}$, simulating one input-output sample of the studied mapping $\Psi$ may be achieved as follows:

$$
\begin{aligned}
\boldsymbol{x}_{\mathrm{obs}} \stackrel{\mathcal{B}}{\longmapsto}\{\tilde{\boldsymbol{z}}, \tilde{\boldsymbol{v}}\} \stackrel{\mathcal{L}}{\longmapsto} & \left\{\hat{f}_{X_{1}}, \ldots, \hat{f}_{X_{d}}, \hat{c}_{\boldsymbol{U}}\right\} \stackrel{\text { Sklar }}{\longmapsto} \hat{f}_{\boldsymbol{X}}(\cdot \mid \tilde{\boldsymbol{z}}, \tilde{\boldsymbol{v}}) \stackrel{\mathcal{R}}{\longmapsto} \hat{P}_{f}(\tilde{\boldsymbol{z}}, \tilde{\boldsymbol{v}}) \\
& \left\{\hat{f}_{X_{1}}, \ldots, \hat{f}_{X_{d}}, \hat{c}_{\boldsymbol{U}}\right\} \stackrel{\Psi}{\longmapsto} \hat{P}_{f}(\tilde{\boldsymbol{z}}, \tilde{\boldsymbol{v}})
\end{aligned}
$$

that is by running a three-phased algorithm including:
1. Bootstrap resampling $\mathcal{B}$ :
$\mathbb{R}^{N \times d} \longrightarrow \mathbb{R}^{N} \times \ldots \times \mathbb{R}^{N} \times[0,1]^{N \times d}$
2. Statistical learning $\mathcal{L}$
$\mathcal{L}: \quad \mathbb{R}^{N} \times \ldots \times \mathbb{R}^{N} \times[0,1]^{N \times d} \longrightarrow \mathbb{L}^{2}(\mathbb{R}) \times \ldots \times \mathbb{L}^{2}(\mathbb{R}) \times \mathbb{L}^{2}\left(\mathcal{H}_{d}\right)$,
3. Reliability assessment $\mathcal{R}: \mathbb{L}^{2}(\mathbb{R}) \times \ldots \times \mathbb{L}^{2}(\mathbb{R}) \times \mathbb{L}^{2}\left(\mathcal{H}_{d}\right) \longrightarrow[0,1]$.

The complete workflow with all three computational bricks is shown in Figure 5. Data collection $\mathcal{C}_{\text {obs }}$ is a preliminary step that supplies $\boldsymbol{x}_{\text {obs }}$. It is carried out once and for all. On the contrary, the sequential execution of $\mathcal{B}, \mathcal{L}$ and $\mathcal{R}$ must be done as many times as the chosen sampling-based GSA method demands.

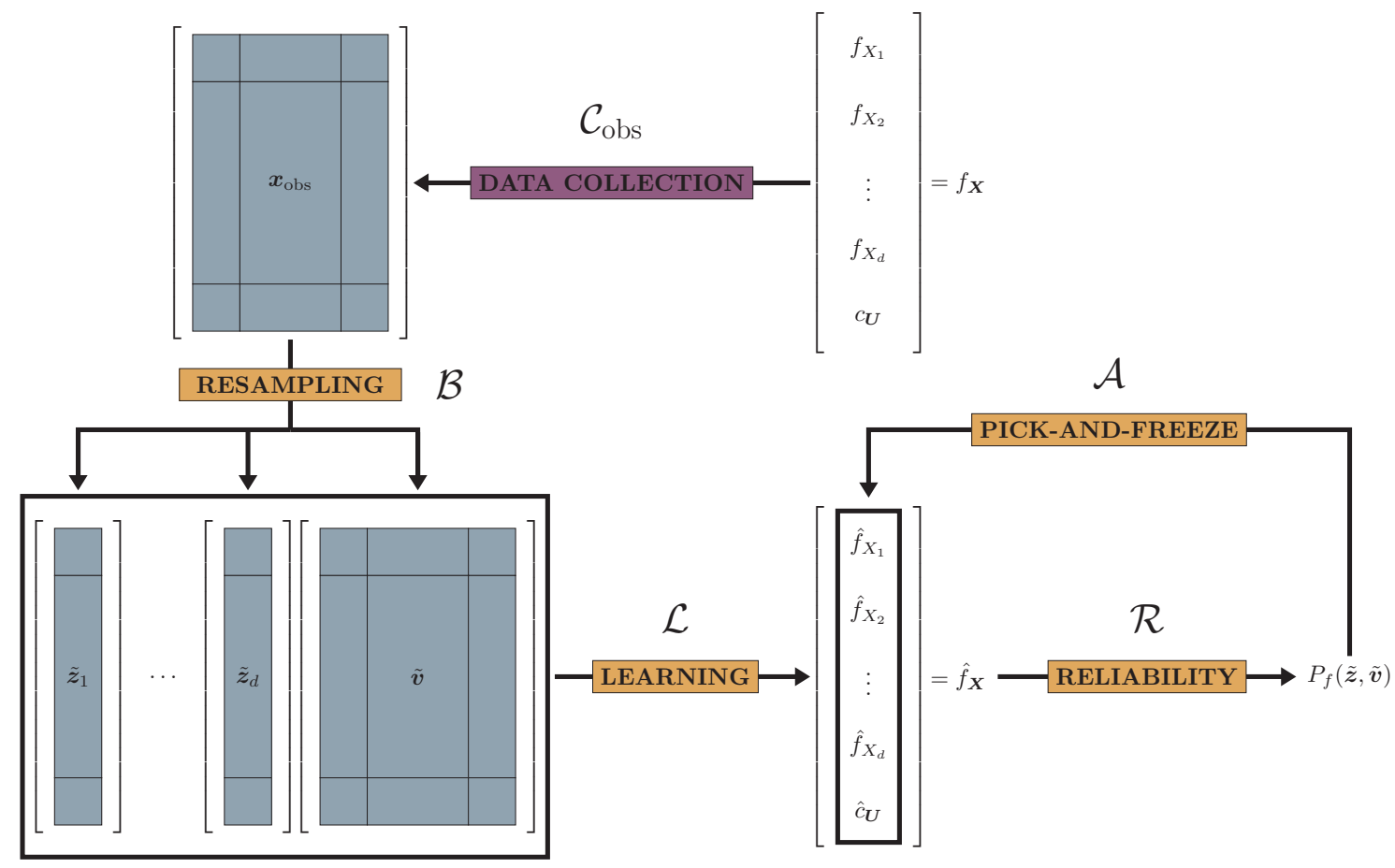

Figure 5: Illustration of all computational bricks when ROSA is performed with $\mathcal{R}$

Let us now examine how the "Pick-and-Freeze" estimation scheme works in practice. The main interest is to assess the computational budget that is required to derive all first-order Sobol indices. A few additional random objects need to be defined in order to adopt the "Pick-and-Freeze" viewpoint: 
$(\mathrm{PF} 1)\left(\tilde{\boldsymbol{Z}}^{A}, \tilde{\boldsymbol{V}}^{A}\right) \perp\left(\tilde{\boldsymbol{Z}}^{B}, \tilde{\boldsymbol{V}}^{B}\right)$ are distributed according to $\left(\hat{\mu}_{\boldsymbol{Z} \boldsymbol{V}}\left[\boldsymbol{x}_{\mathrm{obs}}\right]\right)^{\otimes N}$.

(PF2) Applying $\mathcal{L}$ on both collections yields $\left\{\hat{f}_{X_{1}}^{A}, \ldots, \hat{f}_{X_{d}}^{A}, \hat{c}_{U}^{A}\right\} \perp\left\{\hat{f}_{X_{1}}^{B}, \ldots, \hat{f}_{X_{d}}^{B}, \hat{c}_{\boldsymbol{U}}^{B}\right\}$.

(PF3) These collections are disassembled and inverted in order to construct a "Pick-and-Freeze" table $T_{A B}$ as illustrated in Figure 6. It is composed of $(d+2)$ recombined collections of functional components. With the exception of the first one, each row in $T_{A B}$ is obtained after freezing one component within $\left\{\hat{f}_{X_{1}}^{A}, \ldots, \hat{f}_{X_{d}}^{A}, \hat{c}_{\boldsymbol{U}}^{A}\right\}$ and picking all others among $\left\{\hat{f}_{X_{1}}^{B}, \ldots, \hat{f}_{X_{d}}^{B}, \hat{c}_{\boldsymbol{U}}^{B}\right\}$. The permutation operator leading to $T_{A B}$ is denoted by $\mathcal{P}$ in all what follows.

(PF4) In virtue of Sklar's theorem, each row in $T_{A B}$ may be reassembled into a joint input PDF. Reassembling is thus completed for all $(d+2)$ rows involved in $T_{A B}$.

(PF5) All corresponding failure probabilities are computed with $\mathcal{R}$.

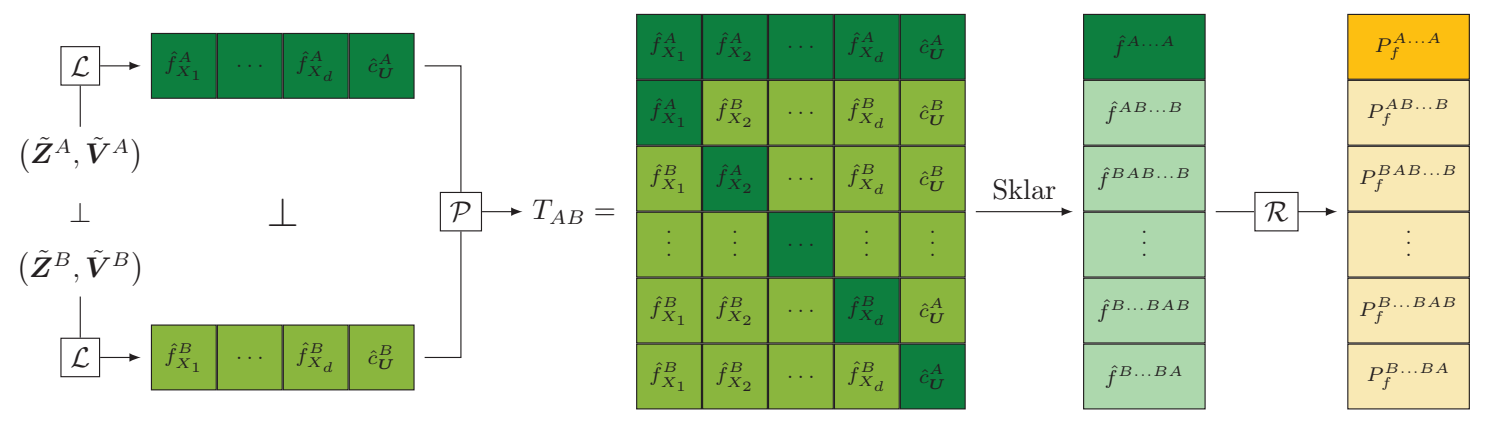

Figure 6: Application of the "Pick-and-Freeze" estimation scheme in the ROSA context

It must be clearly understood that the failure probabilities obtained at the end of the process (and represented on the right part of Figure 6) are random variables since they are expressed in terms of $\left(\tilde{\boldsymbol{Z}}^{A}, \tilde{\boldsymbol{V}}^{A}\right)$ and $\left(\tilde{\boldsymbol{Z}}^{B}, \tilde{\boldsymbol{V}}^{B}\right)$. In accordance with what was already said in Eq. (35), those failure probabilities allow to provide another expression of Sobol indices:

$$
\begin{gathered}
S_{1}^{\mathcal{B}}=\frac{\operatorname{Cov}\left(P_{f}^{A \ldots A}, P_{f}^{A B \ldots B}\right)}{\mathbb{V}\left(P_{f}^{A \ldots A}\right)} \quad \ldots \quad S_{d}^{\mathcal{B}}=\frac{\operatorname{Cov}\left(P_{f}^{A \ldots A}, P_{f}^{B \ldots B A B}\right)}{\mathbb{V}\left(P_{f}^{A} \ldots A\right)} \\
S_{\mathrm{cop}}^{\mathcal{B}}=\frac{\operatorname{Cov}\left(P_{f}^{A} \ldots A, P_{f}^{B \ldots B A}\right)}{\mathbb{V}\left(P_{f}^{A \ldots A}\right)} .
\end{gathered}
$$

The instructions listed from (PF1) to (PF5) stand for one iteration of the "Pick-and-Freeze" estimation scheme. If $\mathrm{M}$ iterations are carried out, $\mathcal{R}$ is run $M_{t}=(d+2) \times M$ times. Then, all Sobol indices can be estimated by replacing the theoretical variances and covariances involved in Eq. (49) by their empirical counterparts. 


\subsection{Application to a Gaussian linear model}

\subsubsection{Description}

A first use case is examined with a linear performance function $\phi(\cdot)$ and a Gaussian input random vector $\boldsymbol{X} \in \mathbb{R}^{3}$. The main merit of this example is to suit the initial assumption of this section, that is exact reliability assessment at negligible computational cost. The random output $Y$ is defined by:

$$
Y=\phi(\boldsymbol{X})=a_{1} X_{1}+a_{2} X_{2}+a_{3} X_{3}=\boldsymbol{a}^{\top} \boldsymbol{X} \quad \text { with } \quad \boldsymbol{a}=\left[a_{1}, a_{2}, a_{3}\right]^{\top}
$$

For its part, $\boldsymbol{X}$ is distributed as follows:

$$
\boldsymbol{X} \sim \mathcal{N}(\boldsymbol{m}, \boldsymbol{\Sigma}) \quad \text { with } \boldsymbol{m}=\left[\begin{array}{c}
m_{1} \\
m_{2} \\
m_{3}
\end{array}\right] \text { and } \boldsymbol{\Sigma}=\boldsymbol{\Gamma} \boldsymbol{\Gamma}^{\top}=\left[\begin{array}{ccc}
\sigma_{1}^{2} & \sigma_{12} & \sigma_{13} \\
\sigma_{12} & \sigma_{2}^{2} & \sigma_{23} \\
\sigma_{13} & \sigma_{23} & \sigma_{3}^{2}
\end{array}\right] \text {. }
$$

In this very simple case, the theoretical value of the failure probability $P_{f}$ can be derived through easy probabilistic calculations:

$$
P_{f}=\mathbb{P}\{Y>T\}=\mathbb{P}\left\{\boldsymbol{a}^{\top} \boldsymbol{X}>T\right\}=1-\Phi\left(\frac{T-m_{\boldsymbol{a}}}{\sigma_{\boldsymbol{a}}}\right) \text { with }\left\{\begin{array}{l}
m_{\boldsymbol{a}}=\boldsymbol{a}^{\top} \boldsymbol{m} \\
\sigma_{\boldsymbol{a}}^{2}=\boldsymbol{a}^{\top} \boldsymbol{\Sigma} \boldsymbol{a}
\end{array}\right.
$$

and $\Phi(\cdot)$ is the univariate standard normal CDF. Since $P_{f}$ is expected to be a rare-event probability, $T$ is understandably much larger than $m_{\boldsymbol{a}}$. In addition, as $\phi(\cdot)$ is linear and $\boldsymbol{X}$ is Gaussian, the first-order reliability method (FORM) is applicable and provides the exact failure probability $P_{f}$ after using the Nataf transform $\mathcal{T}$ to decorrelate $\boldsymbol{X}$ into a $d$-variate standard normal vector $\boldsymbol{W}$. Within this formalism [124, 125], the problem is entirely envisioned in the standard normal space. The limit state curve becomes $H(\boldsymbol{w})=T$ where $H=\phi \circ \mathcal{T}^{-1}$. Moreover, $P_{f}$ can be expressed in terms of both the Hasofer-Lindt reliability index $\beta$ and the most probable failure point (MPFP) which is denoted by $\boldsymbol{w}^{\star}$ (in the standard normal space) or $\boldsymbol{x}^{\star}$ (in the physical space):

$$
P_{f}=1-\Phi(\beta) \quad \text { with } \quad \beta:=\left\|\boldsymbol{w}^{\star}\right\|_{2} \quad \text { and } \quad \boldsymbol{w}^{\star}:=\underset{H(\boldsymbol{w})=T}{\arg \min }\|\boldsymbol{w}\|_{2}=\mathcal{T}\left(\boldsymbol{x}^{\star}\right)
$$

The numerical values assigned to the parameters of both $\phi(\cdot)$ and $f_{\boldsymbol{X}}$ are listed below:

- Black-box $\phi(\cdot)$ : $\quad a_{1}=a_{2}=2 ; a_{3}=1$

- Mean vector $\boldsymbol{m}$ : $\quad m_{1}=m_{2}=m_{3}=0$

- $\quad$ Covariance matrix $\boldsymbol{\Sigma}: \quad \sigma_{1}^{2}=\sigma_{2}^{2}=\sigma_{3}^{2}=1 ; \sigma_{12}=0.5 ; \sigma_{13}=\sigma_{23}=0.3$

With $T=11$, the theoretical value of failure probability is $P_{f}=2.53 \times 10^{-3}$. 


\subsubsection{Data-driven reliability assessment}

A dataset $\boldsymbol{x}_{\mathrm{obs}}$ is simulated according to $f_{\boldsymbol{X}}$ and numbers $N=300$ joint observations. The learning model $\mathcal{M}$ consists of a fully parametric Gaussian distribution. For a given collection of training sets $\left\{\tilde{\boldsymbol{z}}_{1}, \ldots, \tilde{\boldsymbol{z}}_{d}, \tilde{\boldsymbol{v}}\right\}$ produced by the bootstrap resampling technique $\mathcal{B}$ described earlier, a Gaussian margin is estimated from each $\tilde{\boldsymbol{z}}_{j}$ and a Gaussian copula is estimated from $\tilde{\boldsymbol{v}}$ :

$$
\hat{f}_{X_{j}}\left(\cdot \mid \mathcal{M}_{j}, \tilde{\boldsymbol{z}}_{j}\right)=\hat{f}_{\mathcal{N}}\left(\cdot \mid \hat{m}_{j}, \hat{\sigma}_{j}^{2}\right) \quad \text { and } \quad \hat{c}_{\boldsymbol{U}}\left(\cdot \mid \mathcal{M}_{\mathrm{cop}}, \tilde{\boldsymbol{v}}\right)=\hat{c}_{\mathcal{N}}(\cdot \mid \hat{\boldsymbol{\rho}})
$$

MLE is regarded as the best approach to fit all components. The joint reassembled PDF may be seen as a $d$-variate Gaussian PDF $\hat{f}_{\mathcal{N}}(\cdot \mid \hat{\boldsymbol{m}}, \hat{\boldsymbol{\Sigma}})$ such that:

$$
\hat{\boldsymbol{m}}=\left[\begin{array}{c}
\hat{m}_{1} \\
\hat{m}_{2} \\
\hat{m}_{3}
\end{array}\right] \text { and } \hat{\boldsymbol{\Sigma}}=\hat{\boldsymbol{\Gamma}} \hat{\boldsymbol{\Gamma}}^{\top}=\hat{\boldsymbol{\Delta}} \hat{\boldsymbol{\rho}} \hat{\boldsymbol{\Delta}} \quad \text { with } \quad \hat{\boldsymbol{\Delta}}=\left[\begin{array}{ccc}
\hat{\sigma}_{1} & 0 & 0 \\
0 & \hat{\sigma}_{2} & 0 \\
0 & 0 & \hat{\sigma}_{3}
\end{array}\right] \text {. }
$$

At the end of data-driven reliability assessment, one has:

$$
P_{f}\left(\boldsymbol{x}_{\mathrm{obs}}\right)=1-\Phi\left(\frac{T-\hat{m}_{\boldsymbol{a}}}{\hat{\sigma}_{\boldsymbol{a}}}\right) \text { with }\left\{\begin{array}{l}
\hat{m}_{\boldsymbol{a}}=\boldsymbol{a}^{\top} \hat{\boldsymbol{\mu}} \\
\hat{\sigma}_{\boldsymbol{a}}^{2}=\boldsymbol{a}^{\top} \hat{\boldsymbol{\Sigma}} \boldsymbol{a}
\end{array}\right.
$$

and the associated failure domain is specified in terms of the estimated Gaussian parameters:

$$
\hat{D}_{f}^{\mathcal{N}}=\left\{\boldsymbol{w} \in \mathbb{R}^{d}: \boldsymbol{a}^{\top}(\hat{\boldsymbol{\Gamma}} \boldsymbol{w}+\hat{\boldsymbol{\mu}})>T\right\}
$$

The uncertainty in reliability prediction only depends on whether or not $N$ amounts for a sufficient number of observations to expect an accurate reconstruction of PDF parameters.

\subsubsection{More about the theoretical values of Sobol indices}

The theoretical values of Sobol indices are those defined by Eq. (43). In this test case, where $\mathcal{M}$ is fully parametric, they may be rewritten in terms of estimated parameters:

$$
\begin{gathered}
\forall 1 \leq j \leq d, \quad S_{j}=\frac{\mathbb{V}\left(\mathbb{E}\left[P_{f}(\hat{\boldsymbol{m}}, \hat{\boldsymbol{\Sigma}}) \mid \hat{f}_{\mathcal{N}}\left(\cdot \mid \hat{m}_{j}, \hat{\sigma}_{j}\right)\right]\right)}{\mathbb{V}\left(P_{f}(\hat{\boldsymbol{m}}, \hat{\boldsymbol{\Sigma}})\right)}=\frac{\mathbb{V}\left(\mathbb{E}\left[P_{f}(\hat{\boldsymbol{m}}, \hat{\boldsymbol{\Sigma}}) \mid \hat{m}_{j}, \hat{\sigma}_{j}\right]\right)}{\mathbb{V}\left(P_{f}(\hat{\boldsymbol{m}}, \hat{\boldsymbol{\Sigma}})\right)}, \\
S_{\mathrm{cop}}=\frac{\mathbb{V}\left(\mathbb{E}\left[P_{f}(\hat{\boldsymbol{m}}, \hat{\boldsymbol{\Sigma}}) \mid \hat{c}_{\mathcal{N}}(\cdot \mid \hat{\boldsymbol{\rho}})\right]\right)}{\mathbb{V}\left(P_{f}(\hat{\boldsymbol{m}}, \hat{\boldsymbol{\Sigma}})\right)}=\frac{\mathbb{V}\left(\mathbb{E}\left[P_{f}(\hat{\boldsymbol{m}}, \hat{\boldsymbol{\Sigma}}) \mid \hat{\boldsymbol{\rho}}\right]\right)}{\mathbb{V}\left(P_{f}(\hat{\boldsymbol{m}}, \hat{\boldsymbol{\Sigma}})\right)},
\end{gathered}
$$

where $\hat{\boldsymbol{m}}$ and $\hat{\boldsymbol{\Sigma}}$ are estimated from $\left(\boldsymbol{Z}_{\text {obs }}, \boldsymbol{V}_{\text {obs }}\right) \sim\left(\mu_{\boldsymbol{Z} \boldsymbol{V}}\right)^{\otimes N}$ according to what is established in Eq. (54) and Eq. (55). Moreover, in light of Eq. (51), $\mathcal{G}_{\boldsymbol{Z}}$ and $\mathcal{G}_{\boldsymbol{V}}$ are the random generators associated with $\boldsymbol{Z} \sim f_{\mathcal{N}}\left(\cdot \mid \boldsymbol{m}, \boldsymbol{I}_{3}\right)$ and $\boldsymbol{V} \sim c_{\mathcal{N}}(\cdot \mid \boldsymbol{\rho})$. Because of the numerical values assigned to $f_{\boldsymbol{X}}$ and $\phi(\cdot)$ at the end of Section 3.5.1, similarities between $X_{1}$ and $X_{2}$ include: 
(Sym1) Sampling:

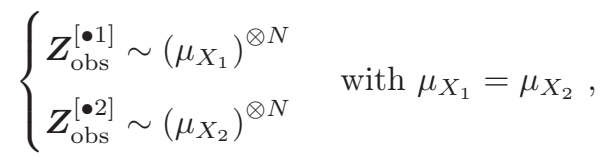

(Sym2) Learning: $\quad \mathcal{M}_{1}=\mathcal{M}_{2}$,

(Sym3) Dependence with $X_{3}: \quad\left\{\begin{array}{l}\left(\boldsymbol{V}_{\mathrm{obs}}^{[\bullet 1]}, \boldsymbol{V}_{\mathrm{obs}}^{[\bullet 3]}\right) \sim c_{\mathcal{N}}\left(\cdot \mid \rho_{13}\right) \\ \left(\boldsymbol{V}_{\mathrm{obs}}^{[\bullet 2]}, \boldsymbol{V}_{\mathrm{obs}}^{[\bullet \bullet 3]}\right) \sim c_{\mathcal{N}}\left(\cdot \mid \rho_{23}\right)\end{array} \quad\right.$ with $\rho_{13}=\rho_{23}$,

(Sym4) Black box: $\quad a_{1}=a_{2}=2$,

and it is straightforward to prove that all four similarities yield $S_{1}=S_{2}$. On the contrary, since (Sym3) and (Sym4) do not hold for $\left(X_{1}, X_{3}\right)$ and $\left(X_{2}, X_{3}\right)$, one has $S_{1} \neq S_{3}$ and $S_{2} \neq S_{3}$. Of course, the theoretical values $\left\{S_{1}, S_{2}, S_{3}, S_{\mathrm{cop}}\right\}$ cannot be derived in practice because the underlying distribution $\mu_{\boldsymbol{X}}$ is truly unknown. However, these indices may be helpful here in order to evaluate the accuracy of the collection $\left\{\hat{S}_{1}^{\mathcal{B}}, \hat{S}_{2}^{\mathcal{B}}, \hat{S}_{3}^{\mathcal{B}}, \hat{S}_{\text {cop }}^{\mathcal{B}}\right\}$ estimated by means of the resampling mechanism $\mathcal{B}$.

To this end, the theoretical indices are estimated through the "Pick-and-Freeze" method based on input training sets $\left\{\left(\boldsymbol{z}_{\text {obs }}^{(i)}, \boldsymbol{v}_{\text {obs }}^{(i)}\right)\right\}_{i=1}^{M}$ which are all simulated with $\mathcal{G}_{\boldsymbol{Z}}$ and $\mathcal{G}_{\boldsymbol{V}}$. The only hyperparameter that needs to be tuned is the number $M$ of iterations that is required to get low-variance estimators of Sobol indices. A trade-off must be found because the greater $M$, the more accurate Sobol indices, but the more there are failure probabilities to estimate. In the case where $N=300$, the results are presented in Figure 7 and it can be seen that all curves become stable for a pretty small number of replications, namely when $M \geq 10^{3}$. At this step, it can be seen that $\hat{S}_{1} \approx \hat{S}_{2}$ as it was expected in view of the above developments. Beyond this value, rankings are unchanged and further iterations strain the simulation budget without bringing any significant improvement. From now on, $M^{\star}=10^{3}$ is regarded as a recommendation for a proper tuning of the "Pick-and-Freeze" estimation scheme $\mathcal{A}$.

Sobol indices are estimated $N_{\text {rep }}=100$ times for $N \in\{50,100,300\}$ and the ensuing results are gathered in Table 1. A first point to make is the very low dependency shown by Sobol indices with respect to the sample size. The uncertainty carried by $P_{f}\left(\boldsymbol{Z}_{\mathrm{obs}}, \boldsymbol{V}_{\mathrm{obs}}\right)$ is always apportioned in the same way whatever is the number of observations used to fit the Gaussian margins and the Gaussian copula. For any given value of $N$, it is verified that $\hat{S}_{1}$ and $\hat{S}_{2}$ have very close values. As a consequence, and because of what was previously said about their equality, they receive the same ranking in Table 1. Moreover, $\hat{f}_{X_{1}}$ and $\hat{f}_{X_{2}}$ emerge as the most influential functional components as they both convey the largest share of uncertainty on $P_{f}\left(\boldsymbol{Z}_{\text {obs }}, \boldsymbol{V}_{\text {obs }}\right)$. Also noteworthy is that $\hat{S}_{\text {cop }}$ is somewhat smaller than $\hat{S}_{1}$ and $\hat{S}_{2}$ but much bigger then $\hat{S}_{3}$. It emphasizes the fact that copula estimation truly deserves attention when one attempts to monitor uncertainty on $P_{f}\left(\boldsymbol{Z}_{\text {obs }}, \boldsymbol{V}_{\text {obs }}\right)$. In this specific test case, it conveys almost as much uncertainty as $\hat{f}_{X_{1}}$ and $\hat{f}_{X_{2}}$ and much more than $\hat{f}_{X_{3}}$. 


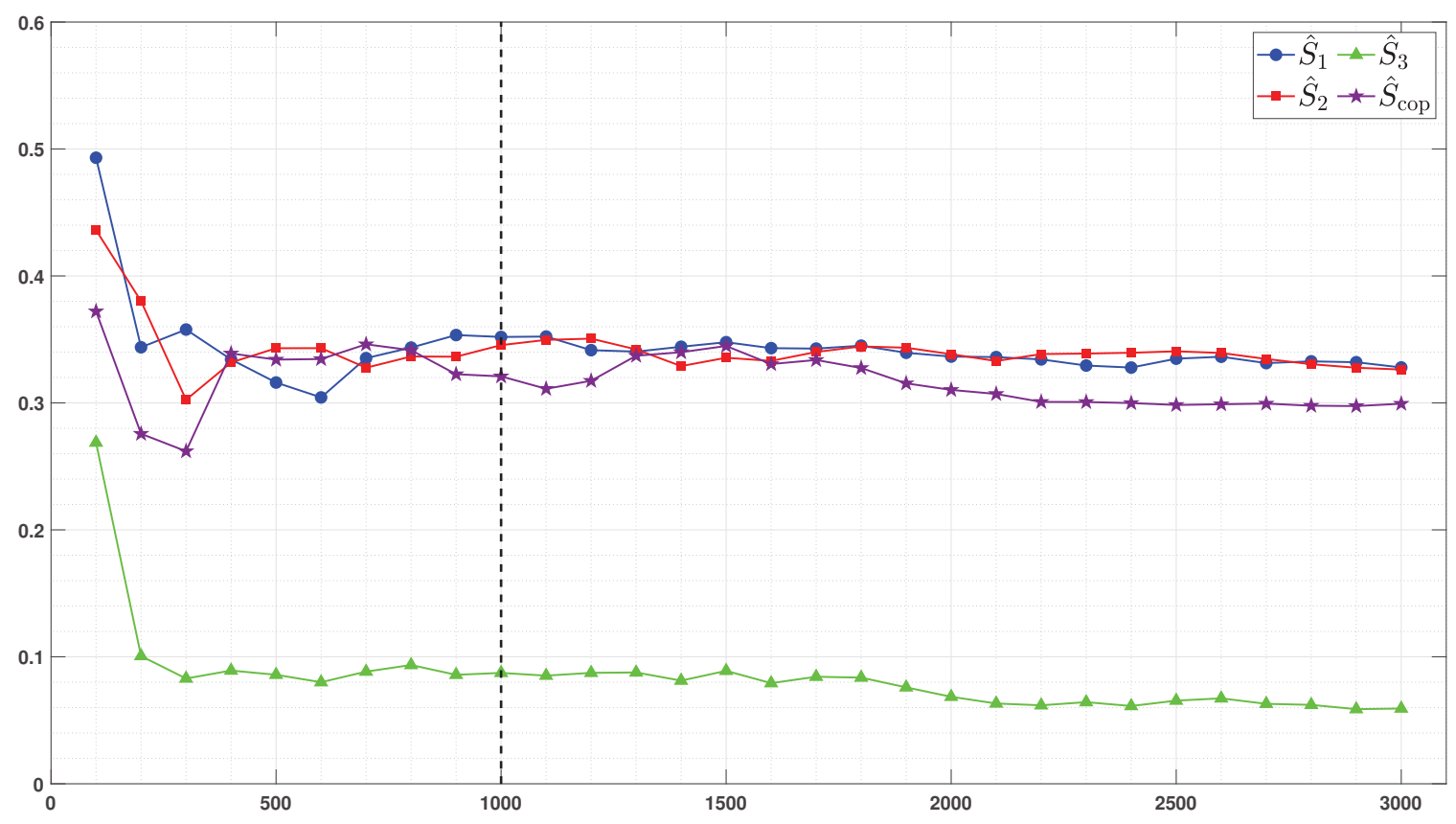

Figure 7: Convergence of estimates depending on the number $M$ of iterations used over the "Pick-and-Freeze".

\begin{tabular}{c|rcccc}
\hline ROSA with $\mathcal{G}_{\boldsymbol{Z}} / \mathcal{G}_{\boldsymbol{V}}:$ & $\hat{S}_{1}$ & $\hat{S}_{2}$ & $\hat{S}_{3}$ & $\hat{S}_{\text {cop }}$ \\
\hline \multirow{3}{*}{$N=300$} & mean : & $32.33 \%$ & $32.77 \%$ & $4.89 \%$ & $28.13 \%$ \\
& std : & $3.18 \%$ & $2.97 \%$ & $2.71 \%$ & $3.25 \%$ \\
& rank : & $(1)$ & $(1)$ & $(4)$ & $(3)$ \\
\hline \multirow{3}{*}{$N=100$} & mean : & $32.05 \%$ & $32.14 \%$ & $4.93 \%$ & $26.75 \%$ \\
& std : & $3.13 \%$ & $3.15 \%$ & $2.64 \%$ & $3.15 \%$ \\
& rank : & $(1)$ & $(1)$ & $(4)$ & $(3)$ \\
\hline \multirow{3}{*}{$N=50$} & mean : & $31.27 \%$ & $30.51 \%$ & $5.34 \%$ & $25.36 \%$ \\
& std : & $3.70 \%$ & $3.93 \%$ & $3.24 \%$ & $3.54 \%$ \\
& rank : & $(1)$ & $(1)$ & $(4)$ & $(3)$ \\
\hline
\end{tabular}

Table 1: Estimation of Sobol indices by means of the unknown generators $\mathcal{G}_{\boldsymbol{Z}}$ and $\mathcal{G}_{\boldsymbol{V}}$ 


\subsubsection{Estimation of Sobol indices from a small-sized dataset}

Let us now return to the data-driven ROSA problem. The theoretical generators $\mathcal{G}_{\boldsymbol{Z}}$ and $\mathcal{G}_{\boldsymbol{V}}$ are unknown and the available material consists of a single dataset $\boldsymbol{x}_{\text {obs }}$. Input training sets $\left\{\left(\tilde{\boldsymbol{z}}^{(i)}, \tilde{\boldsymbol{v}}^{(i)}\right)\right\}_{i=1}^{M}$ are simulated with the resampling mechanism $\mathcal{B}$ developed in Section 3.3 and relying on the empirical measure $\hat{\mu}_{\boldsymbol{Z} \boldsymbol{V}}\left[\boldsymbol{x}_{\mathrm{obs}}\right]$ defined in (46). In this context, it is feared that using the bootstrap induces a significant bias on the ROSA results. If so, the proposed algorithm becomes useless since no information about the theoretical indices might be extrapolated.

In order to address this issue, the ROSA algorithm is applied $N_{\text {rep }}=10$ times for three different datasets denoted by $\boldsymbol{x}_{\mathrm{obs}}^{(i)} \in \mathbb{R}^{N \times d}$ for $i \in\{1,2,3\}$ and $N=300$. The associated results may be found in the first level of Table 2. For all three datasets, the leading triplet consisting of $\left(\hat{S}_{1}, \hat{S}_{2}, \hat{S}_{\text {cop }}\right)$ is correctly identified and stands for more or less $90 \%$ of the uncertainty affecting $P_{f}(\tilde{\boldsymbol{Z}}, \tilde{\boldsymbol{V}})$. However, objections could be raised because the hierarchy among $\hat{f}_{X_{1}}, \hat{f}_{X_{2}}$ and $\hat{c}_{\boldsymbol{U}}$ evolves from one dataset to another :

$$
\begin{aligned}
& \boldsymbol{x}_{\mathrm{obs}}^{(1)} \Rightarrow \hat{S}_{\mathrm{cop}} \gg \hat{S}_{1}>\hat{S}_{2} \\
& \boldsymbol{x}_{\mathrm{obs}}^{(2)} \Rightarrow \hat{S}_{2}>\hat{S}_{\mathrm{cop}}>\hat{S}_{1} \\
& \boldsymbol{x}_{\mathrm{obs}}^{(3)} \Rightarrow \hat{S}_{1} \gg \hat{S}_{\mathrm{cop}}>\hat{S}_{2}
\end{aligned}
$$

This is naturally explained by the use of three different empirical measures. However, this lack of robustness in the rankings of the leading triplet of indices must be weighed against the fact that they have very close theoretical values. Actually, in view of Table 1, the most valuable information that needs to be gleaned from this test case is that the uncertainty on $P_{f}(\tilde{\boldsymbol{Z}}, \tilde{\boldsymbol{V}})$ stems from three equally influential sources. It must be noted that the data-driven ROSA procedure allows to draw a similar conclusion. From that point of view, the proposed algorithm fulfills the initial objective.

The same study is conducted for smaller datasets. In the second (resp. third) level of Table 2, $\boldsymbol{x}_{\mathrm{obs}}^{(i)}$ is made up with the first $N=100$ (resp. $N=50$ ) rows of the former version of $\boldsymbol{x}_{\mathrm{obs}}^{(i)}$. It can observed that the bias introduced over the data-driven ROSA procedure has increased. There is nothing surprising since the empirical probability measure $\hat{\mu}_{\boldsymbol{Z} \boldsymbol{V}}\left[\boldsymbol{x}_{\mathrm{obs}}\right]$ becomes a poor representation of the true underlying probability measure $\mu_{\boldsymbol{Z} \boldsymbol{V}}$ as $N$ decreases. For $N=100$, the ROSA based on $\boldsymbol{x}_{\mathrm{obs}}^{(2)}$ yields $\hat{S}_{\text {cop }}^{\mathcal{B}} \approx 40 \%$ instead of $S_{\text {cop }}=27 \%$. Likewise, when $N=50$, all three datasets lead to a heavy overestimation of the influence exerted by the copula fit. For this test case, as long as $N \geq N_{\lim }=100$, the proposed algorithm based on the resampling mechanism $\mathcal{B}$ works fine because it helps the user understanding what is the subset of functional components that most contribute to uncertainty in data-driven reliability assessment. However, when $N<N_{\lim }$, it becomes harder to regard the ROSA results as trustworthy piece of information for decision-making. The limit sample size $N_{\text {lim }}$ is not universal. All depends on the convergence of the empirical measure $\hat{\mu}_{\boldsymbol{Z} \boldsymbol{V}}\left[\boldsymbol{X}_{\mathrm{obs}}\right]$ with $\boldsymbol{X}_{\mathrm{obs}} \sim\left(\mu_{\boldsymbol{X}}\right)^{\otimes N}$. 
Any sample size $N$ becomes increasingly insufficient in higher dimensions. In particular, the empirical copula measure $\hat{\mu}_{\boldsymbol{V}}\left[\hat{\boldsymbol{U}}_{\text {obs }}\right]$ is gradually being affected by the curse of dimensionality. In such situations, the limit sample size is expected to be much larger than $N_{\lim }=100$.

Remark. In Table 2, all mean values and standard deviations are obtained after running $N_{\text {rep }}$ times the ROSA algorithm on the dataset under study. In this test case, as the reliability algorithm $\mathcal{R}$ is exact and does not introduce any extra noise on the distribution of $P_{f}(\tilde{\boldsymbol{Z}}, \tilde{\boldsymbol{V}})$, it should be clearly understood that uncertainty is only due to bootstrap resampling, that is to the fact that the sequence of all training sets $\left\{\left(\tilde{\boldsymbol{z}}^{(i)}, \tilde{\boldsymbol{v}}^{(i)}\right)\right\}_{i=1}^{M}$ changes from one repetition to another. Let $\left\{i_{k}\right\}_{k=1}^{M}$ be a bootstrap replication of $\{1, \ldots, M\}$. Another estimate of Sobol indices can be easily derived from the sequence of training sets $\left\{\left(\tilde{\boldsymbol{z}}^{\left(i_{k}\right)}, \tilde{\boldsymbol{v}}^{\left(i_{k}\right)}\right)\right\}_{k=1}^{M}$ because all the failure probabilities involved in the "Pick-and-Freeze" estimation scheme have already been computed. If repeating the process several times, one can obtain the mean values and the standard deviations without any additional call to $\mathcal{R}$.

\subsubsection{Validation through data assimilation}

Now, it is important to check whether the computed Sobol indices provide the expected answer in line with the objective of properly quantifying the epistemic uncertainties conveyed by the fit of both the margins and the copula. For a given dataset $\boldsymbol{x}_{\mathrm{obs}}$, the ROSA algorithm provides a set of Sobol indices denoted by $\left\{\hat{S}_{1}^{\mathcal{B}}, \hat{S}_{2}^{\mathcal{B}}, \hat{S}_{3}^{\mathcal{B}}, \hat{S}_{\mathrm{cop}}^{\mathcal{B}}\right\}$. A validation procedure could consist in performing a second ROSA after increasing the learning uncertainty of one poorly influential component or, conversely, after reducing the learning uncertainty of one highly influential component. The data-driven sensitivity indices are supposed to evolve accordingly, with a progress in the rankings for stimulated components and a collapse in the rankings for mitigated components. Let us imagine the fictitious situation where an additional database $\boldsymbol{x}_{\text {extra }}$ made up with $N_{2}$ observations from $\mu_{\boldsymbol{X}}$ is now available. For the sake of clarity, the sample size $N$ of $\boldsymbol{x}_{\mathrm{obs}}$ is denoted by $N_{1}$ in this subsection. The intent is to focus on a functional component $\hat{f}_{X_{j_{0}}}$ that has been identified as significantly influential after performing the first ROSA. The column $\boldsymbol{x}_{\text {extra }}^{\left[\bullet j_{0}\right]}$ is imported and acts as supplemental material for estimation. The state of knowledge about $\mu_{X_{j_{0}}}$ is thus enriched since $N_{1}+N_{2}$ observations are now supplied. Almost the same computational workflow is then applied to compute the second set of Sobol indices. A boostrap replication $\tilde{\boldsymbol{z}}_{j_{0}} \in \mathbb{R}^{N 1+N 2}$ is simulated from the enriched vector $\boldsymbol{x}_{\mathrm{obs}}^{\left[\bullet j_{0}\right]} \cup \boldsymbol{x}_{\mathrm{extra}}^{\left[\bullet j_{0}\right]}$. Other marginal training sets $\tilde{\boldsymbol{z}}_{j}\left(\right.$ with $\left.j \neq j_{0}\right)$ are directly replicated from $\boldsymbol{x}_{\mathrm{obs}}^{[\bullet j]}$. Besides, the copula training set $\tilde{\boldsymbol{v}}$ is still obtained after bootstrapping $\hat{\boldsymbol{u}}_{\text {obs }}$ but the rescaling step giving birth to $\hat{\boldsymbol{u}}_{\text {obs }}$ is slightly different (especially in the $j_{0}$-th column) in order to take into account the extra information brought by data assimilation. The reader is referred to Appendix A for a more comprehensive presentation of how the initial bootstrap mechanism is adapted. Appendix A.1 contains a pseudo-code framed box inspired from what has already been presented in Algorithm 3 while Appendix A.2 is dedicated to 


\begin{tabular}{|c|c|c|c|c|c|c|}
\hline & ROS & with $\mathcal{B}$ : & $\hat{S}_{1}$ & $\hat{S}_{2}$ & $\hat{S}_{3}$ & $\hat{S}_{\mathrm{cop}}$ \\
\hline \multirow{9}{*}{$N=300$} & & mean : & $28.26 \%$ & $26.56 \%$ & $5.03 \%$ & $37.49 \%$ \\
\hline & $\boldsymbol{x}_{\mathrm{obs}}^{(1)}$ & std : & $4.18 \%$ & $4.35 \%$ & $3.85 \%$ & $4.19 \%$ \\
\hline & & rank : & (2) & (3) & (4) & (1) \\
\hline & & mean : & $28.23 \%$ & $32.52 \%$ & $3.78 \%$ & $30.75 \%$ \\
\hline & $\boldsymbol{x}_{\mathrm{obs}}^{(2)}$ & std : & $3.82 \%$ & $3.77 \%$ & $3.62 \%$ & $2.84 \%$ \\
\hline & & rank : & $(3)$ & (1) & $(4)$ & $(2)$ \\
\hline & & mean : & $38.18 \%$ & $27.13 \%$ & $7.52 \%$ & $28.92 \%$ \\
\hline & $\boldsymbol{x}_{\mathrm{obs}}^{(3)}$ & std : & $2.34 \%$ & $3.88 \%$ & $3.41 \%$ & $3.91 \%$ \\
\hline & & rank: & $(1)$ & (3) & (4) & $(2)$ \\
\hline \multirow{9}{*}{$N=100$} & & mean : & $22.97 \%$ & $26.63 \%$ & $5.65 \%$ & $37.07 \%$ \\
\hline & $\boldsymbol{x}_{\mathrm{obs}}^{(1)}$ & std : & $4.04 \%$ & $4.30 \%$ & $2.26 \%$ & $3.98 \%$ \\
\hline & & rank: & $(3)$ & $(2)$ & $(4)$ & $(1)$ \\
\hline & & mean : & $17.03 \%$ & $33.65 \%$ & $5.14 \%$ & $39.95 \%$ \\
\hline & $\boldsymbol{x}_{\mathrm{obs}}^{(2)}$ & std : & $3.17 \%$ & $4.97 \%$ & $2.01 \%$ & $2.97 \%$ \\
\hline & & rank : & $(3)$ & $(2)$ & $(4)$ & $(1)$ \\
\hline & & mean : & $28.75 \%$ & $30.29 \%$ & $4.54 \%$ & $29.18 \%$ \\
\hline & $\boldsymbol{x}_{\mathrm{obs}}^{(3)}$ & std : & $3.23 \%$ & $4.33 \%$ & $3.12 \%$ & $3.20 \%$ \\
\hline & & rank : & $(3)$ & (1) & (4) & $(2)$ \\
\hline \multirow{9}{*}{$N=50$} & & mean : & $25.48 \%$ & $19.28 \%$ & $5.81 \%$ & $37.34 \%$ \\
\hline & $\boldsymbol{x}_{\mathrm{obs}}^{(1)}$ & std : & $3.30 \%$ & $4.88 \%$ & $3.51 \%$ & $3.06 \%$ \\
\hline & & rank : & $(2)$ & $(3)$ & $(4)$ & $(1)$ \\
\hline & & mean : & $16.66 \%$ & $36.42 \%$ & $2.88 \%$ & $34.59 \%$ \\
\hline & $\boldsymbol{x}_{\mathrm{obs}}^{(2)}$ & std : & $2.22 \%$ & $2.01 \%$ & $2.22 \%$ & $3.79 \%$ \\
\hline & & rank : & $(3)$ & $(1)$ & $(4)$ & $(2)$ \\
\hline & & mean : & $23.56 \%$ & $20.85 \%$ & $6.95 \%$ & $38.35 \%$ \\
\hline & $\boldsymbol{x}_{\mathrm{obs}}^{(3)}$ & std : & $1.92 \%$ & $3.77 \%$ & $2.29 \%$ & $4.73 \%$ \\
\hline & & rank: & $(2)$ & (3) & $(4)$ & $(1)$ \\
\hline
\end{tabular}

Table 2: Estimation of Sobol indices from three Gaussian datasets with the ROSA procedure 
visualizing the necessary matrix manipulations. The whole Appendix A is presented in the specific case where additional data are collected for $X_{1}$ but everything is similar for other input variables.

Table 3 presents the results obtained after performing a second ROSA for three different data assimilation scenarios. The top level recalls the results previously obtained with $\boldsymbol{x}_{\mathrm{obs}}^{(1)}$ in Table 2. The three levels below show the results delivered by the second ROSA after providing additional data to $X_{1}, X_{2}$ or $X_{3}$. In the two first cases, data assimilation makes the targeted component become the less influential source of learning uncertainty. This phenomenon is consistent with what the ROSA algorithm was designed for. Furthermore, when new observations are provided to $X_{3}$, no change is observed in the rankings. It is rather natural since $\hat{f}_{X_{3}}$ has already emerged as the less influential component in the first ROSA. Incorporating even more data in the estimation of $f_{X_{3}}$ reduces even more the impact of $\hat{f}_{X_{3}}$ on the uncertainty of reliability assessment. Once again, this sticks to what Sobol indices are supposed to quantify.

\section{Extension to stochastic reliability algorithms}

In the previous section, in order to measure the amount of epistemic uncertainty conveyed on the failure probability by the margins and the copula, an algorithm was implemented to compute GSA indicators. However, the proposed method is only valid if reliability assessment $\mathcal{R}$ can be implemented exactly and without any computational effort. Now, let us go back to a more realistic situation where rare-event probability estimation is performed with simulation algorithms. Contrary to what is shown in Section 3.5 where having access to the analytical expression of $\phi(\cdot)$ enables to set up an adhoc exact formula for $P_{f}$, the internal workings of $\phi(\cdot)$ are unknown in most cases. As a result, the output PDF $f_{Y}$ has to be assessed in one way or another, especially in the upper tail where the failure event occurs. The simulation budget often consists of a limited number of evaluations of $\phi(\cdot)$, which is largely insufficient to retrieve $f_{Y}$ on its whole definition range. Rare-event probability estimation algorithms based on adaptive resampling techniques have to deployed not to overrun the allotted simulation budget. As a consequence, for a given learnt distribution $\hat{f}_{\boldsymbol{X}}$, reliability estimation is now predicated on a stochastic algorithm $\mathcal{R}_{w}$ representing one of the many working methods in this field [2].

These algorithms are described as stochastic because, even for a specified input PDF $\hat{f}_{\boldsymbol{X}}$, the estimated failure probability $\hat{P}_{f}=\mathcal{R}_{w}\left(\hat{f}_{\boldsymbol{X}}\right)$ is a random variable depending on the simulated particles. For the sake of consistency, data-driven reliability assessment is now denoted by $\mathcal{D}_{w}$ instead of $\mathcal{D}$ :

$$
\hat{P}_{f}\left(\boldsymbol{x}_{\mathrm{obs}}\right)=\mathcal{D}_{w}\left(\boldsymbol{x}_{\mathrm{obs}}\right)=\left[\mathcal{R}_{w} \circ \mathcal{L}\right]\left(\boldsymbol{x}_{\mathrm{obs}}\right)
$$

Likewise, the input-output mapping $\Psi$ between the components of the estimated PDF $\hat{f}_{\boldsymbol{X}}\left(\cdot \mid \boldsymbol{X}_{\text {obs }}\right)$ 


\begin{tabular}{|c|c|c|c|c|c|c|c|}
\hline \multicolumn{4}{|c|}{ ROSA with $\mathcal{B}$ : } & $\hat{S}_{1}^{\mathcal{B}}$ & $\hat{S}_{2}^{\mathcal{B}}$ & $\hat{S}_{3}^{\mathcal{B}}$ & $\hat{S}_{\mathrm{cop}}^{\mathcal{B}}$ \\
\hline & \multirow{3}{*}{$\boldsymbol{x}_{\mathrm{obs}}=\boldsymbol{x}_{\mathrm{obs}}^{(1)}$} & \multirow{3}{*}{$N_{1}=300$} & mean : & $28.26 \%$ & $26.56 \%$ & $5.03 \%$ & $37.49 \%$ \\
\hline & & & std : & $4.18 \%$ & $4.35 \%$ & $3.85 \%$ & $4.19 \%$ \\
\hline & & & rank : & $(2)$ & (3) & $(4)$ & (1) \\
\hline & & \multicolumn{2}{|c|}{ ROSA with $\mathcal{B}_{1}$ : } & $\hat{S}_{1}^{\mathcal{B}_{1}}$ & $\hat{S}_{2}^{\mathcal{B}_{1}}$ & $\hat{S}_{3}^{\mathcal{B}_{1}}$ & $\hat{S}_{\mathrm{cop}}^{\mathcal{B}_{1}}$ \\
\hline \multirow{6}{*}{ 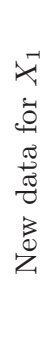 } & \multirow{6}{*}{$\boldsymbol{x}_{\mathrm{obs}} \cup \boldsymbol{x}_{\text {extra }}^{[\bullet 1]}$} & \multirow{3}{*}{$N_{2}=500$} & mean : & $14.65 \%$ & $30.94 \%$ & $4.91 \%$ & $44.99 \%$ \\
\hline & & & std : & $2.82 \%$ & $3.82 \%$ & $3.58 \%$ & $3.69 \%$ \\
\hline & & & rank : & (3) & $(2)$ & (4) & $(1)$ \\
\hline & & \multirow{3}{*}{$N_{2}=10^{3}$} & mean : & $12.68 \%$ & $32.72 \%$ & $7.08 \%$ & $49.37 \%$ \\
\hline & & & std : & $2.69 \%$ & $3.58 \%$ & $3.39 \%$ & $2.95 \%$ \\
\hline & & & rank : & $(3)$ & $(2)$ & $(4)$ & $(1)$ \\
\hline \multirow{7}{*}{ 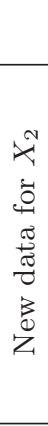 } & & \multicolumn{2}{|c|}{ ROSA with $\mathcal{B}_{2}$ : } & $\hat{S}_{1}^{\mathcal{B}_{2}}$ & $\hat{S}_{2}^{\mathcal{B}_{2}}$ & $\hat{S}_{3}^{\mathcal{B}_{2}}$ & $\hat{S}_{\mathrm{cop}}^{\mathcal{B}_{2}}$ \\
\hline & \multirow{6}{*}{$\boldsymbol{x}_{\mathrm{obs}} \cup \boldsymbol{x}_{\mathrm{extra}}^{[\bullet 2]}$} & \multirow{3}{*}{$N_{2}=500$} & mean : & $31.51 \%$ & $14.26 \%$ & $5.31 \%$ & $44.68 \%$ \\
\hline & & & std : & $3.07 \%$ & $3.23 \%$ & $1.80 \%$ & $2.06 \%$ \\
\hline & & & rank : & $(2)$ & $(3)$ & $(4)$ & $(1)$ \\
\hline & & \multirow{3}{*}{$N_{2}=10^{3}$} & mean : & $33.61 \%$ & $10.37 \%$ & $6.42 \%$ & $49.58 \%$ \\
\hline & & & std : & $3.52 \%$ & $2.09 \%$ & $2.43 \%$ & $3.40 \%$ \\
\hline & & & rank : & $(2)$ & $(3)$ & $(4)$ & $(1)$ \\
\hline \multirow{7}{*}{ 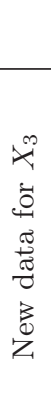 } & & \multicolumn{2}{|c|}{ ROSA with $\mathcal{B}_{3}$ : } & $\hat{S}_{1}^{\mathcal{B}_{3}}$ & $\hat{S}_{2}^{\mathcal{B}_{3}}$ & $\hat{S}_{3}^{\mathcal{B}_{3}}$ & $\hat{S}_{\mathrm{cop}}^{\mathcal{B}_{3}}$ \\
\hline & \multirow{6}{*}{$\boldsymbol{x}_{\text {obs }} \cup \boldsymbol{x}_{\text {extra }}^{[\bullet 3]}$} & \multirow{3}{*}{$N_{2}=500$} & mean : & $28.79 \%$ & $29.50 \%$ & $4.01 \%$ & $40.94 \%$ \\
\hline & & & std : & $3.51 \%$ & $2.36 \%$ & $2.41 \%$ & $2.11 \%$ \\
\hline & & & rank : & $(3)$ & $(2)$ & $(4)$ & $(1)$ \\
\hline & & \multirow{3}{*}{$N_{2}=10^{3}$} & mean : & $29.27 \%$ & $29.79 \%$ & $3.79 \%$ & $39.48 \%$ \\
\hline & & & std : & $3.33 \%$ & $3.29 \%$ & $2.74 \%$ & $2.32 \%$ \\
\hline & & & rank: & $(3)$ & & $(4)$ & $(1)$ \\
\hline
\end{tabular}

Table 3: Qualitative validation of the ROSA results obtained from $\boldsymbol{x}_{\mathrm{obs}}$ thanks to data assimilation 
and the estimated failure probability $\hat{P}_{f}\left(\boldsymbol{X}_{\text {obs }}\right)$ become $\Psi_{w}$ to comply with the notation changes:

$$
\begin{aligned}
& \Psi_{w}: \quad \mathbb{L}^{2}(\mathbb{R}) \times \ldots \times \mathbb{L}^{2}(\mathbb{R}) \times \mathbb{L}^{2}\left(\mathcal{H}_{d}\right) \quad \longrightarrow \quad[0,1] \\
& \mathcal{F}:=\bigcup_{j=1}^{d}\left\{\hat{f}_{X_{j}}\left(\cdot \mid \boldsymbol{X}_{\mathrm{obs}}\right)\right\} \cup\left\{\hat{c}_{\boldsymbol{U}}\left(\cdot \mid \boldsymbol{X}_{\mathrm{obs}}\right)\right\} \longmapsto \hat{P}_{f}\left(\boldsymbol{X}_{\mathrm{obs}}\right)
\end{aligned}
$$

Two major difficulties arise from this situation. Firstly, the use of stochastic algorithms induces an additional uncertainty in reliability assessment. Despite the appearance of this unwanted extra uncertainty, the same computational workflow can still be applied to estimate Sobol indices. However, the noise introduced by $\mathcal{R}_{w}$ may cause instability in the ROSA results. For a fixed dataset $\boldsymbol{x}_{\text {obs }}$, it should be avoided that several runs of the same algorithm do not end up with substantially equivalent results. For this reason, it must be checked that Sobol indices retain the same statistical properties, especially in terms of dispersion. Secondly, if $\mathcal{R}_{w}$ is embedded within the "Pick-and-Freeze" estimation scheme, the overall computational burden can no longer be afforded. To circumvent this pitfall, it will be shown that the mathematical structure of importance sampling opens up possibilities in order to perform ROSA at a reasonable computational cost.

\subsection{Variance reduction with importance sampling}

In reliability applications, the CMC estimator defined in Eq. (5) is most often inappropriate because its coefficient of variation requires too many black-box evaluations before being lowered to an acceptable level. Variance reduction can be achieved after replacing $f_{\boldsymbol{X}}$ by an auxiliary PDF denoted by $h$ [126]. Since its early introduction in the reliability community [127, 128], importance sampling (IS) is a widely used reference technique for reliability analysis. One may write that:

$$
P_{f}=\mathbb{E}_{f_{\boldsymbol{X}}}\left[\mathbb{1}_{D_{f}}(\boldsymbol{X})\right]=\mathbb{E}_{h}\left[\mathbb{1}_{D_{f}}(\boldsymbol{X}) \frac{f_{\boldsymbol{X}}(\boldsymbol{X})}{h(\boldsymbol{X})}\right],
$$

and the resulting IS estimator is then given by:

$$
\hat{P}_{f}^{\text {IS }}=\frac{1}{N_{s}} \sum_{i=1}^{N_{s}} \mathbb{1}_{\left\{Y^{(i)}>T\right\}} \frac{f_{\boldsymbol{X}}\left(\boldsymbol{X}^{(i)}\right)}{h\left(\boldsymbol{X}^{(i)}\right)} \quad \text { with }\left\{\begin{array}{l}
\boldsymbol{X}^{(i)} \stackrel{\text { i.i.d. }}{\sim} h \\
Y^{(i)}=\phi\left(\boldsymbol{X}^{(i)}\right)
\end{array} .\right.
$$

It is unbiased as long as $h$ meets the following condition:

$$
\left(D_{f} \cap\left\{\boldsymbol{x} \in \mathbb{R}^{d}: f_{\boldsymbol{X}}(\boldsymbol{x})>0\right\}\right) \subseteq\left\{\boldsymbol{x} \in \mathbb{R}^{d}: h(\boldsymbol{x})>0\right\},
$$

but this does not necessarily enable variance reduction. For any auxiliary PDF $h$, one has:

$$
\mathbb{V}\left(\hat{P}_{f}^{\mathrm{IS}}\right)=\frac{1}{N} \mathbb{V}_{h}\left(\mathbb{1}_{\{\phi(\boldsymbol{X})>T\}} \frac{f_{\boldsymbol{X}}(\boldsymbol{X})}{h(\boldsymbol{X})}\right),
$$


and it can be easily proved [1] that the variance is minimized and becomes equal to zero when the inner quantity is almost surely a constant scalar. As a consequence, the optimal sampling PDF is obtained if $h$ is the conditional PDF of $\boldsymbol{X}$ with respect to the failure domain:

$$
h_{\mathrm{opt}}(\boldsymbol{x})=\frac{\mathbb{1}_{D_{f}}(\boldsymbol{x}) f_{\boldsymbol{X}}(x)}{P_{f}} .
$$

Since the normalization constant coincides with the quantity of interest $P_{f}$, the optimal PDF cannot be used unaltered. A problem for practitioners is to find a "suboptimal" candidate $\hat{h}_{\text {opt }}$ that might be able to replace $h_{\mathrm{opt}}$. In the literature, a considerable amount of highly relevant research works ambition to provide a generic methdology to construct $\hat{h}_{\text {opt }}$. A first solution is to look for the best approximation within a specific class of distributions [129, 130, 131]. For a given parametric model, a suboptimal auxiliary PDF $\boldsymbol{x} \longmapsto h\left(\boldsymbol{x} \mid \boldsymbol{\eta}^{\star}\right)$ can be identified with the cross-entropy algorithm [132]. However, there are situations where making a parametric assumption may be seriously misleading. To break the deadlock, learning $h_{\text {opt }}$ with a nonparametric method can be contemplated with a large range of kernel-based techniques [133, 134, 135]. It is shown in [136] that the nonparametric approach allows for a faster convergence to the target failure probability. A variant of the initial algorithm based on weighted Gaussian kernel is proposed in [137] and will be used on several occasions thereafter. However, it must be underlined that all what is stated from now on holds for any IS recipe because the key element of the computational strategy only rests on having access to an efficient auxiliary distribution $\hat{h}_{\mathrm{opt}}$.

\subsection{Computational gains with the "inverse-reverse" trick}

As it was explained in Section 3.4, the proposed ROSA method relies on the estimation of $M_{t}$ failure probabilities by means of $\mathcal{R}_{w}$. Let $\left\{\hat{g}^{(i)}\right\}_{i=1}^{M_{t}}$ be the sequence collecting all input PDFs generated by the "Pick-and-Freeze" estimation scheme. For each reassembled joint distribution $\hat{g}^{(i)}$, the optimal auxiliary distribution $h_{\mathrm{opt}}^{(i)}$ is:

$$
h_{\mathrm{opt}}^{(i)}(\boldsymbol{x})=\frac{\mathbb{1}_{D_{f}}(\boldsymbol{x}) \hat{g}^{(i)}(\boldsymbol{x})}{P_{f}^{(i)}} \quad \text { with } \quad P_{f}^{(i)}=\mathbb{E}_{\hat{g}^{(i)}}\left[\mathbb{1}_{D_{f}}(\boldsymbol{X})\right]
$$

After constructing an auxiliary distribution $\hat{h}_{\mathrm{opt}}^{(i)}$, an IS estimator for $P_{f}^{(i)}$ comes naturally:

$$
\hat{P}_{f}^{(i)}=\frac{1}{N_{s}} \sum_{k=1}^{N_{s}} \mathbb{1}_{\left\{Y^{(k)}>T\right\}} \frac{\hat{g}^{(i)}\left(\boldsymbol{X}^{(k)}\right)}{\hat{h}_{\mathrm{opt}}^{(i)}\left(\boldsymbol{X}^{(k)}\right)} \quad \text { with }\left\{\begin{array}{l}
\boldsymbol{X}^{(k)} \stackrel{\text { i.i.d }}{\sim} \hat{h}_{\mathrm{opt}}^{(i)} \\
Y^{(k)}=\phi\left(\boldsymbol{X}^{(k)}\right)
\end{array} .\right.
$$

IS is a much better practice than CMC but it is still insufficient from a computational standpoint especially if one plans to repeat sequentially the reliability algorithm $\mathcal{R}_{w}$ in order to perform ROSA. The algorithm resulting from parallel CMC runs is illustrated on Figure 8a. The analogous situation 
in the event of parallel IS runs is reprensented on Figure 8b. In this situation, the total number $N_{\text {tot }}$ of calls to $\phi(\cdot)$ is given by:

$$
N_{\text {tot }}=M_{t} \times N_{\text {IS }},
$$

where $N_{\mathrm{IS}}$ is the number of calls to $\phi(\cdot)$ at each IS run. If $\hat{h}_{\mathrm{opt}}$ is provided, $N_{\mathrm{IS}}=N_{s}$ since $\phi(\cdot)$ is only used to compute the outputs $Y^{(k)}$ associated to the input particles $\boldsymbol{X}^{(k)}$. On the contrary, when $\hat{h}_{\text {opt }}$ is unknown, an adaptive method has to be used. It implies several resampling steps in order to displace an initial set of input particles towards the failure region $D_{f}$. At the end of this sequential exploration, the user is delivered both $\hat{h}_{\mathrm{opt}}$ and the input-output samples $\left(\boldsymbol{X}^{(k)}, Y^{(k)}\right)$ produced by the last resampling step. In this situation, $N_{\text {IS }}=N_{\text {iter }} \times N_{s}$ with $N_{\text {iter }}$ being the number of iterations before the stopping criterion is enabled. A more detailed expression of Eq. (68) follows:

$$
N_{\text {tot }}=(d+2) \times M \times N_{\text {iter }} \times N_{s} .
$$

In order to secure a small coefficient of variation, it is often decided to take $N_{s} \geq 10^{3}$. Remembering that $M \geq 10^{3}$, the total number of calls to $\phi(\cdot)$ is such that $N_{\text {tot }} \geq K \times 10^{6}$ where $K$ is a multiplying factor that inflates when either $d$ increases or $P_{f}$ decreases. So many calls to $\phi(\cdot)$ cannot be afforded. As a consequence, running an IS algorithm for every input PDF $\hat{g}^{(i)}$ is not a computationally viable solution. A remedy is provided by the so-called "inverse-reverse" trick.

Let us imagine that an IS algorithm is run once for a given input distribution $\hat{g}^{(1)}$. This produces an auxiliary PDF $\hat{h}_{\mathrm{opt}}^{(1)}$ along with $N$ independent input samples $\boldsymbol{X}^{(k)} \stackrel{\text { i.i.i. }}{\sim} \hat{h}_{\mathrm{opt}}^{(1)}$ and their associated outputs $Y^{(k)}$. It could be very convenient to recycle this material in order to estimated the failure probabilities associated to other input PDFs. In this regard, one can notice that :

$$
P_{f}^{(i)}=\mathbb{E}_{\hat{g}^{(i)}}\left[\mathbb{1}_{D_{f}}(\boldsymbol{X})\right]=\mathbb{E}_{\hat{h}_{\mathrm{opt}}^{(1)}}\left[\mathbb{1}_{D_{f}}(\boldsymbol{X}) \frac{\hat{g}^{(i)}(\boldsymbol{X})}{\hat{h}_{\mathrm{opt}}^{(1)}(\boldsymbol{X})}\right],
$$

and another IS estimator of $P_{f}^{(i)}$ follows:

$$
\left.\hat{P}_{f}^{(i)}=\frac{1}{N_{s}} \sum_{k=1}^{N_{s}} \mathbb{1}_{\left\{Y^{(k)}>T\right\}}\right\} \frac{\hat{g}^{(i)}\left(\boldsymbol{X}^{(k)}\right)}{\hat{h}_{\mathrm{opt}}^{(1)}\left(\boldsymbol{X}^{(k)}\right)} \quad \text { with }\left\{\begin{array}{l}
\boldsymbol{X}^{(k)} \stackrel{\text { i.i.j. }}{\sim} \hat{h}_{\mathrm{opt}}^{(1)} \\
Y^{(k)}=\phi\left(\boldsymbol{X}^{(k)}\right)
\end{array} .\right.
$$

This one is not suboptimal but it offers the advantage of being computable with all the material delivered by the first IS run. The expected value of $\hat{P}_{f}^{(i)}$ stays equal to $P_{f}^{(i)}$ but the variance is not at the lowest achievable level, which may arouse skepticism on whether this method is really accurate. This trick has already been used in previous works $[138,139]$ to update a quantity of interest or to construct a confidence interval. The numerical efficiency of the modified IS estimator greatly depends on how close $\hat{h}_{\mathrm{opt}}^{(1)}$ and $\hat{h}_{\mathrm{opt}}^{(i)}$ are. Figure $8 \mathrm{c}$ makes it clear how the "inverse-reverse" trick allows to carry 
out ROSA within reasonable CPU time. Seeing that only one IS run is accepted, the total number of calls to $\phi(\cdot)$ is now simply limited to:

$$
N_{\text {tot }}=N_{\text {iter }} \times N_{s} .
$$

\subsection{Enhanced robustness with multiple importance sampling}

Whether the "inverse-reverse trick" enables worthwhile computational gains, the main drawback is that it also contributes to deteriorate the robustness of the ROSA procedure. In fact, the systematic use of $\hat{h}_{\mathrm{opt}}^{(1)}$ for all IS estimates increases the risk of encountering cases where the estimator $\hat{P}_{f}^{(i)}$ defined in Eq. (71) has a large variance. To overcome this pitfall, a natural idea is to involve a small number of auxiliary PDFs instead of only one. For instance, if the IS algorithm is run for the $n$ first PDFs $\hat{g}^{(i)}$, one may write:

$$
\forall 1 \leq i \leq n, \quad \hat{P}_{f}^{(i)}=\frac{1}{N_{s}} \sum_{k=1}^{N_{s}} \mathbb{1}_{\left\{Y_{i}^{(k)}>T\right\}} \frac{\hat{g}^{(i)}\left(\boldsymbol{X}_{i}^{(k)}\right)}{\hat{h}_{\mathrm{opt}}^{(i)}\left(\boldsymbol{X}_{i}^{(k)}\right)} \quad \text { with }\left\{\begin{array}{l}
\boldsymbol{X}_{i}^{(k)} \stackrel{\text { i.i.d }}{\sim} \hat{h}_{\mathrm{opt}}^{(i)} \\
Y_{i}^{(k)}=\phi\left(\boldsymbol{X}_{i}^{(k)}\right)
\end{array} .\right.
$$

The input samples simulated with $\hat{h}_{\mathrm{opt}}^{(i)}$ are now denoted by $\boldsymbol{X}_{i}^{(k)}$ so as to prevent any confusion. The associated output samples become $Y_{i}^{(k)}$ accordingly. The issue is then to understand how to combine all auxiliary PDFs within a low-variance estimator that could be applied for any other distribution $\hat{g}$ involved in the ROSA procedure. Multiple importance sampling (MIS) consists in introducing a weighting scheme to improve robustness $[35,140]$ and leads to consider the following estimator:

$$
\hat{P}_{f}^{w}=\frac{1}{N_{s}} \sum_{k=1}^{N_{s}} \sum_{i=1}^{n} w_{i}\left(\boldsymbol{X}_{i}^{(k)}\right) \mathbb{1}_{\left\{Y_{i}^{(k)}>T\right\}} \frac{\hat{g}\left(\boldsymbol{X}_{i}^{(k)}\right)}{\hat{h}_{\mathrm{opt}}^{(i)}\left(\boldsymbol{X}_{i}^{(k)}\right)} \quad \text { with }\left\{\begin{array}{l}
\boldsymbol{X}_{i}^{(k)} \stackrel{\text { i.i.j. }}{\sim} \hat{h}_{\mathrm{opt}}^{(i)} \\
Y_{i}^{(k)}=\phi\left(\boldsymbol{X}_{i}^{(k)}\right)
\end{array} .\right.
$$

The weighting function $w_{i}(\cdot)$ accounts for the influence that must be given to samples coming from the auxiliary distribution $\hat{h}_{\mathrm{opt}}^{(i)}$. $\hat{P}_{f}^{w}$ remains unbiased only if the weighting functions $w_{i}(\cdot)$ are a partition of unity, that is:

$$
\forall \boldsymbol{x} \in \mathbb{R}^{d}, \quad \sum_{i=1}^{n} w_{i}(\boldsymbol{x})=1,
$$

Among the proposal for weighting functions, the balance heuristic $(\mathrm{BH})$ is one of the most studied but many others have also been used [141]. For BH, the idea is to compute the ratio between $\hat{h}_{\mathrm{opt}}^{(i)}$ and the sum of all auxiliary PDFs:

$$
w_{i}^{\mathrm{BH}}(\boldsymbol{x})=\frac{\hat{h}_{\mathrm{opt}}^{(i)}(\boldsymbol{x})}{\sum_{l=1}^{n} \hat{h}_{\mathrm{opt}}^{(l)}(\boldsymbol{x})} .
$$

The MIS estimator defined in Eq. (74) then becomes:

$$
\hat{P}_{f}^{\mathrm{BH}}=\frac{1}{N_{s}} \sum_{k=1}^{N_{s}} \sum_{i=1}^{n} \mathbb{1}_{\left\{Y_{i}^{(k)}>T\right\}} \frac{\hat{g}\left(\boldsymbol{X}_{i}^{(k)}\right)}{\sum_{l=1}^{n} \hat{h}_{\mathrm{opt}}^{(l)}\left(\boldsymbol{X}_{i}^{(k)}\right)} \quad \text { with }\left\{\begin{array}{l}
\boldsymbol{X}_{i}^{(k)} \stackrel{\text { i.i.j. }}{\sim} \hat{h}_{\mathrm{opt}}^{(i)} \\
Y_{i}^{(k)}=\phi\left(\boldsymbol{X}_{i}^{(k)}\right)
\end{array} .\right.
$$




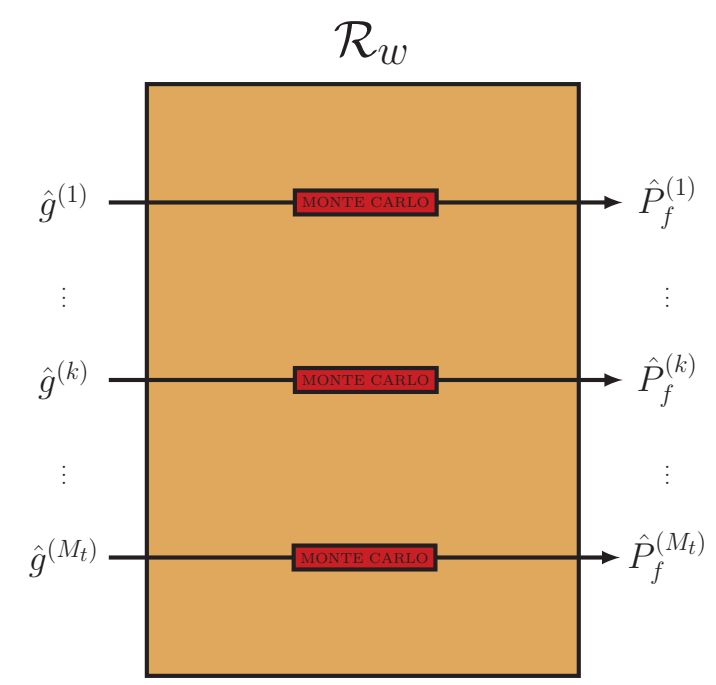

(a) ROSA with parallel CMC runs

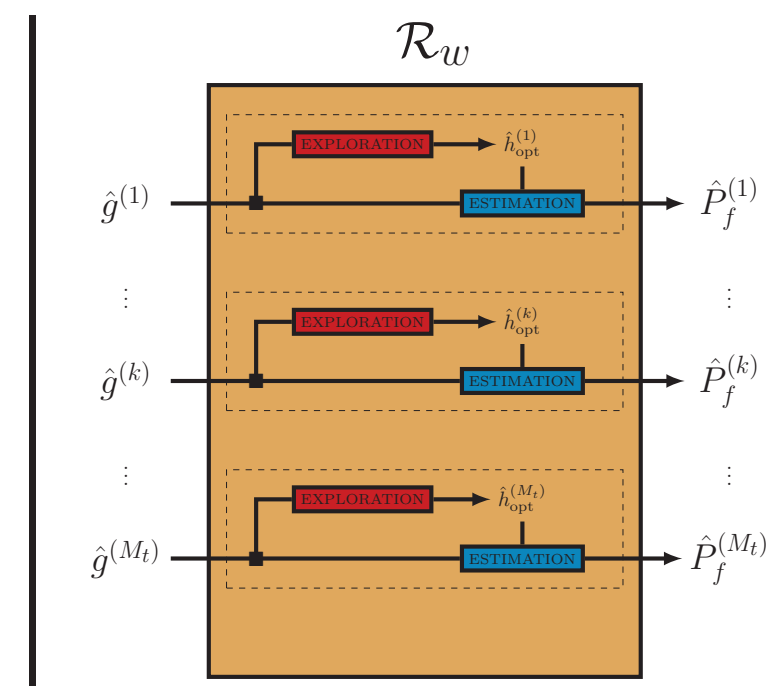

(b) ROSA with parallel IS runs

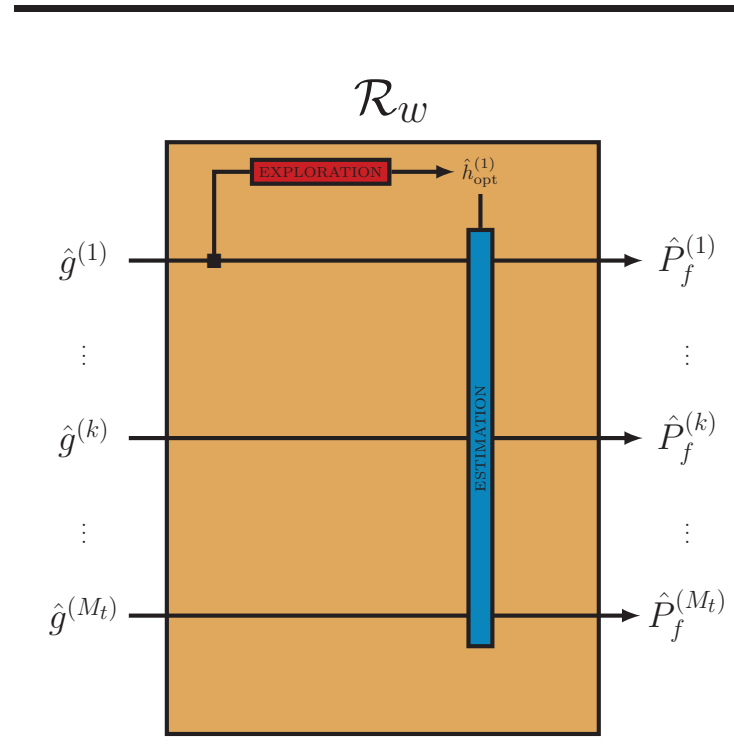

(c) ROSA with a single IS run

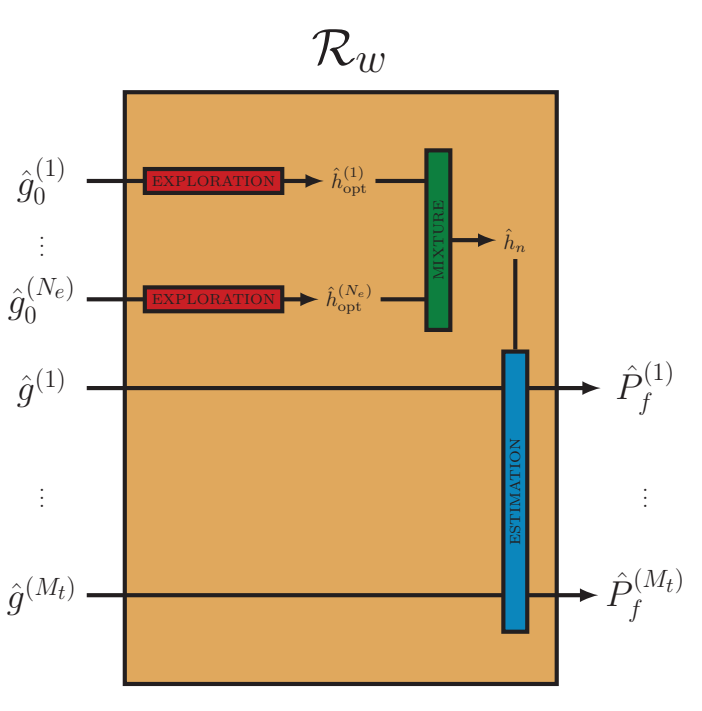

(d) ROSA with MIS after saving a few IS runs

Figure 8: Illustration of four computational strategies to perform ROSA 
In the particular case of the BH, MIS is equivalent to IS performed with the uniform mixture $\hat{h}_{n}$ of the auxiliary PDFs coming from the $n$ initial IS runs:

$$
\hat{P}_{f}^{\mathrm{BH}}=\frac{1}{N_{s}^{\prime}} \sum_{k=1}^{N_{s}^{\prime}} \mathbb{1}_{\left\{Y^{(k)}>T\right\}} \frac{\hat{g}\left(\boldsymbol{X}^{(k)}\right)}{\hat{h}_{n}\left(\boldsymbol{X}^{(k)}\right)} \quad \text { with }\left\{\begin{array}{l}
N_{s}^{\prime}=n \times N_{s} \\
\boldsymbol{X}^{(k)} \stackrel{\text { i.i.j. }}{\sim} \hat{h}_{n}=\frac{1}{n} \sum_{i=1}^{n} \hat{h}_{\mathrm{obs}}^{(i)} \\
Y^{(k)}=\phi\left(\boldsymbol{X}^{(k)}\right)
\end{array} .\right.
$$

To integrate MIS within the ROSA computational procedure already put in place, the following steps must be contemplated:

1. Repeat $\mathcal{B} \circ \mathcal{L}$ in order to get $n$ additional reassembled distributions $\hat{g}_{0}^{(i)}$.

2. Run IS for each $\hat{g}_{0}^{(i)}$ and retrieve both $\hat{h}_{\mathrm{opt}}^{(i)}$ and optimal samples $\left\{\begin{array}{l}\boldsymbol{X}_{i}^{(k)} \stackrel{i \text { i.i.d }}{\sim} \hat{h}_{\mathrm{opt}}^{(i)} \\ Y_{i}^{(k)}=\phi\left(\boldsymbol{X}^{(k)}\right)\end{array}\right.$.

3. Construct the mixture distribution $\hat{h}_{n}$ from the previous material.

4. Apply the MIS estimator $\hat{P}_{f}^{\mathrm{BH}}$ to all distributions $\hat{g}^{(i)}$ involved in the ROSA procedure.

To discern differences between the MIS method and the previous ones, the reader is invited to glance at Figure $8 \mathrm{~d}$ where a schematic illustration is proposed. Because $n$ IS explorations of $D_{f}$ are undertaken, the total number of calls to $\phi(\cdot)$ evolves accordingly and Eq. (72) turns into:

$$
N_{\text {tot }}=n \times N_{\text {iter }} \times N_{s}
$$

The complete algorithm used throughout the extensive simulation study that follows is synthesized in Figure 9. Of course, it contains the essential elements already presented in Figure 5 but more emphasis is given here to the computational strategy that must be implemented to be able to estimate thousands of failure probabilities. 


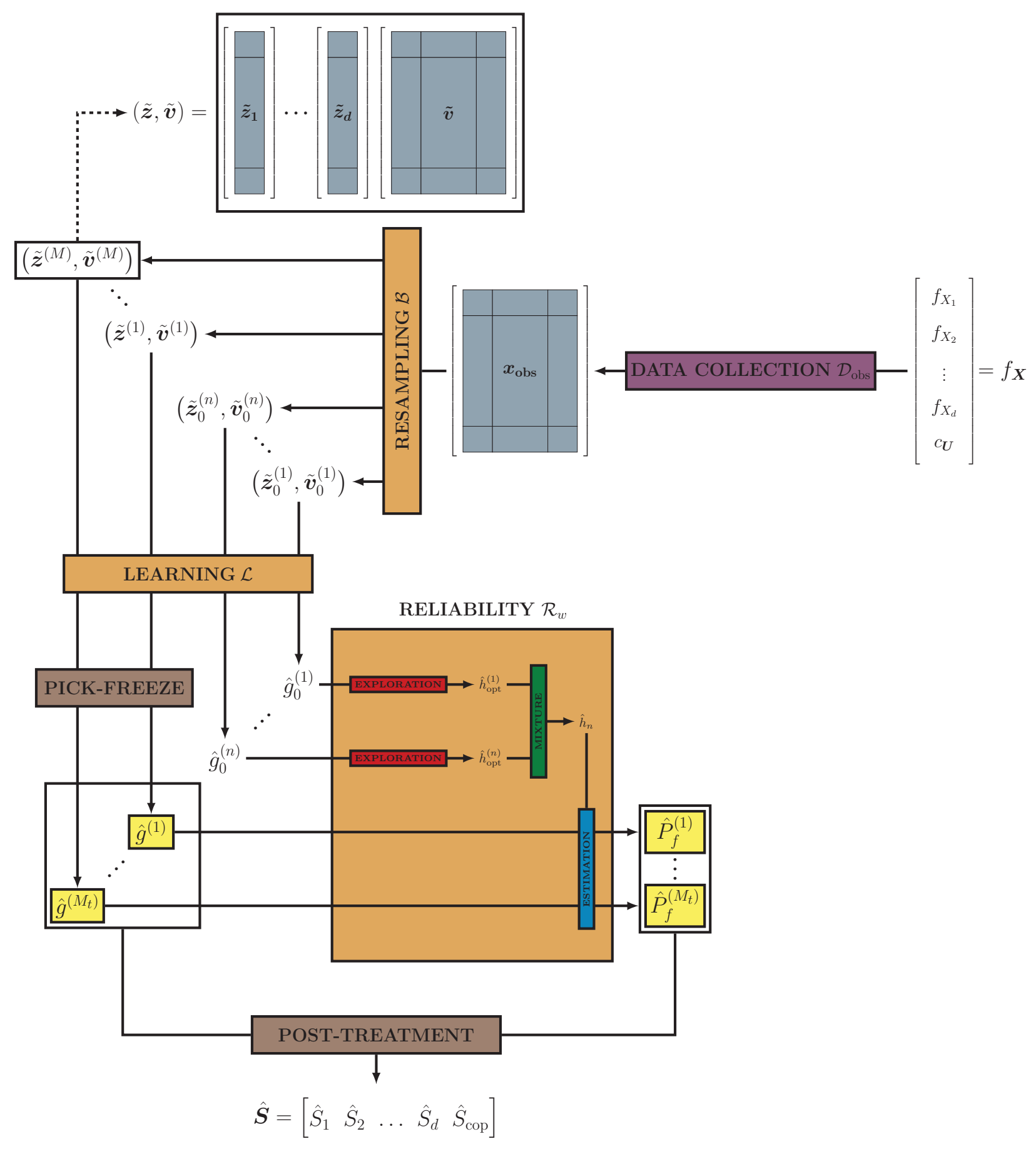

Figure 9: Illustration of all computational bricks when ROSA is performed with $\mathcal{R}_{w}$ 


\subsection{Application to a Gaussian nonlinear test case}

In this second test case, the theoretical failure probability $P_{f}$ is no longer computable with FORM. Using a stochastic reliability algorithms $\mathcal{R}_{w}$ such as IS is the only alternative to compute unbiased estimates. The main purpose here is to show that the computational strategy based on MIS provides the same results than the unaffordable sequential ROSA procedure which has the reliability algorithm $\mathcal{R}_{w}$ restarted for each new input PDF.

\subsubsection{Description}

The performance function $\phi(\cdot)$ includes now a nonlinear term:

$$
Y=\phi(\boldsymbol{X})=a_{1} X_{1}+a_{2} X_{2}+a_{3} X_{3}+a_{4} X_{1} X_{2},
$$

and the input vector $\boldsymbol{X}$ is again Gaussian with:

- Black box $\phi(\cdot)$ : $\quad a_{1}=a_{2}=2 ; a_{3}=a_{4}=1$

- Mean vector $\boldsymbol{m}$ : $\quad m_{1}=m_{2}=m_{3}=3$

- Covariance matrix $\boldsymbol{\Sigma}: \quad \sigma_{1}^{2}=\sigma_{2}^{2}=\sigma_{3}^{2}=1 ; \sigma_{12}=0.5 ; \sigma_{13}=\sigma_{23}=0.3$

When $T=50$, a brute-force CMC estimation of $P_{f}$ (with $10^{7}$ samples) leads to $\hat{P}_{f}^{\mathrm{CMC}}=7.8 \times 10^{-3}$. Since $\boldsymbol{X}$ is still Gaussian, switching into the standard normal space remains straightforward and the failure domain $D_{f}^{\mathcal{N}}$ may be written as follows:

$$
D_{f}^{\mathcal{N}}=\left\{\boldsymbol{w} \in \mathbb{R}^{d}: H(\boldsymbol{w})=\phi\left(\mathcal{T}^{-1}(\boldsymbol{w})\right)>T\right\} \quad \text { with } \quad \mathcal{T}^{-1}(\boldsymbol{w})=\boldsymbol{\Gamma} \boldsymbol{w}+\boldsymbol{m} \quad \text { and } \quad \boldsymbol{\Sigma}=\boldsymbol{\Gamma}^{\top}
$$

\subsubsection{Data-driven reliability assessment}

A Gaussian dataset $\boldsymbol{x}_{\mathrm{obs}}$ is simulated with $\mathcal{G}_{\boldsymbol{X}}$ but the underlying distribution $\mu_{\boldsymbol{X}}$ is pretended unknown thereafter. Because of this assumption, the learning model $\mathcal{M}$ is selected differently, just as a shrewd analyst would have done. Each marginal distribution $f_{X_{j}}$ is learnt with KDE and the associated bandwidth $\hat{h}_{j}$ is computed through Silverman's rule of thumb [142]. As far as copula estimation is concerned, it is decided to keep learning a Gaussian copula for reason of simplicity and also because it is expected to be sufficiently flexible when $d=3$. Thus, the estimated joint PDF is reassembled from the following components:

$$
\hat{f}_{X_{j}}\left(\cdot \mid \mathcal{M}_{j}, \tilde{\boldsymbol{z}}_{j}\right)=\hat{f}_{\mathrm{KDE}}\left(\cdot \mid \hat{h}_{j}, \tilde{\boldsymbol{z}}_{j}\right) \quad \text { and } \quad \hat{c}_{\boldsymbol{U}}\left(\cdot \mid \mathcal{M}_{\mathrm{cop}}, \tilde{\boldsymbol{v}}\right)=\hat{c}_{\mathcal{N}}(\cdot \mid \hat{\boldsymbol{\rho}}) \quad \text { with } \quad \hat{\boldsymbol{\rho}}=\hat{\boldsymbol{L}} \hat{\boldsymbol{L}}^{\top}
$$

Simplified notations are adopted with $k_{j}(\cdot)$ and $K_{j}(\cdot)$ replacing respectively $\hat{f}_{\mathrm{KDE}}\left(\cdot \mid \hat{h}_{j}, \tilde{\boldsymbol{z}}_{j}\right)$ and $\hat{F}_{\mathrm{KDE}}\left(\cdot \mid \hat{h}_{j}, \tilde{\boldsymbol{z}}_{j}\right)$. Note that for such a learning model $\mathcal{M}$, data uncertainty is no longer the only source of epistemic uncertainty. Indeed, estimating $f_{X_{1}}$ by KDE amounts to looking for the best representative of $f_{X_{1}}$ within a functional space that intersects the class of all Gaussian distributions without being 
embedded into it. Therefore, the Sobol index $S_{j}$ is supposed to measure both the uncertainty due to data scarcity and the uncertainty due to deciding to learn $f_{X_{1}}$ with KDE. Moreover, it is worth noting that model uncertainty is limited to the marginal distributions here. An example where it also affects the copula distribution is examined in Section 5. For a given joint PDF that has been learnt according to $\mathcal{M}$, the failure domain in the standard normal space is described by:

$$
\hat{D}_{f}^{\mathcal{N}}=\left\{\boldsymbol{w} \in \mathbb{R}^{d}: \hat{H}(\boldsymbol{w})=\phi\left(\hat{\mathcal{T}}^{-1}(\boldsymbol{w})\right)>T\right\}
$$

where the inverse Nataf transformation $\hat{\mathcal{T}}^{-1}=\psi_{1} \circ \psi_{2}$ has been adapted to the KDE framework in the following manner:

$$
\begin{aligned}
\hat{\mathcal{T}}^{-1}: & \mathbb{R}^{3} \stackrel{\mathbb{R}^{3}}{\longrightarrow} \quad \longrightarrow \mathbb{R}^{3} \\
& \boldsymbol{w} \stackrel{\psi_{1}}{\longrightarrow} \boldsymbol{v}=\hat{\boldsymbol{L}} \boldsymbol{w} \stackrel{\psi_{2}}{\longmapsto} \quad \boldsymbol{x} \quad \text { with } \quad x_{j}=K_{j}^{-1}\left(\Phi\left(v_{j}\right)\right)
\end{aligned}
$$

where $\psi_{1}$ introduces information about the estimated Gaussian copula and $\psi_{2}$ transforms Gaussian margins into KDE-like margins.

\subsubsection{More about the theoretical values of Sobol indices}

Just as in Section 3.5.3, the theoretical values of Sobol indices defined by Eq. (43) may be rewritten according to how the estimated joint PDF is specified:

$$
\begin{gathered}
\forall 1 \leq j \leq d, \quad S_{j}=\frac{\mathbb{V}\left(\mathbb{E}\left[P_{f}\left(\boldsymbol{Z}_{\mathrm{obs}}, \hat{\boldsymbol{h}}, \hat{\boldsymbol{\rho}}\right) \mid \hat{f}_{\mathrm{KDE}}\left(\cdot \mid \hat{h}_{j}, \boldsymbol{Z}_{\mathrm{obs}}^{[\bullet j]}\right)\right]\right)}{\mathbb{V}\left(P_{f}\left(\boldsymbol{Z}_{\mathrm{obs}}, \hat{\boldsymbol{h}}, \hat{\boldsymbol{\rho}}\right)\right)}, \\
S_{\mathrm{cop}}=\frac{\mathbb{V}\left(\mathbb{E}\left[P_{f}\left(\boldsymbol{Z}_{\mathrm{obs}}, \hat{\boldsymbol{h}}, \hat{\boldsymbol{\rho}}\right) \mid \hat{c}_{\mathcal{N}}(\cdot \mid \hat{\boldsymbol{\rho}})\right]\right)}{\mathbb{V}\left(P_{f}\left(\boldsymbol{Z}_{\mathrm{obs}}, \hat{\boldsymbol{h}}, \hat{\boldsymbol{\rho}}\right)\right)}
\end{gathered}
$$

where $\hat{\boldsymbol{h}}:=\left(\hat{h}_{1}, \hat{h}_{2}, \hat{h}_{3}\right)$. All four similarities mentioned when dealing with the Gaussian linear test case still hold here and hence it can be proved that $S_{1}=S_{2}$. The theoretical values are estimated with the "Pick-and-Freeze" scheme where all inputs training sets $\left\{\left(\boldsymbol{z}_{\text {obs }}^{(i)}, \boldsymbol{v}_{\text {obs }}^{(i)}\right)\right\}_{i=1}^{M}$ are simulated with the underlying generators $\mathcal{G}_{\boldsymbol{Z}}$ and $\mathcal{G}_{\boldsymbol{V}}$. The failure probabilities $\hat{P}_{f}^{(i)}$ are estimated sequentially with an IS algorithm as already illustrated in Figure 8b. This approach is used despite what has been previously said about its unworkable computational cost for realistic performance functions. There is a clear reason for this. Since the objective is to estimate the theoretical Sobol indices for comparative purposes, the methodology does not matter. Moreover, as $\phi(\cdot)$ is still analytical, the number of times it is evaluated is not of paramount importance. The chosen IS method consists in constructing a Gaussian auxiliary PDF in the vicinity of the MPFP. Such a technique is then referred to as Gaussian importance sampling (GIS). Further details may be found in Appendix B where it is fully explained why GIS works well. Each GIS run is based on $N_{s}=5 \times 10^{4}$ input particles so that each estimator 


\begin{tabular}{c|rcccc}
\hline \multicolumn{2}{c}{ ROSA with $\mathcal{G}_{\boldsymbol{Z}} / \mathcal{G}_{\boldsymbol{V}}:$} & $\hat{S}_{1}$ & $\hat{S}_{2}$ & $\hat{S}_{3}$ & $\hat{S}_{\text {cop }}$ \\
\hline \multirow{3}{*}{$N=300$} & mean : & $42.97 \%$ & $43.60 \%$ & $3.45 \%$ & $12.10 \%$ \\
& std : & $3.40 \%$ & $3.09 \%$ & $1.94 \%$ & $3.86 \%$ \\
& rank : & $(1)$ & $(1)$ & $(4)$ & $(3)$ \\
\hline
\end{tabular}

Table 4: Estimation of Sobol indices by means of the unknown generators $\mathcal{G}_{\boldsymbol{Z}}$ and $\mathcal{G}_{\boldsymbol{V}}$

$\hat{P}_{f}^{(i)}$ might have the smaller possible variance. With such a computational effort, ROSA performed with sequential GIS runs is intended to be as accurate as ROSA performed with sequential runs of a deterministic reliability algorithm $\mathcal{R}$ that would be able to compute all exact value $P_{f}^{(i)}$. For a sample size $N=300$, Sobol indices are estimated $N_{\text {rep }}=50$ times and the results are shown in Table 4 . As expected, one can notice that $\hat{S}_{1} \approx \hat{S}_{2}$. The rankings are very similar to those obtained in Table 1 for the Gaussian linear test case. However, the preeminence of both $\hat{S}_{1}$ and $\hat{S}_{2}$ has been strengthened while $\hat{S}_{\text {cop }}$ no longer belongs to the leading group of indices.

\subsubsection{Impact of the additional uncertainty induced by $\mathcal{R}_{w}$}

The aim of this subsection is to ensure that the forecast uncertainty introduced by $\mathcal{R}_{w}$ does not deteriorate too much the estimation of Sobol indices. Let us go back to the situation faced in practice where the only provided material is $\boldsymbol{x}_{\mathrm{obs}}$. All ROSA procedures are conducted with the "inversereverse" trick discussed in Section 4.2 and illustrated on Figure 8c. GIS is run once and the associated auxiliary PDF $\boldsymbol{x} \longmapsto \varphi_{3}\left(\boldsymbol{x}-\hat{\boldsymbol{x}}_{1}^{\star}\right)$ is used to estimated all other failures probabilities:

$$
\hat{P}_{f}^{(i)}=\frac{1}{N_{s}} \sum_{k=1}^{N_{s}} \mathbb{1}_{\left\{Y^{(k)}>T\right\}}\left(\boldsymbol{X}^{(k)}\right) \frac{\hat{g}^{(i)}\left(\boldsymbol{X}^{(k)}\right)}{\varphi_{3}\left(\boldsymbol{X}^{(k)}-\hat{\boldsymbol{x}}_{1}^{\star}\right)} \quad \text { with }\left\{\begin{array}{l}
\boldsymbol{X}^{(k)} \stackrel{\text { i.i.i.d }}{\sim} \mathcal{N}\left(\hat{\boldsymbol{x}}_{1}^{\star}, \boldsymbol{I}_{3}\right) \\
Y^{(k)}=\phi\left(\boldsymbol{X}^{(k)}\right)
\end{array}\right.
$$

It is straightforward to see that:

$$
\begin{aligned}
& \mathbb{E}\left[\hat{P}_{f}^{(i)}\right]=P_{f}^{(i)}=\int_{D_{f}} \hat{g}^{(i)}(\boldsymbol{x}) \mathrm{d} \boldsymbol{x}, \\
& \mathbb{V}\left(\hat{P}_{f}^{(i)}\right)=\frac{V_{i}}{N_{s}}=\frac{1}{N_{s}} \mathbb{V}\left(\mathbb{1}_{D_{f}}(\boldsymbol{X}) \frac{\hat{g}^{(i)}(\boldsymbol{X})}{\varphi_{3}\left(\boldsymbol{X}-\hat{\boldsymbol{x}}_{1}^{\star}\right)}\right) \quad \text { with } \quad \boldsymbol{X} \sim \mathcal{N}\left(\hat{\boldsymbol{x}}_{1}^{\star}, \boldsymbol{I}_{3}\right) .
\end{aligned}
$$

$\mathbb{V}\left(\hat{P}_{f}^{(i)}\right)$ mainly depends on how large $N_{s}$ is. In Section 4.4.3, GIS is considered as a pseudo-deterministic reliability algorithm because the use of $N_{s}=5 \times 10^{4}$ particles allows to eliminate almost all forecast uncertainty. On the contrary, if $N_{s}$ is lowered, the additional uncertainty due to the particles $\left\{\boldsymbol{X}^{(k)}\right\}_{k=1}^{N_{s}}$ rises. If so, the random variables $\hat{P}_{f}(\tilde{\boldsymbol{Z}}, \tilde{\boldsymbol{V}})$ and $P_{f}(\tilde{\boldsymbol{Z}}, \tilde{\boldsymbol{V}})$ may have very different distributions. In practice, over the ROSA procedure, there is a risk that the sequence $\left\{\hat{P}_{f}^{(i)}\right\}_{i=1}^{M_{t}}$ that gathers all estimated failure probabilities and the sequence $\left\{P_{f}^{(i)}\right\}_{i=1}^{M_{t}}$ that gathers all exact failure probabilities 
lead to dissimilar conclusions. The ROSA algorithm is run $N_{\text {rep }}=10$ times with $N_{s}$ taking a decreasing series of values. The results are shown in Table 5 . When $N_{s}=5 \times 10^{3}$, the estimated Sobol indices are very close to those obtained in Table 4 after conducting a ROSA procedure fueled by the underlying generators $\mathcal{G}_{\boldsymbol{Z}}$ and $\mathcal{G}_{\boldsymbol{V}}$ and relying on sequential runs of the GIS algorithm. Three lessons may be drawn from this:

1. The data-driven ROSA algorithm fulfills its initial objective because accurate estimates of the theoretical indices are derived from the only knowledge of $\boldsymbol{x}_{\mathrm{obs}}$. It is positive to note that using the resampling mechanism $\mathcal{B}$ does not introduce any blatant bias.

2. Using a sampling-based reliability algorithm $\mathcal{R}_{w}$ does not disturb ROSA provided that the additional uncertainty introduced by rare-event simulations remains small compared with the initial learning uncertainty. For $N_{s}=5 \times 10^{3}$, it can be easily verified that:

$$
\mathbb{V}\left(\hat{P}_{f}^{(i)}\right)=\frac{V_{i}}{N_{s}} \ll \mathbb{V}\left(\hat{P}_{f}(\tilde{\boldsymbol{Z}}, \tilde{\boldsymbol{V}})\right) \text { where }(\tilde{\boldsymbol{Z}}, \tilde{\boldsymbol{V}}) \sim\left(\hat{\mu}_{\boldsymbol{Z} \boldsymbol{V}}\left[\boldsymbol{x}_{\mathrm{obs}}\right]\right)^{\otimes N}
$$

3. The computational strategy described by Eq. (86) is effective since it allows to perform ROSA accurately with an alleviated simulation budget. With the "inverse-reverse" trick, the overall computational cost only amounts to $N_{\text {tot }}=N^{\star}+N_{s}$ calls to $\phi(\cdot)$ where $N^{\star}$ is the number of calls consumed by FORM to find $\hat{\boldsymbol{x}}_{1}^{\star}$.

When $N_{s}$ becomes smaller (for instance $100 \leq N_{s} \leq 10^{3}$ ), the mean values of Sobol indices are still almost unbiased. It emphasizes the fact that GIS is an appropriate method to address reliability analysis even when it only incorporates a limited number of particles. In addition, it reinforces the idea that using one single auxiliary PDF is quite enough. However, it must be observed that the dispersion of the estimated Sobol indices tends to soar as $N_{s}$ decreases. When less than $N_{s}=500$ particles are used, the standard deviations exceed $10 \%$ for large indices. When $N_{s}$ becomes even smaller, the estimators suffer further deterioration. In such a situation, no one would venture trusting the ROSA results and drawing definitive conclusions from them. A key idea is thus to understand that ROSA can be performed with a sampling-based reliability method $\mathcal{R}_{w}$ provided that it is unbiased and sufficiently robust.

Remark. In Table 5, all mean values and standard deviations are obtained after running $N_{\text {rep }}$ times the ROSA algorithm on the dataset under study. Unlike what was said in the concluding remark of Section 3.5.4, it is here necessary to do so and it cannot be avoided. Dispersion is properly assessed only if it takes into account the uncertainty due to bootstrap resampling and the uncertainty due to the change of auxiliary PDF. If new sequences of training sets $\left\{\left(\tilde{\boldsymbol{z}}^{\left(i_{k}\right)}, \tilde{\boldsymbol{v}}^{\left(i_{k}\right)}\right)\right\}_{k=1}^{M}$ are derived from the first sequence $\left\{\left(\tilde{\boldsymbol{z}}^{(i)}, \tilde{\boldsymbol{v}}^{(i)}\right)\right\}_{i=1}^{M}$, the first uncertainty is propagated but the second one is not. Therefore, 


\begin{tabular}{|c|c|c|c|c|c|c|}
\hline \multicolumn{3}{|c|}{ ROSA with $\mathcal{B}$ : } & $\hat{S}_{1}$ & $\hat{S}_{2}$ & $\hat{S}_{3}$ & $\hat{S}_{\mathrm{cop}}$ \\
\hline \multirow{18}{*}{ GIS } & \multirow{3}{*}{$N_{s}=5 \times 10^{3}$} & mean : & $41.83 \%$ & $41.40 \%$ & $2.50 \%$ & $17.01 \%$ \\
\hline & & std : & $3.20 \%$ & $3.02 \%$ & $1.40 \%$ & $3.66 \%$ \\
\hline & & rank : & (1) & (1) & (4) & (3) \\
\hline & \multirow{3}{*}{$N_{s}=10^{3}$} & mean : & $41.96 \%$ & $39.54 \%$ & $1.96 \%$ & $16.57 \%$ \\
\hline & & std : & $6.90 \%$ & $5.68 \%$ & $1.57 \%$ & $3.20 \%$ \\
\hline & & rank: & (1) & $(2)$ & (4) & (3) \\
\hline & \multirow{3}{*}{$N_{s}=500$} & mean : & $40.78 \%$ & $41.00 \%$ & $3.94 \%$ & $15.91 \%$ \\
\hline & & std : & $9.28 \%$ & $8.45 \%$ & $2.29 \%$ & $3.25 \%$ \\
\hline & & rank : & $(2)$ & $(1)$ & (4) & (3) \\
\hline & \multirow{3}{*}{$N_{s}=100$} & mean : & $39.29 \%$ & $44.64 \%$ & $2.80 \%$ & $12.39 \%$ \\
\hline & & std : & $18.26 \%$ & $19.23 \%$ & $1.62 \%$ & $3.89 \%$ \\
\hline & & rank: & $(2)$ & $(1)$ & $(4)$ & (3) \\
\hline & \multirow{3}{*}{$N_{s}=50$} & mean : & $38.54 \%$ & $38.15 \%$ & $4.60 \%$ & $18.11 \%$ \\
\hline & & std : & $12.14 \%$ & $12.82 \%$ & $2.15 \%$ & $5.60 \%$ \\
\hline & & rank : & $(1)$ & $(2)$ & $(4)$ & (3) \\
\hline & \multirow{3}{*}{$N_{s}=10$} & mean : & $47.16 \%$ & $36.55 \%$ & $7.57 \%$ & $14.20 \%$ \\
\hline & & std : & $33.29 \%$ & $30.97 \%$ & $8.34 \%$ & $11.37 \%$ \\
\hline & & rank : & (1) & $(2)$ & (4) & (3) \\
\hline
\end{tabular}

Table 5: Impact on the ROSA results of a gradual decline in GIS robustness 
to succeed in propagating simultaneously both uncertainties, the only solution is to repeat the overall ROSA procedure several times.

\subsubsection{Comparison of several ROSA algorithms}

The idea is now to verify whether the MIS approach is a trustworthy option in order to perform ROSA. The simulation study is based on the dataset $\boldsymbol{x}_{\mathrm{obs}}$ that has already been used to produce the results found in Table 5. An additional IS solution is introduced in this subsection. Indeed, a more flexible way of constructing an auxiliary PDF might be to use the nonparametric adaptive importance sampling (NAIS) algorithm designed in [137]. In this test case, for any input PDF $\hat{g}^{(i)}$, NAIS needs $N_{\text {iter }}=3$ iterations to construct a weighted KDE estimate $\hat{h}_{\mathrm{opt}}^{(i)}$ of the optimal auxiliary PDF $h_{\mathrm{opt}}^{(i)}$. For comparative purposes, ROSA is performed in three different manners:

(St1) Sequential runs of FORM

$$
\begin{array}{llll}
(\mathrm{St} 1) & \text { Sequential runs of FORM } & \Rightarrow & N_{\text {tot }}=(d+2) \times M \times N^{\star}=500 \times 10^{3} \\
\text { (St2) } & \text { MIS based on } n=5 \text { GIS runs } & \Rightarrow & N_{\text {tot }}=n \times N_{\text {IS }}=n \times\left(N^{\star}+N_{s}\right) \approx 25 \times 10^{3} ;
\end{array} ;
$$$$
\text { (St3) MIS based on } n=5 \text { NAIS runs } \Rightarrow \quad N_{\text {tot }}=n \times N_{\text {IS }}=n \times\left(N_{\text {iter }} \times N_{s}\right)=75 \times 10^{3} \text {. }
$$

The computational costs are calculated with $d=3, M=10^{3}, N^{\star}=10^{2}, N_{s}=10^{3}$ and $N_{\text {iter }}=3$. Each scenario is repeated $N_{\text {rep }}=10$ times and the results are presented in Table 6 . It can be seen that the choice of the reliability algorithm has little influence on the estimation of Sobol indices. The results obtained with (St2) and (St3) are very close to the theoretical values. This shows that combining a MIS strategy with either GIS or NAIS fulfills the declared objective. Even if the inner workings of these two IS techniques are different, both are well adapted to the Gaussian nonlinear test case. NAIS is maybe too sophisticated here and GIS seems to be a much easier way of performing reliability assessment. The fact that both IS techniques behave in a similar way is positive because this highlights the fact that any IS approach may be implemented within the ROSA procedure as long as it is sufficiently robust. One can also notice that (St1) provides accurate estimates of Sobol indices. It is rather surprising because it has been verified numerically that the FORM algorithm results in a slight overestimation of $P_{f}$. In this nonlinear test case, the forecast error due to the linear approximation of the limit state in the standard normal space is not large enough to impact the distribution of $\hat{P}_{f}(\tilde{\boldsymbol{Z}}, \tilde{\boldsymbol{V}})$. This explains why using FORM unexpectedly delivers the right results. Because of this, it is decided to apply the ROSA algorithm to a physical test case where no information is provided about the input probabilistic model and where $\phi(\cdot)$ is much more complicated. 


\begin{tabular}{|c|c|c|c|c|c|c|c|c|c|}
\hline \multicolumn{6}{|c|}{ ROSA with $\mathcal{B}$ : } & $\hat{S}_{1}$ & $\hat{S}_{2}$ & $\hat{S}_{3}$ & $\hat{S}_{\text {cop }}$ \\
\hline \multirow{3}{*}{ 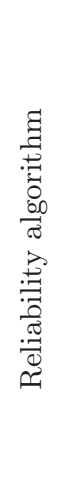 } & $\mathcal{R}$ & FORM & & & $\begin{array}{r}\text { mean : } \\
\text { std : } \\
\text { rank : }\end{array}$ & $\begin{array}{c}42.19 \% \\
3.99 \% \\
(2)\end{array}$ & $\begin{array}{c}43.68 \% \\
3.70 \% \\
(1)\end{array}$ & $\begin{array}{c}3.18 \% \\
1.53 \% \\
(4)\end{array}$ & $\begin{array}{c}10.64 \% \\
3.92 \% \\
(3)\end{array}$ \\
\hline & \multirow{2}{*}{$\mathcal{R}_{w}$} & GIS & \multirow{2}{*}{ 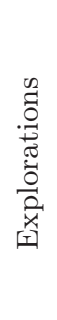 } & $n=5$ & $\begin{array}{r}\text { mean : } \\
\text { std : } \\
\text { rank : }\end{array}$ & $\begin{array}{c}41.75 \% \\
2.63 \% \\
(2)\end{array}$ & $\begin{array}{c}42.55 \% \\
3.53 \% \\
(1)\end{array}$ & $\begin{array}{c}3.67 \% \\
2.82 \% \\
(4)\end{array}$ & $\begin{array}{c}17.29 \% \\
3.32 \% \\
(3)\end{array}$ \\
\hline & & NAIS & & $n=5$ & $\begin{array}{r}\text { mean : } \\
\text { std : } \\
\text { rank : }\end{array}$ & $\begin{array}{c}40.75 \% \\
2.99 \% \\
(2)\end{array}$ & $\begin{array}{c}43.93 \% \\
3.83 \% \\
(1)\end{array}$ & $\begin{array}{c}3.23 \% \\
3.08 \% \\
(4)\end{array}$ & $\begin{array}{c}17.95 \% \\
3.15 \% \\
(3)\end{array}$ \\
\hline
\end{tabular}

Table 6: Estimation of Sobol indices with the ROSA procedure by means of three reliability algorithms

\section{Application to the buckling of a composite plate}

\subsection{Description}

After experimenting the proposed methodology on two purely analytical test cases, let us now consider a real-life simulation code $\phi(\cdot)$ that provides information about the elastic behavior of a laminate composite plate. Plated structures are major components in most industrial facilities and have been widely studied in the literature to meet the needs of aerospace, civil and offshore engineering. Depending on its dimensions, the plate may be poorly or higly sensitive to compressive loads. The sudden loss of stability due to external compressive efforts is called buckling and may cause disastrous outcomes in terms of structural safety [143]. Let us imagine a square plate $(a / b=1)$ simply supported on its four edges. The vertical translation is blocked but all other five degrees of freedom remain possible. A linear compressive stress $L_{x}$ is applied on both sides of the plate and along the $x$-axis. An illustration may be found on the left part of Figure 10. It is a rather classical configuration, for example to study the buckling phenomenon occurring between the stiffeners of aircraft wing panels. In this aeronautical context, the plate is seldom square but the assumption is kept here in order to alleviate formulas. The plate is made of a 8-ply carbon/epoxy composite laminate. It consists of an assembly of fibrous composite materials that are bonded together in order to acquire enhanced physical properties, in particular outstanding stiffness-to-density ratios. The laminate plate presents mirror symmetry, which means that its midplane is a symmetry plane of the layer stacking. In that respect, two layers that are positioned at an equal distance of the midplane have their fibers oriented in the same direction $\theta$ (see the last row in Table 7).

In the rest of this case study, the retained modeling implies two major assumptions: (a) there is no coupling between the membrane behavior and the bending behavior, and (b) the bending behavior 


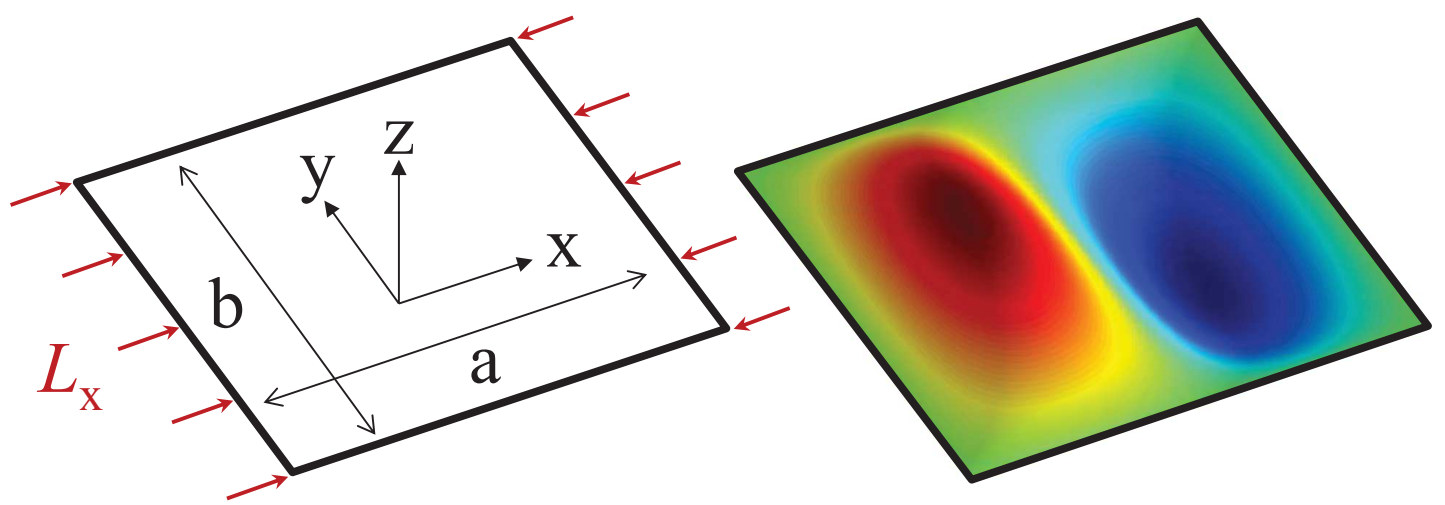

Figure 10: Buckling of a simply supported plate under uniaxial loading. The buckling mode is $\left(p^{\star}, q^{\star}\right)=(2,1)$. The colormap corresponds to the displacement in the $z$-direction.

is orthotropic [144]. In order to determine resultant forces and moments, one has to construct the following stiffness matrix:

$$
\left[\begin{array}{c|c}
\boldsymbol{A} & \boldsymbol{B} \\
\hline \boldsymbol{B} & \boldsymbol{D}
\end{array}\right] \in \mathbb{R}^{6 \times 6}
$$

where $\boldsymbol{A} \in \mathbb{R}^{3 \times 3}$ is the membrane stiffness matrix, $\boldsymbol{B} \in \mathbb{R}^{3 \times 3}$ is the membrane/bending coupling matrix and $\boldsymbol{D} \in \mathbb{R}^{3 \times 3}$ is the bending stiffness matrix. For further details, the reader is invited to consult [145]. One has $\boldsymbol{B}=\mathbf{0}$ because of what is assumed in (a) while at the same time the orthotropic behavior highlighted in (b) allows to reduce to zero several coefficients in $\boldsymbol{D}$. In addition, as $\boldsymbol{D}$ is a symmetric positive definite matrix, its final expression only involves four relevant coefficients:

$$
\boldsymbol{D}=\left[\begin{array}{ccc}
D_{11} & D_{12} & D_{13} \\
D_{21} & D_{22} & D_{23} \\
D_{31} & D_{32} & D_{33}
\end{array}\right]=\left[\begin{array}{ccc}
D_{11} & D_{12} & 0 \\
D_{12} & D_{22} & 0 \\
0 & 0 & D_{33}
\end{array}\right]=\left[\begin{array}{ccc}
X_{1} & X_{4} & 0 \\
X_{4} & X_{2} & 0 \\
0 & 0 & X_{3}
\end{array}\right]
$$

The focus is then exclusively on $\boldsymbol{D}$ since its coefficients suffice to predict whether or not buckling will take place. A buckling mode is a wave-shaped deformation of the plate which is characterized by $p$ half-waves in the $x$-direction and $q$ half-waves in the $y$-direction. An illustration for the particular case where $p=2$ and $q=1$ is presented on the right part of Figure 10. For a given mode $(p, q) \in\left(\mathbb{N}^{\star}\right)^{2}$, the buckling load is estimated using the following closed-form solution :

$$
N_{0}(p, q)=\frac{\pi^{2}}{p^{2} a^{2}}\left[p^{4} D_{11}+2 p^{2} q^{2}\left(D_{12}+2 D_{3}\right)+q^{4} D_{22}\right] .
$$

The buckling factor $\Lambda_{p, q}$ associated to a given buckling mode $(p, q)$ is the ratio between its critical load and the actual applied load:

$$
\Lambda_{p, q}=\frac{N_{0}(p, q)}{L_{x}}
$$


It may be seen as a factor of safety that indicates how many times $L_{x}$ has to be multiplied to result in the specified buckling mode. In case buckling happens, the plate adopts the mode $\left(p^{\star}, q^{\star}\right)$ that corresponds to the minimum buckling factor over all possible modes:

$$
\Lambda:=\Lambda_{p^{\star}, q^{\star}}=\min _{p, q} \Lambda_{p, q}=\phi(\boldsymbol{D}) .
$$

For a given loading $L_{x}$, two typical situations are then encountered:

$$
\left\{\begin{array}{l}
\Lambda>1 \Rightarrow \text { Buckling is not predicted. } \\
\Lambda \leq 1 \Rightarrow \text { Buckling occurs. }
\end{array}\right.
$$

From Eq. (93) and Eq. (94), it is straightforward to see that an increase in $L_{x}$ lowers $\mathbb{E}[\Lambda]$ and raises the failure probability $\mathbb{P}\{\Lambda \leq 1\}$. In this test case, as it is highlighted in the right-hand side of Eq. (94), the performance function $\phi$ takes $\boldsymbol{D}$ as input and yields $\Lambda$ as output. The relevancy of this test case is mainly due to the fact that there is a lack of knowledge about the input probability measure $\mu_{\boldsymbol{D}}$. However, basic notions in mechanics of composite materials allow to express the bending stiffness matrix $\boldsymbol{D}$ in terms of better-known physical parameters. Thus, one has:

$$
\boldsymbol{D}=\phi_{0}\left(E_{11}, E_{22}, \nu_{12}, G_{12}, e, \Theta_{1}, \ldots, \Theta_{8}\right)
$$

with $\phi_{0}(\cdot)$ another deterministic simulation code. All variables involved in the above equation are assumed independent and they are specified in Table 7. One can therefore understand that aleatory uncertainty on $\boldsymbol{D}$ is attributable to the combined effect of the dispersion observed on the elastic properties of the ply material and the existence of small random errors in the ply orientations. At this step, a notable assumption is to consider that all plies have exactly the same elastic behavior, hence the same Young's modulus and so on. Moreover, it is also assumed that the bending behavior remains orthotropic in presence of those uncertainties, and the simplification brought in Eq. (91) still holds.

\begin{tabular}{c|l|c|cc}
\hline Input & Description (Unit) & Type & \multicolumn{2}{|c}{ Parameters } \\
\hline$E_{11}$ & Young's modulus in fiber direction (MPa) & $\mathcal{N}\left(m_{1}, \sigma_{1}^{2}\right)$ & $m_{1}=1.81 \times 10^{5}$ & $\frac{\sigma_{1}}{m_{1}}=0.01$ \\
$E_{22}$ & Young's modulus in transverse direction (MPa) & $\mathcal{N}\left(m_{2}, \sigma_{2}^{2}\right)$ & $m_{2}=1.03 \times 10^{4}$ & $\frac{\sigma_{2}}{m_{2}}=0.02$ \\
$G_{12}$ & Shear modulus (MPa) & $\mathcal{N}\left(m_{3}, \sigma_{3}^{2}\right)$ & $m_{3}=7.17 \times 10^{3}$ & $\frac{\sigma_{3}}{m_{3}}=0.04$ \\
$\nu_{12}$ & Poisson's ratio (1) & $\mathcal{N}\left(m_{4}, \sigma_{4}^{2}\right)$ & $m_{4}=0.28$ & $\frac{\sigma_{4}}{m_{4}}=0.01$ \\
\hline$e$ & Thickness (mm) & $\mathcal{N}\left(m_{e}, \sigma_{e}^{2}\right)$ & $m_{e}=1.52$ & $\frac{\sigma_{e}}{m_{e}}=0.002$ \\
\hline$\Theta_{i}$ & Fibers orientations $\left(^{\circ}\right)$ & $\mathcal{U}\left(\left[\theta_{i} \pm 2^{\circ}\right]\right)$ & $\theta_{i} \in\{45,0,-45,90,90,-45,0,45\}$ \\
\hline
\end{tabular}

Table 7: Probabilistic models chosen to represent aleatory uncertainties on physical variables

In this situation, the joint PDF $f_{\boldsymbol{D}}$ can not be derived analytically but $\boldsymbol{D}$ can be simulated if the state of knowledge provided in Table 7 is combined with Eq. (95). It is therefore possible to estimate 


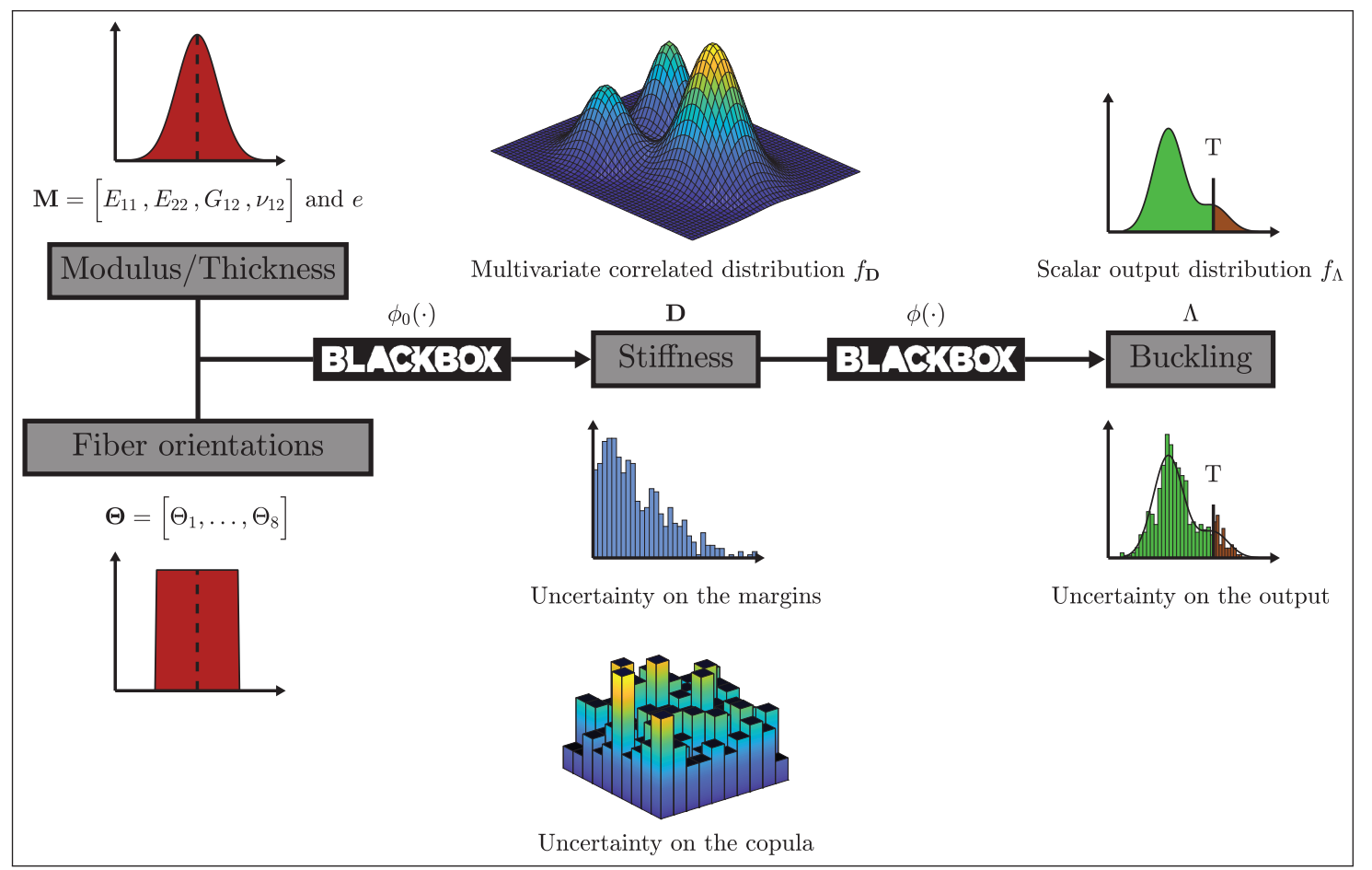

Figure 11: Overview of uncertainty modeling for the buckling of a composite laminate plate.

$P_{f}=\mathbb{P}\{\Lambda<1\}$ with the two-step sampling approach presented in Figure 11. A brute-force CMC estimation thus gives $\hat{P}_{f}^{\mathrm{CMC}}=4.6 \times 10^{-3}$ when one has $a=10^{3} \mathrm{~mm}$ and $L_{x}=485 \mathrm{~N} / \mathrm{mm}$. The change of notation suggested in Eq. (91) and amounting to replace $\boldsymbol{D} \in \mathbb{R}^{9}$ by $\boldsymbol{X} \in \mathbb{R}^{4}$ becomes effective in what follows in order to stick to the formalism used from the very start of this paper.

\subsection{Construction of a probabilistic model for the joint distribution of bending stiffness coefficients}

Within the established framework, the lack of knowledge about the underlying type of distribution concerns here both the margins and the copula. KDE is again used to learn all marginal distributions. Unlike what was done in Sections 3.5 and 4.4 where a Gaussian copula is estimated with MLE, copula estimation is here conducted differently since there is no reason to believe that the whole dependence structure in $\mathcal{H}_{4}$ is well described by such a light-tail copula family. To enable an accurate and flexible assessment of the distribution tails, it is preferred to switch to regular vine copulas (R-vines). They are one popular solution (among many others) to tackle copula estimation in higher dimension [146]. Many recent works insist on their usefulness to grasp most intricacies existing within a multivariate dependence pattern $[75,147,148]$. Their tree-based structure responds to the lack of polymorphism observed on both elliptical and Archimedean copulas. Using R-vines is of great interest in this study because learning uncertainty is likely to affect at the same time: (a) the tree structure $\hat{\boldsymbol{T}}$, (b) the 
pair-copula families $\hat{\boldsymbol{F}}$ and (c) their associated parameters $\hat{\boldsymbol{\theta}}$. Over the ROSA process, several R-vine models are thus expected to be observed under the effects of learning uncertainty. To recapitulate, the joint PDF of bending stiffness coefficients can be estimated with the following learning model:

$$
\hat{f}_{X_{j}}\left(\cdot \mid \mathcal{M}_{j}, \tilde{z}_{j}\right)=\hat{f}_{\mathrm{KDE}}\left(\cdot \mid \hat{h}_{j}, \tilde{z}_{j}\right) \quad \text { and } \quad \hat{c}_{\boldsymbol{U}}\left(\cdot \mid \mathcal{M}_{\mathrm{cop}}, \tilde{\boldsymbol{v}}\right)=\hat{c}_{\mathrm{RV}}(\cdot \mid \hat{\boldsymbol{T}}, \hat{\boldsymbol{F}}, \hat{\boldsymbol{\theta}})
$$

\subsection{Results}

A small-sized dataset $\boldsymbol{x}_{\mathrm{obs}} \in \mathbb{R}^{N \times d}$ is set up. It is compound of $N=300$ simulated bending stiffness matrices which are reduced to their $d=4$ relevant coefficients. The ROSA algorithm based on MIS is applied $N_{\text {rep }}=10$ times and the averaged values are presented in Table 8. The settings validated beforehand are kept unchanged for this test case. In particular, $M=10^{3}$ bootstrap replications are simulated to fuel the "Pick-and-Freeze" estimation scheme $\mathcal{A}$. Regarding $\mathcal{R}_{w}, n=5$ NAIS runs lead to an auxiliary PDF $\hat{h}_{n}$ with the MIS method. As each NAIS run is based on $N_{s}=5 \times 10^{3}$ input particles and requires $N_{\text {iter }}=3$ iterations to be able to construct $\hat{h}_{\mathrm{opt}}^{(i)}$, the total number of calls to $\phi(\cdot)$ is given by Eq. (79) and is equal to:

$$
N_{\text {tot }}=n \times N_{\text {iter }} \times N_{s}=5 \times 3 \times\left(5 \times 10^{3}\right)=75 \times 10^{3} .
$$

The copula distribution $\hat{c}_{U}$ is the most influential component since it is responsible for more than a half of the variance observed on the reliability estimator $\hat{P}_{f}(\tilde{\boldsymbol{Z}}, \tilde{\boldsymbol{V}})$. The next two most influential components are $\hat{f}_{X_{4}}$ and $\hat{f}_{X_{1}}$. On the contrary, $\hat{f}_{X_{2}}$ and $\hat{f}_{X_{3}}$ have much smaller indices. Moreover, the standard deviations of all Sobol indices remain at the same low level than in the previous cases. This provides further support for the claim that the sensitivity indices computed with the ROSA algorithm only quantify the impact of the epistemic uncertainties conveyed by statistical learning. Those indices are not subject to the undesirable uncertainty coming from how well $\hat{h}_{n}$ is trained. The results collected in Table 8 give further proof of the attention that must be paid to copula estimation in reliability analysis. In this particular case where the copula distribution is the main contributor for uncertainty in reliability assessment, targeted data assimilation no longer makes sense. Indeed, if one is willing to collect additional data for the copula, a prerequisite is always to collect additional joint observations in $\mathbb{R}^{d}$. If such an operation is undertaken, additional observations are then also available to improve the state of knowledge about all marginal distributions. In that way, if the copula is identified as the leading entity in the propagation of epistemic uncertainties, the practitioner has to face a dilemma. On the one hand, a practical expedient may consist in collecting observations for the next most influential margin. However, there are strong concerns that variance reduction will be barely visible. On the other hand, one has to prospect for other joint observations in order to create a larger dataset, which is often a time-wasting process. 


\begin{tabular}{|c|c|c|c|c|c|c|}
\hline \multicolumn{2}{|c|}{ ROSA with $\mathcal{B}$} & $\hat{S}_{1}$ & $\hat{S}_{2}$ & $\hat{S}_{3}$ & $\hat{S}_{4}$ & $\hat{S}_{\text {cop }}$ \\
\hline $\boldsymbol{x}_{\mathrm{obs}}$ & mean & $20.95 \%$ & $15.08 \%$ & $7.84 \%$ & $23.65 \%$ & $38.51 \%$ \\
\hline$N=300$ & $\begin{array}{r}\text { std } \\
\text { rank }\end{array}$ & $\begin{array}{c}1.54 \% \\
(3)\end{array}$ & $\begin{array}{c}1.57 \% \\
(4)\end{array}$ & $\begin{array}{c}1.02 \% \\
(5)\end{array}$ & $\begin{array}{c}3.14 \% \\
(2)\end{array}$ & $\begin{array}{c}3.10 \% \\
\text { (1) }\end{array}$ \\
\hline
\end{tabular}

Table 8: Sobol indices for the simulation code $\Lambda=\phi(\boldsymbol{D})$.

\section{Conclusion}

This paper describes a global data-driven ROSA procedure which is able to quantify the amount of epistemic uncertainty conveyed on the failure probability $\hat{P}_{f}$ by the different parts of a copula-based distribution inferred from the available data (margins and copula). The situation corresponding to exact failure calculation and estimation via simulation-based algorithms are addressed. The computed global ROSA indices take into account the combined effect of both data scarcity and model uncertainty and allow to identify which element of the joint distribution (margins or copula) have to be refined in order to decrease the uncertainty affecting reliability assessment. The efficiency of the method is illustrated on analytical test cases and a real-world case study relative to the buckling of laminar plate. Straightforward further works will consist in coupling the proposed approach with an adaptive strategy relying on the refinement of a small number of margins to reduce uncertainty in reliability assessment. Furthermore, when the copula is identified as the most influential entity, one may wonder how to improve the state of knowledge about the dependence structure without having to collect joint observations. A technique developed by the same authors in [149] consists in looking for the pair-copula that most impacts reliability assessment. However, this ROSA algorithm rests on the assumption that all marginal distributions are prescribed. As this does not hold in the present context, some work has to be done before achieving a synergetic combination of both ROSA procedures.

\section{Acknowledgment}

The first author is currently enrolled in a PhD program co-funded by ONERA and the Occitanie region. This financial support is gratefully acknowledged. The authors would also particularly like to thank François-Xavier Irisarri and Antoine Hurmane from ONERA's Materials and Structures Department (DMAS) for proposing the real-data study of Section 5.

\section{References}

[1] J. Bucklew, Introduction to rare event simulation, Springer Science \& Business Media, 2013. 
[2] J. Morio, M. Balesdent, Estimation of rare event probabilities in complex aerospace and other systems: a practical approach, Woodhead Publishing, 2015.

[3] X.-S. Tang, D.-Q. Li, G. Rong, K.-K. Phoon, C.-B. Zhou, Impact of copula selection on geotechnical reliability under incomplete probability information, Computers and Geotechnics 49 (2013) 264-278.

[4] X.-S. Tang, D.-Q. Li, C.-B. Zhou, L.-M. Zhang, Bivariate distribution models using copulas for reliability analysis, Proceedings of the Institution of Mechanical Engineers, Part O: Journal of Risk and Reliability 227 (5) (2013) 499-512.

[5] M. Balesdent, J. Morio, L. Brevault, Rare event probability estimation in the presence of epistemic uncertainty on input probability distribution parameters, Methodology and Computing in Applied Probability 18 (1) (2016) 197-216.

[6] A. P. Dempster, Upper and lower probability inferences based on a sample from a finite univariate population, Biometrika 54 (3-4) (1967) 515-528.

[7] G. Shafer, A mathematical theory of evidence, Vol. 42, Princeton university press, 1976.

[8] G. De Cooman, D. Ruan, E. Kerre, Foundations and applications of possibility theory, in: Proceedings of FAPT 95, World Scientific, Singapore, 328 biz, 1995.

[9] D. Dubois, H. Prade, Possibility theory: an approach to computerized processing of uncertainty, Springer Science \& Business Media, 2012.

[10] S. Ferson, L. R. Ginzburg, Different methods are needed to propagate ignorance and variability, Reliability Engineering \& System Safety 54 (2-3) (1996) 133-144.

[11] S. Ferson, J. G. Hajagos, Arithmetic with uncertain numbers: rigorous and (often) best possible answers, Reliability Engineering \& System Safety 85 (1-3) (2004) 135-152.

[12] A. Gelman, et al., Prior distributions for variance parameters in hierarchical models (comment on article by browne and draper), Bayesian analysis 1 (3) (2006) 515-534.

[13] A. Gelman, J. B. Carlin, H. S. Stern, D. B. Dunson, A. Vehtari, D. B. Rubin, Bayesian data analysis, CRC press, 2013.

[14] F. Tonon, Using random set theory to propagate epistemic uncertainty through a mechanical system, Reliability Engineering \& System Safety 85 (1-3) (2004) 169-181.

[15] D. A. Alvarez, On the calculation of the bounds of probability of events using infinite random sets, International journal of approximate reasoning 43 (3) (2006) 241-267. 
[16] L. A. Zadeh, Fuzzy sets, Information and control 8 (3) (1965) 338-353.

[17] D. Dubois, H. Prade, Fundamentals of fuzzy sets, Vol. 7, Springer Science \& Business Media, 2012.

[18] Z. Wang, G. J. Klir, Fuzzy measure theory, Springer Science \& Business Media, 2013.

[19] J. Morio, M. Balesdent, D. Jacquemart, C. Vergé, A survey of rare event simulation methods for static input-output models, Simulation Modelling Practice and Theory 49 (2014) 287-304.

[20] E. F. Halpern, M. C. Weinstein, M. G. Hunink, G. S. Gazelle, Representing both first-and secondorder uncertainties by monte carlo simulation for groups of patients, Medical Decision Making 20 (3) (2000) 314-322.

[21] D. A. Alvarez, J. E. Hurtado, An efficient method for the estimation of structural reliability intervals with random sets, dependence modeling and uncertain inputs, Computers \& Structures 142 (2014) 54-63.

[22] Z. Zhang, C. Jiang, G. Wang, X. Han, First and second order approximate reliability analysis methods using evidence theory, Reliability Engineering \& System Safety 137 (2015) 40-49.

[23] M. de Angelis, E. Patelli, M. Beer, Advanced line sampling for efficient robust reliability analysis, Structural safety 52 (2015) 170-182.

[24] R. Schöbi, B. Sudret, Structural reliability analysis for p-boxes using multi-level meta-models, Probabilistic Engineering Mechanics 48 (2017) 27-38.

[25] J. C. Helton, J. D. Johnson, W. Oberkampf, C. J. Sallaberry, Sensitivity analysis in conjunction with evidence theory representations of epistemic uncertainty, Reliability Engineering \& System Safety 91 (10-11) (2006) 1414-1434.

[26] S. Sankararaman, S. Mahadevan, Separating the contributions of variability and parameter uncertainty in probability distributions, Reliability Engineering \& System Safety 112 (2013) 187-199.

[27] M. Oberguggenberger, J. King, B. Schmelzer, Classical and imprecise probability methods for sensitivity analysis in engineering: A case study, International Journal of Approximate Reasoning 50 (4) (2009) 680-693.

[28] R. Schöbi, B. Sudret, Global sensitivity analysis in the context of imprecise probabilities (pboxes) using sparse polynomial chaos expansions, Reliability Engineering \& System Safety 187 (2019) 129-141. 
[29] J.-M. Bourinet, Reliability analysis and optimal design under uncertainty-focus on adaptive surrogate-based approaches, Habilitation à diriger des recherches (HDR), Université Clermont Auvergne (2018).

[30] V. Chabridon, Reliability-oriented sensitivity analysis under probabilistic model uncertaintyapplication to aerospace systems, Ph.D. thesis (2018).

[31] L. Wang, Y. Ma, J. Zhang, X. Zhang, Y. Liu, Uncertainty quantification and structural reliability estimation considering inspection data scarcity, ASCE-ASME Journal of Risk and Uncertainty in Engineering Systems, Part A: Civil Engineering 1 (2) (2015) 04015004.

[32] J. Zhang, M. D. Shields, On the quantification and efficient propagation of imprecise probabilities resulting from small datasets, Mechanical Systems and Signal Processing 98 (2018) 465-483.

[33] J. Zhang, M. D. Shields, The effect of prior probabilities on quantification and propagation of imprecise probabilities resulting from small datasets, Computer Methods in Applied Mechanics and Engineering 334 (2018) 483-506.

[34] J. Morio, Influence of input pdf parameters of a model on a failure probability estimation, Simulation Modelling Practice and Theory 19 (10) (2011) 2244-2255.

[35] E. Veach, L. J. Guibas, Optimally combining sampling techniques for monte carlo rendering, in: Proceedings of the 22nd annual conference on Computer graphics and interactive techniques, 1995, pp. 419-428.

[36] D. P. Thunnissen, Propagating and mitigating uncertainty in the design of complex multidisciplinary systems, Ph.D. thesis, California Institute of Technology (2005).

[37] A. Der Kiureghian, O. Ditlevsen, Aleatory or epistemic? does it matter?, Structural safety 31 (2) (2009) 105-112.

[38] G. Apostolakis, The concept of probability in safety assessments of technological systems, Science 250 (4986) (1990) 1359-1364.

[39] G. Salvadori, C. De Michele, On the use of copulas in hydrology: theory and practice, Journal of Hydrologic Engineering 12 (4) (2007) 369-380.

[40] J.-T. Shiau, S. Feng, S. Nadarajah, Assessment of hydrological droughts for the yellow river, china, using copulas, Hydrological Processes: An International Journal 21 (16) (2007) 2157 2163. 
[41] X. Z. Wu, Modelling dependence structures of soil shear strength data with bivariate copulas and applications to geotechnical reliability analysis, Soils and Foundations 55 (5) (2015) 1243-1258.

[42] T.-J. Lü, X.-S. Tang, D.-Q. Li, X.-H. Qi, Modeling multivariate distribution of multiple soil parameters using vine copula model, Computers and Geotechnics 118 (2020) 103340.

[43] U. Cherubini, E. Luciano, W. Vecchiato, Copula methods in finance, John Wiley \& Sons, 2004.

[44] C. Genest, M. Gendron, M. Bourdeau-Brien, The advent of copulas in finance, in: Copulae and multivariate probability distributions in finance, Routledge, 2013, pp. 13-22.

[45] C. Czado, R. Kastenmeier, E. C. Brechmann, A. Min, A mixed copula model for insurance claims and claim sizes, Scandinavian Actuarial Journal 2012 (4) (2012) 278-305.

[46] Y. Zhang, V. Dukic, Predicting multivariate insurance loss payments under the bayesian copula framework, Journal of Risk and Insurance 80 (4) (2013) 891-919.

[47] M. Sklar, Fonctions de répartition à $n$ dimensions et leurs marges, Publ. inst. statist. univ. Paris 8 (1959) 229-231.

[48] R. B. Nelsen, An introduction to copulas, Springer Science \& Business Media, 2007.

[49] H. Joe, Dependence modeling with copulas, CRC press, 2014.

[50] F. O. Hoffman, J. S. Hammonds, Propagation of uncertainty in risk assessments: the need to distinguish between uncertainty due to lack of knowledge and uncertainty due to variability, Risk analysis 14 (5) (1994) 707-712.

[51] J. C. Helton, Treatment of uncertainty in performance assessments for complex systems, Risk analysis 14 (4) (1994) 483-511.

[52] C. Robert, The Bayesian choice: from decision-theoretic foundations to computational implementation, Springer Science \& Business Media, 2007.

[53] J. Zhang, L. Zhang, W. H. Tang, Bayesian framework for characterizing geotechnical model uncertainty, Journal of Geotechnical and Geoenvironmental Engineering 135 (7) (2009) 932-940.

[54] C. Chiu, W. Yan, K.-V. Yuen, Reliability analysis of soil-water characteristics curve and its application to slope stability analysis, Engineering Geology 135 (2012) 83-91.

[55] A. D. Kiureghian, Measures of structural safety under imperfect states of knowledge, Journal of Structural Engineering 115 (5) (1989) 1119-1140. 
[56] V. Chabridon, M. Balesdent, J.-M. Bourinet, J. Morio, N. Gayton, Evaluation of failure probability under parameter epistemic uncertainty: application to aerospace system reliability assessment, Aerospace Science and Technology 69 (2017) 526-537.

[57] H. P. Hong, Evaluation of the probability of failure with uncertain distribution parameters, Civil Engineering Systems 13 (2) (1996) 157-168.

[58] M. Pendola, Fiabilité des structures en contexte d'incertitudes statistiques et d'écarts de modélisation, Ph.D. thesis, Clermont-Ferrand 2 (2000).

[59] M. Fréchet, Sur les tableaux de corrélation dont les marges sont données, Ann. Univ. Lyon, $3^{\mathrm{e}}$ série, Sciences, Sect. A 14 (1951) 53-77.

[60] D.-Q. Li, X.-S. Tang, K.-K. Phoon, Y.-F. Chen, C.-B. Zhou, Bivariate simulation using copula and its application to probabilistic pile settlement analysis, International Journal for Numerical and Analytical Methods in Geomechanics 37 (6) (2013) 597-617.

[61] S. Ghosh, Modelling bivariate rainfall distribution and generating bivariate correlated rainfall data in neighbouring meteorological subdivisions using copula, Hydrological Processes 24 (24) (2010) 3558-3567.

[62] C. Genest, L.-P. Rivest, Statistical inference procedures for bivariate archimedean copulas, Journal of the American statistical Association 88 (423) (1993) 1034-1043.

[63] J.-D. Fermanian, et al., Goodness-of-fit tests for copulas, Journal of multivariate analysis 95 (1) (2005) 119-152.

[64] C. Genest, B. Rémillard, D. Beaudoin, Goodness-of-fit tests for copulas: A review and a power study, Insurance: Mathematics and economics 44 (2) (2009) 199-213.

[65] D. Huard, G. Évin, A.-C. Favre, Bayesian copula selection, Computational Statistics \& Data Analysis 51 (2) (2006) 809-822.

[66] D. L. Kelly, Using copulas to model dependence in simulation risk assessment, in: ASME 2007 International Mechanical Engineering Congress and Exposition, American Society of Mechanical Engineers Digital Collection, 2007, pp. 81-89.

[67] C. Czado, Analyzing dependent data with vine copulas, Lecture Notes in Statistics, Springer.

[68] O. Okhrin, Y. Okhrin, W. Schmid, Properties of hierarchical archimedean copulas, Statistics \& Risk Modeling with Applications in Finance and Insurance 30 (1) (2013) 21-54. 
[69] G. Elidan, Copula bayesian networks, in: Advances in neural information processing systems, 2010, pp. 559-567.

[70] P. Krupskii, H. Joe, Factor copula models for multivariate data, Journal of Multivariate Analysis 120 (2013) 85-101.

[71] G. Mazo, S. Girard, F. Forbes, A class of multivariate copulas based on products of bivariate copulas, Journal of Multivariate Analysis 140 (2015) 363-376.

[72] S. Kotz, N. Balakrishnan, N. L. Johnson, Continuous multivariate distributions, Volume 1: Models and applications, John Wiley \& Sons, 2004.

[73] F. Faridafshin, A. Naess, Multivariate log-concave probability density class for structural reliability applications, Structural Safety 69 (2017) 57-67.

[74] F. Wang, H. Li, Towards reliability evaluation involving correlated multivariates under incomplete probability information: A reconstructed joint probability distribution for isoprobabilistic transformation, Structural Safety 69 (2017) 1-10.

[75] N. Benoumechiara, B. Michel, P. Saint-Pierre, N. Bousquet, Detecting and modeling worstcase dependence structures between random inputs of computational reliability models, arXiv preprint arXiv:1804.10527.

[76] I. Montes, E. Miranda, R. Pelessoni, P. Vicig, Sklar's theorem in an imprecise setting, Fuzzy Sets and Systems 278 (2015) 48-66.

[77] R. Pelessoni, P. Vicig, M. Ignacio, M. Enrique, Imprecise copulas and bivariate stochastic orders.

[78] T. Coolen-Maturi, F. P. Coolen, N. Muhammad, Predictive inference for bivariate data: Combining nonparametric predictive inference for marginals with an estimated copula, Journal of Statistical Theory and Practice 10 (3) (2016) 515-538.

[79] R. Schefzik, T. L. Thorarinsdottir, T. Gneiting, et al., Uncertainty quantification in complex simulation models using ensemble copula coupling, Statistical science 28 (4) (2013) 616-640.

[80] J. Zhang, M. Shields, On the quantification and efficient propagation of imprecise probabilities with copula dependence, International Journal of Approximate Reasoning.

[81] A. Saltelli, M. Ratto, T. Andres, F. Campolongo, J. Cariboni, D. Gatelli, M. Saisana, S. Tarantola, Global sensitivity analysis: the primer, John Wiley \& Sons, 2008.

[82] S. Rahman, Stochastic sensitivity analysis by dimensional decomposition and score functions, Probabilistic Engineering Mechanics 24 (3) (2009) 278-287. 
[83] H. Millwater, Universal properties of kernel functions for probabilistic sensitivity analysis, Probabilistic Engineering Mechanics 24 (1) (2009) 89-99.

[84] I. Lee, K. K. Choi, Y. Noh, L. Zhao, D. Gorsich, Sampling-based stochastic sensitivity analysis using score functions for rbdo problems with correlated random variables, Journal of Mechanical Design $133(2)$.

[85] P. Wang, Z. Lu, K. Zhang, S. Xiao, Z. Yue, Copula-based decomposition approach for the derivative-based sensitivity of variance contributions with dependent variables, Reliability Engineering \& System Safety 169 (2018) 437-450.

[86] Y.-T. Wu, Adaptive importance sampling (ais)-based system reliability sensitivity analysis method, in: Probabilistic Structural Mechanics: Advances in Structural Reliability Methods, Springer, 1994, pp. 550-564.

[87] S. Song, Z. Lu, H. Qiao, Subset simulation for structural reliability sensitivity analysis, Reliability Engineering \& System Safety 94 (2) (2009) 658-665.

[88] P. Wang, Z. Lu, Z. Tang, A derivative based sensitivity measure of failure probability in the presence of epistemic and aleatory uncertainties, Computers \& Mathematics with Applications 65 (1) (2013) 89-101.

[89] I. Sobol, A. Gresham, On an alternative global sensitivity estimators, Proceedings of SAMO (1995) 40-42.

[90] S. Kucherenko, et al., Derivative based global sensitivity measures and their link with global sensitivity indices, Mathematics and Computers in Simulation 79 (10) (2009) 3009-3017.

[91] I. M. Sobol, Sensitivity analysis for non-linear mathematical models, Mathematical modelling and computational experiment 1 (1993) 407-414.

[92] H. Rabitz, Ö. F. Aliş, J. Shorter, K. Shim, Efficient input-output model representations, Computer physics communications 117 (1-2) (1999) 11-20.

[93] I. M. Sobol, Global sensitivity indices for nonlinear mathematical models and their monte carlo estimates, Mathematics and computers in simulation 55 (1-3) (2001) 271-280.

[94] A. Saltelli, Making best use of model evaluations to compute sensitivity indices, Computer physics communications 145 (2) (2002) 280-297.

[95] P. Wei, J. Song, S. Bi, M. Broggi, M. Beer, Z. Lu, Z. Yue, Non-intrusive stochastic analysis with parameterized imprecise probability models: 1. performance estimation, Mechanical Systems and Signal Processing 124 (2019) 349-368. 
[96] P. Wei, J. Song, S. Bi, M. Broggi, M. Beer, Z. Lu, Z. Yue, Non-intrusive stochastic analysis with parameterized imprecise probability models: 2. reliability and rare events analysis, Mechanical Systems and Signal Processing 126 (2019) 227-247.

[97] J. Song, P. Wei, M. Valdebenito, S. Bi, M. Broggi, M. Beer, Z. Lei, Generalization of nonintrusive imprecise stochastic simulation for mixed uncertain variables, Mechanical Systems and Signal Processing 134 (2019) 106316.

[98] V. Žanić, K. Žiha, Sensivity to correlation in structural reliability problems, Transaction of FAMENA 25 (2) (2001) 1.

[99] J.-M. Bourinet, Form sensitivities to distribution parameters with the nataf transformation, in: Risk and Reliability Analysis: Theory and Applications, Springer, 2017, pp. 277-302.

[100] M. Beer, Y. Zhang, S. T. Quek, K. K. Phoon, Reliability analysis with scarce information: Comparing alternative approaches in a geotechnical engineering context, Structural Safety 41 (2013) 1-10.

[101] A. G. Awadallah, H. Saad, A. Elmoustafa, A. Hassan, Reliability assessment of water structures subject to data scarcity using the scs-cn model, Hydrological Sciences Journal 61 (4) (2016) $696-710$.

[102] C. W. Zhang, R. Pan, T. N. Goh, Reliability assessment of high-quality new products with data scarcity, International Journal of Production Research (2020) 1-13.

[103] S. Sankararaman, Uncertainty quantification and integration in engineering systems, Vanderbilt University, 2012.

[104] H. Akaike, A new look at the statistical model identification, IEEE transactions on automatic control 19 (6) (1974) 716-723.

[105] V. I. Bogachev, Measure theory, Vol. 1, Springer Science \& Business Media, 2007.

[106] B. Iooss, P. Lemaître, A review on global sensitivity analysis methods, in: Uncertainty management in simulation-optimization of complex systems, Springer, 2015, pp. 101-122.

[107] F. Gamboa, A. Janon, T. Klein, A. Lagnoux, et al., Sensitivity analysis for multidimensional and functional outputs, Electronic Journal of Statistics 8 (1) (2014) 575-603.

[108] F. Gamboa, T. Klein, A. Lagnoux, L. Moreno, Sensitivity analysis in general metric spaces, arXiv preprint arXiv:2002.04465. 
[109] A. J. McNeil, R. Frey, P. Embrechts, Quantitative risk management: concepts, techniques and tools-revised edition, Princeton university press, 2015.

[110] B. Choroś, R. Ibragimov, E. Permiakova, Copula estimation, in: Copula theory and its applications, Springer, 2010, pp. 77-91.

[111] H. Joe, Multivariate models and multivariate dependence concepts, CRC Press, 1997.

[112] P. Silvapulle, G. Kim, M. J. Silvapulle, et al., Robustness of a semiparametric estimator of a copula, in: Econometric Society 2004 Australasian Meetings, no. 317, Econometric Society, 2004.

[113] C. Genest, K. Ghoudi, L.-P. Rivest, A semiparametric estimation procedure of dependence parameters in multivariate families of distributions, Biometrika 82 (3) (1995) 543-552.

[114] J. H. Shih, T. A. Louis, Inferences on the association parameter in copula models for bivariate survival data, Biometrics (1995) 1384-1399.

[115] G. Kim, M. J. Silvapulle, P. Silvapulle, Comparison of semiparametric and parametric methods for estimating copulas, Computational Statistics \& Data Analysis 51 (6) (2007) 2836-2850.

[116] B. Efron, Bootstrap methods: another look at the jackknife, in: Breakthroughs in statistics, Springer, 1992, pp. 569-593.

[117] R. J. Tibshirani, B. Efron, An introduction to the bootstrap, Monographs on statistics and applied probability 57 (1993) 1-436.

[118] P. I. Good, Resampling methods, Springer, 2006.

[119] J. Shao, D. Tu, The jackknife and bootstrap, Springer Science \& Business Media, 2012.

[120] G. Archer, A. Saltelli, I. Sobol, Sensitivity measures, anova-like techniques and the use of bootstrap, Journal of Statistical Computation and Simulation 58 (2) (1997) 99-120.

[121] D. C. Montgomery, E. A. Peck, G. G. Vining, Introduction to linear regression analysis, Vol. 821, John Wiley \& Sons, 2012.

[122] W. Fellin, J. King, A. Kirsch, M. Oberguggenberger, Uncertainty modelling and sensitivity analysis of tunnel face stability, Structural Safety 32 (6) (2010) 402-410.

[123] F. Gamboa, A. Janon, T. Klein, A. Lagnoux, C. Prieur, Statistical inference for sobol pick-freeze monte carlo method, Statistics 50 (4) (2016) 881-902.

[124] O. Ditlevsen, H. O. Madsen, Structural reliability methods, Vol. 178, Wiley New York, 1996. 
[125] M. Lemaire, Structural reliability, John Wiley \& Sons, 2013.

[126] H. Kahn, T. E. Harris, Estimation of particle transmission by random sampling, National Bureau of Standards applied mathematics series 12 (1951) 27-30.

[127] M. Shinozuka, Basic analysis of structural safety, Journal of Structural Engineering 109 (3) (1983) $721-740$.

[128] A. Harbitz, An efficient sampling method for probability of failure calculation, Structural safety 3 (2) (1986) 109-115.

[129] D. Siegmund, Importance sampling in the monte carlo study of sequential tests, The Annals of Statistics (1976) 673-684.

[130] J. S. Stadler, S. Roy, Adaptive importance sampling (digital communication), IEEE journal on selected areas in communications 11 (3) (1993) 309-316.

[131] M.-S. Oh, J. O. Berger, Adaptive importance sampling in monte carlo integration, Journal of Statistical Computation and Simulation 41 (3-4) (1992) 143-168.

[132] R. Y. Rubinstein, D. P. Kroese, The cross-entropy method: a unified approach to combinatorial optimization, Monte-Carlo simulation and machine learning, Springer Science \& Business Media, 2013.

[133] G. H. Givens, A. E. Raftery, Local adaptive importance sampling for multivariate densities with strong nonlinear relationships, Journal of the American Statistical Association 91 (433) (1996) $132-141$.

[134] J. C. Neddermeyer, Computationally efficient nonparametric importance sampling, Journal of the American Statistical Association 104 (486) (2009) 788-802.

[135] Y. B. Kim, D. S. Roh, M. Y. Lee, Nonparametric adaptive importance sampling for rare event simulation, in: 2000 Winter Simulation Conference Proceedings (Cat. No. 00CH37165), Vol. 1, IEEE, 2000, pp. 767-772.

[136] P. Zhang, Nonparametric importance sampling, Journal of the American Statistical Association 91 (435) (1996) 1245-1253.

[137] J. Morio, Extreme quantile estimation with nonparametric adaptive importance sampling, Simulation Modelling Practice and Theory 27 (2012) 76-89.

[138] R. J. Beckman, M. D. McKay, Monte carlo estimation under different distributions using the same simulation, Technometrics 29 (2) (1987) 153-160. 
[139] M. I. Reiman, A. Weiss, Sensitivity analysis for simulations via likelihood ratios, Operations Research 37 (5) (1989) 830-844.

[140] E. Veach, L. Guibas, Bidirectional estimators for light transport, in: Photorealistic Rendering Techniques, Springer, 1995, pp. 145-167.

[141] E. Veach, Robust Monte Carlo methods for light transport simulation, Vol. 1610, Stanford University PhD thesis, 1997.

[142] B. W. Silverman, Density estimation for statistics and data analysis, Vol. 26, CRC press, 1986.

[143] M. R. Eslami, J. Eslami, Jacobs, Buckling and postbuckling of beams, plates, and shells, Springer, 2018.

[144] L. C. Bank, J. Yin, Buckling of orthotropic plates with free and rotationally restrained unloaded edges, Thin-Walled Structures 24 (1) (1996) 83-96.

[145] C. Bouvet, Mechanics of Aeronautical Composite Materials, John Wiley \& Sons, 2017.

[146] D. Kurowicka, R. M. Cooke, Uncertainty analysis with high dimensional dependence modelling, John Wiley \& Sons, 2006.

[147] E. Torre, S. Marelli, P. Embrechts, B. Sudret, A general framework for data-driven uncertainty quantification under complex input dependencies using vine copulas, Probabilistic Engineering Mechanics 55 (2019) 1-16.

[148] C. Jiang, W. Zhang, X. Han, B. Ni, L. Song, A vine-copula-based reliability analysis method for structures with multidimensional correlation, Journal of Mechanical Design 137 (6).

[149] G. Sarazin, J. Morio, A. Lagnoux, M. Balesdent, L. Brevault, Sensitivity analysis of risk assessment with data-driven dependence modeling, in: 29th European Safety and Reliability Conference, 2019.

[150] A. Harbitz, Efficient and accurate probability of failure calculation by the use of importance sampling technique, in: Proc. of ICASP, Vol. 4, 1983, pp. 825-836.

[151] R. Melchers, Importance sampling in structural systems, Structural safety 6 (1) (1989) 3-10. 


\section{Appendix A. Adaptation of the resampling strategy to enable data assimilation}

Appendix A.1. Pseudo-code

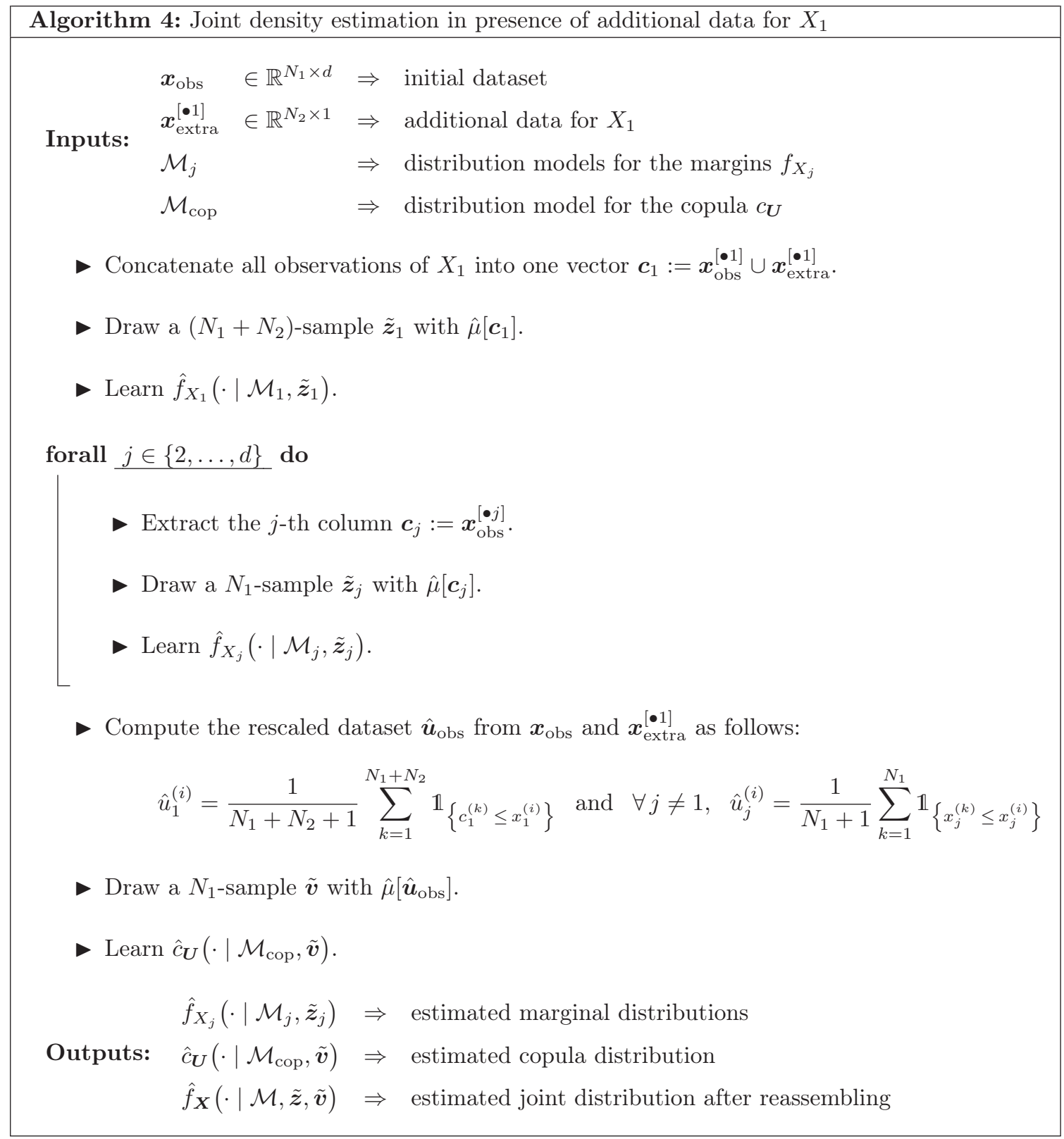

\section{Appendix A.2. Matrix manipulations}

The resampling mechanism used to assimilate new observations of $X_{1}$ is denoted by $\mathcal{B}_{1}$ and obeys the following scheme :

$$
\begin{aligned}
& \mathcal{B}_{1}: \mathbb{R}^{N_{1} \times d} \times \mathbb{R}^{N_{2}} \longrightarrow \mathbb{R}^{N_{1}+N_{2}} \times \mathbb{R}^{N} \times \ldots \times \mathbb{R}^{N} \times[0,1]^{N} \\
& \begin{array}{lllll}
\boldsymbol{x}_{\mathrm{obs}} & \boldsymbol{x}_{\text {extra }}^{[\bullet 1]} \longmapsto \quad \tilde{\boldsymbol{z}}_{1} & \tilde{z}_{2}, \ldots, \tilde{\boldsymbol{z}}_{d} & \tilde{\boldsymbol{v}}
\end{array}
\end{aligned}
$$




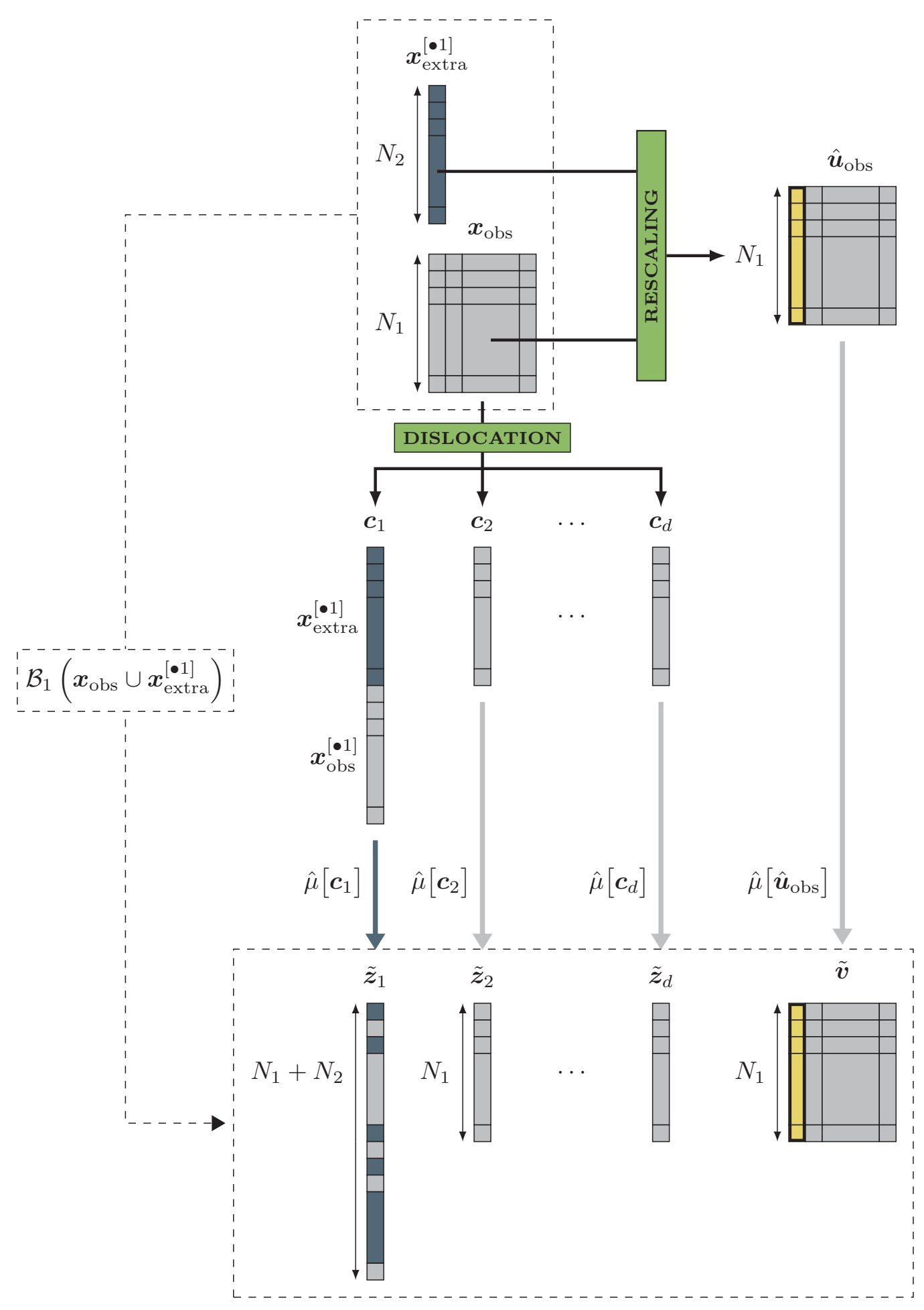

Figure A.12: Resampling mechanism $\mathcal{B}_{1}$ adapted from $\mathcal{B}$ in order to assimilate additional observations of $X_{1}$ 


\section{Appendix B. Gaussian Importance Sampling (GIS)}

In Section 4.4, the performance function $\phi(\cdot)$ is given by:

$$
Y=\phi(\boldsymbol{X})=a_{1} X_{1}+a_{2} X_{2}+a_{3} X_{3}+a_{4} X_{1} X_{2}
$$

and any estimated input PDF may be denoted by $\hat{f}_{\boldsymbol{X}}(\cdot \mid \tilde{\boldsymbol{z}}, \hat{\boldsymbol{h}}, \hat{\boldsymbol{\rho}})$ since it is reassembled from:

$$
\hat{f}_{X_{j}}\left(\cdot \mid \mathcal{M}_{j}, \tilde{\boldsymbol{z}}_{j}\right)=\hat{f}_{\mathrm{KDE}}\left(\cdot \mid \hat{h}_{j}, \tilde{\boldsymbol{z}}_{j}\right) \quad \text { and } \quad \hat{c}_{\boldsymbol{U}}\left(\cdot \mid \mathcal{M}_{\mathrm{cop}}, \tilde{\boldsymbol{v}}\right)=\hat{c}_{\mathcal{N}}(\cdot \mid \hat{\boldsymbol{\rho}})
$$

The failure probability associated to the input $\operatorname{PDF} \hat{f}_{\boldsymbol{X}}(\cdot \mid \tilde{\boldsymbol{z}}, \hat{\boldsymbol{h}}, \hat{\boldsymbol{\rho}})$ can be expressed as follows :

$$
P_{f}(\tilde{\boldsymbol{z}}, \hat{\boldsymbol{h}}, \hat{\boldsymbol{\rho}})=\int_{\mathbb{R}^{3}} \mathbb{1}_{D_{f}}(\boldsymbol{x}) \hat{f}_{\boldsymbol{X}}(\boldsymbol{x} \mid \tilde{\boldsymbol{z}}, \hat{\boldsymbol{h}}, \hat{\boldsymbol{\rho}}) \mathrm{d} \boldsymbol{x}
$$

Let us imagine that one tries to estimate $P_{f}(\tilde{\boldsymbol{z}}, \hat{\boldsymbol{h}}, \hat{\boldsymbol{\rho}})$ with the CMC approach. This situation is represented on Figure B.13a where no input particle hits the failure domain $\hat{D}_{f}^{\mathcal{N}}$ defined in Eq. (83). A better strategy could be to sample in the vicinity of the MPFP. To achieve so, one option might be to consider a Gaussian auxiliary distribution $\mathcal{N}\left(\hat{\boldsymbol{x}}^{\star}, \boldsymbol{\Sigma}\right)$. Gaussian importance sampling (GIS) is deeply investigated in $[150,151]$ where it is advised to take a diagonal covariance matrix $\boldsymbol{\Sigma}$ where the $j$-th coefficient is roughly equal to $\mathbb{V}\left(X_{j}\right)$. In the context of Section 4.4, this yields to $\boldsymbol{I}_{3}$. In what follows, it is accepted that using $\boldsymbol{I}_{3}$ as covariance matrix for the auxiliary PDF is an appropriate tuning. The resulting GIS estimator is therefore provided by :

$$
\hat{P}_{f}(\tilde{\boldsymbol{z}}, \hat{\boldsymbol{h}}, \hat{\boldsymbol{\rho}})=\frac{1}{N_{s}} \sum_{i=1}^{N_{s}} \mathbb{1}_{\left\{Y^{(i)}>T\right\}} \frac{\hat{f}_{\boldsymbol{X}}\left(\boldsymbol{X}^{(i)} \mid \tilde{\boldsymbol{z}}, \hat{\boldsymbol{h}}, \hat{\boldsymbol{\rho}}\right)}{\varphi_{3}\left(\boldsymbol{X}^{(i)}-\hat{\boldsymbol{x}}^{\star}\right)} \quad \text { with }\left\{\begin{array}{l}
\boldsymbol{X}^{(i)} \stackrel{\text { i.i.d }}{\sim} \mathcal{N}\left(\boldsymbol{x}^{\star}, \boldsymbol{I}_{3}\right) \\
Y^{(i)}=\phi\left(\boldsymbol{X}^{(i)}\right)
\end{array}\right.
$$

The reader is invited to have a look at Figure B.13b in order to intuitively understand why this change of sampling distribution is actually effective. GIS is thus a two-step procedure including:

1. The computation of $\hat{\boldsymbol{x}}^{\star}$ through FORM by means of $N^{\star}$ black-box calls $\left(N^{\star} \ll N_{s}\right)$.

2. The implementation of an IS methodology based on the auxiliary PDF $\boldsymbol{x} \longmapsto \varphi_{3}\left(\boldsymbol{x}-\hat{\boldsymbol{x}}^{\star}\right)$.

At first sight, the GIS approach may seem very crude in order to construct a suboptimal auxiliary distribution. It is indeed the case in general. However, it is quite enough for the very elementary test case examined here. It is assumed that $a_{1}, \ldots, a_{4}$ are positive and the following notations are established:

$$
\gamma_{\boldsymbol{a}}=-\frac{a_{2}}{a_{4}} \quad ; \quad \delta_{\boldsymbol{a}}=-\frac{a_{1}}{a_{4}} \quad ; \quad \xi_{\boldsymbol{a}}=\frac{T+a_{2} a_{4}}{a_{3} a_{4}} \quad ; \quad \kappa_{\boldsymbol{a}}\left(x_{1}, x_{3}\right)=\frac{T-a_{1} x_{1}-a_{3} x_{3}}{a_{2}+a_{4} x_{1}}
$$




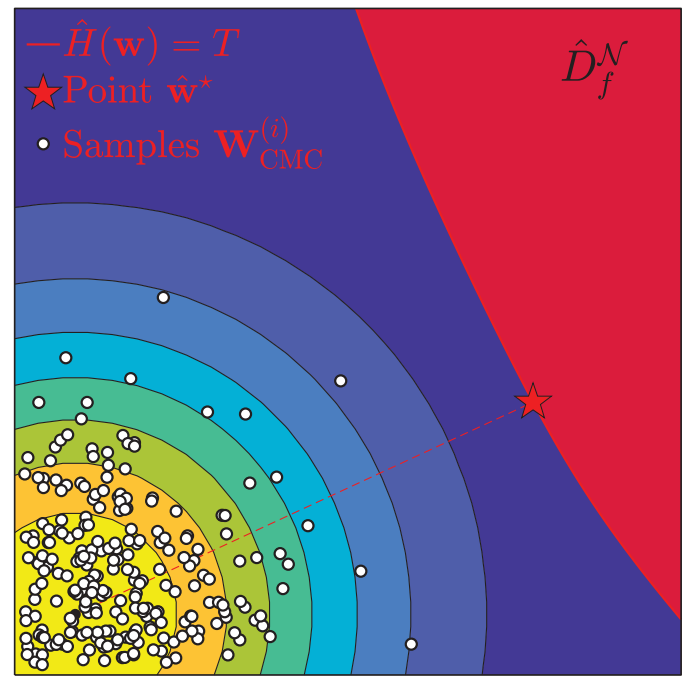

(a) Crude Monte Carlo (CMC)

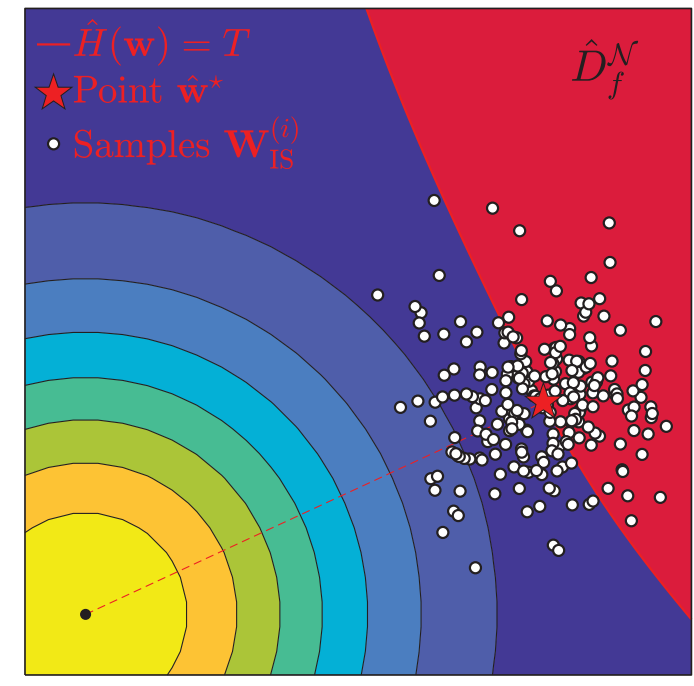

(b) Gaussian Importance Sampling (GIS)

Figure B.13: Visualization of two sampling techniques in the standard normal space

After small efforts, one can see that the failure domain $D_{f}$ (in the physical space) may be divided into four subdomains:

$$
\begin{aligned}
D_{f} & =\left\{\boldsymbol{x} \in \mathbb{R}^{d}: \phi(\boldsymbol{x})>T\right\} \\
& =\left(D_{f} \cap\left\{x_{3}<\xi_{a}\right\}\right) \cup\left(D_{f} \cap\left\{x_{3}>\xi_{a}\right\}\right), \\
& =\left(D_{f}^{A_{1}} \cup D_{f}^{A_{2}}\right) \cup\left(D_{f}^{B_{1}} \cup D_{f}^{B_{2}}\right),
\end{aligned}
$$

which are defined by:

$$
\begin{aligned}
& D_{f}^{A_{1}}:=\left\{x_{1}>\gamma_{\boldsymbol{a}} \text { avec } x_{3}<\xi_{\boldsymbol{a}} \text { et } x_{2}>\kappa_{\boldsymbol{a}}\left(x_{1}, x_{3}\right)\right\} ; \\
& D_{f}^{A_{2}}:=\left\{x_{1}<\gamma_{\boldsymbol{a}} \text { avec } x_{3}<\xi_{\boldsymbol{a}} \text { et } x_{2}<\kappa_{\boldsymbol{a}}\left(x_{1}, x_{3}\right)\right\} ; \\
& D_{f}^{B_{1}}:=\left\{x_{1}>\gamma_{\boldsymbol{a}} \text { avec } x_{3}>\xi_{\boldsymbol{a}} \text { et } x_{2}>\kappa_{\boldsymbol{a}}\left(x_{1}, x_{3}\right)\right\} ; \\
& D_{f}^{B_{2}}:=\left\{x_{1}<\gamma_{\boldsymbol{a}} \text { avec } x_{3}>\xi_{\boldsymbol{a}} \text { et } x_{2}<\kappa_{\boldsymbol{a}}\left(x_{1}, x_{3}\right)\right\} .
\end{aligned}
$$

The plane $x_{3}=\xi_{a}$ separates the two subdomains $D_{f}^{A_{1}}$ et $D_{f}^{A_{2}}$ from the two subdomains $D_{f}^{B_{1}}$ et $D_{f}^{B_{2}}$. One may refer to Figure B.14 for an overview of the possible failure modes. Figures B.14a and B.14b show how the profile of the limit state evolves on either side of the plane $x_{3}=\xi_{a}$. Unlike $D_{f}^{A_{1}}$ and $D_{f}^{A_{2}}$ that are fully disconnected, $D_{f}^{B_{1}}$ and $D_{f}^{B_{2}}$ are tangent along the plane $x_{1}=\gamma_{\boldsymbol{a}}$. For the numerical values chosen in Section 4.4, $\mu_{3}<\xi_{\boldsymbol{a}}$ and this means that $\boldsymbol{\mu}$ is located in the same half-space than the subdomains $D_{f}^{A_{1}}$ and $D_{f}^{A_{2}}$. Furthermore, the values assigned to $\mu_{1}$ and $\mu_{2}$ are such that $\boldsymbol{\mu}$ is much 


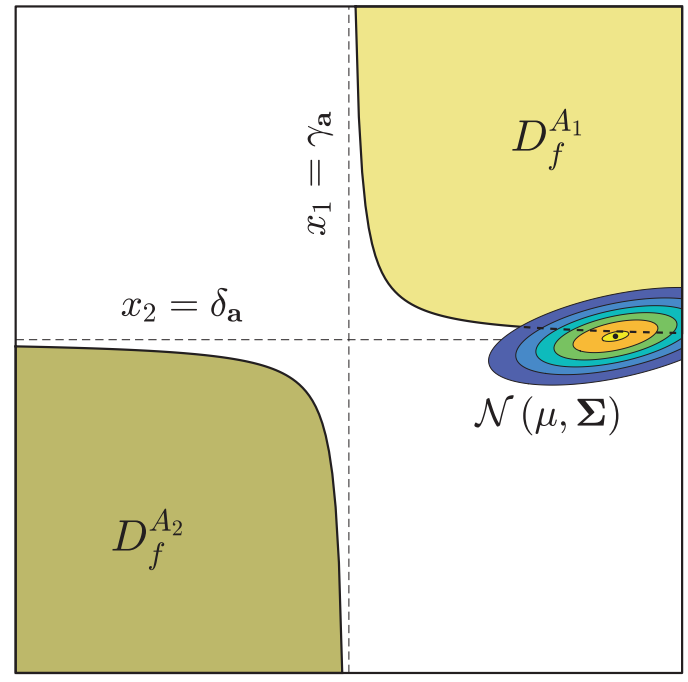

(a) Cross-section plane $x_{3}=\alpha$ with $\alpha<\xi_{a}$

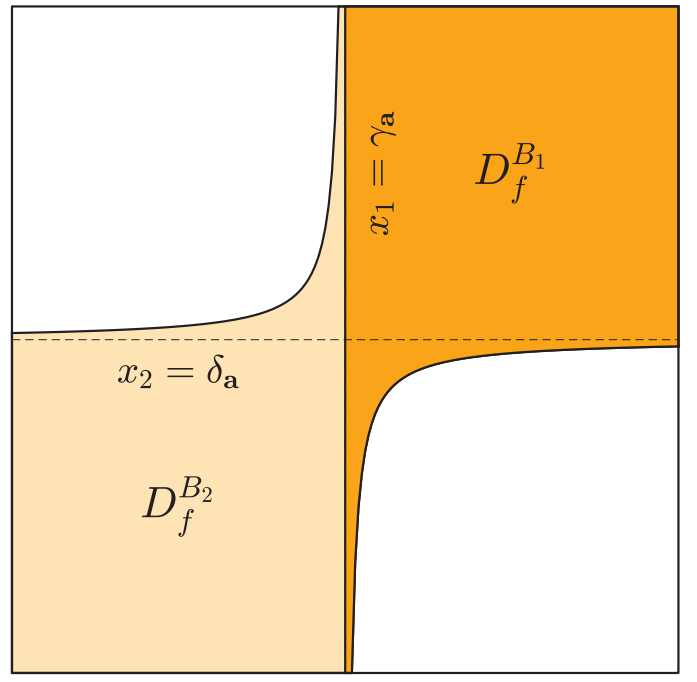

(b) Cross-section plane $x_{3}=\alpha$ with $\alpha>\xi_{a}$

Figure B.14: Visualization of the failure domain $D_{f}$ in the physical space

closer to $D_{f}^{A_{1}}$ than $D_{f}^{A_{2}}$. In view of this, it is quite reasonable to write that:

$$
P_{f}=\int_{D_{f}} f_{\boldsymbol{X}}(\boldsymbol{x}) \mathrm{d} \boldsymbol{x} \approx \int_{D_{f}^{A_{1}}} f_{\boldsymbol{X}}(\boldsymbol{x}) \mathrm{d} \boldsymbol{x}
$$

Because of its characteristics, $f_{\boldsymbol{X}}$ only activates $D_{f}^{A_{1}}$ and resorting to a unimodal auxiliary PDF is therefore sensible. 